\title{
Evidence for diffuse molecular gas and dust in the hearts of gamma-ray burst host galaxies
}

\section{Unveiling the nature of high-redshift damped Lyman- $\alpha$ systems}

\author{
J. Bolmer ${ }^{1,2}$, C. Ledoux ${ }^{1}$, P. Wiseman ${ }^{3}$, A. De $\mathrm{Cia}^{4}$, J. Selsing ${ }^{5}$, P. Schady ${ }^{2,6}$, J. Greiner $^{2}$, S. Savaglio ${ }^{7}$, \\ J. M. Burgess ${ }^{2}$, V. D’Elia ${ }^{8}$, J. P. U. Fynbo ${ }^{5}$, P. Goldoni ${ }^{9}$, D. H. Hartmann ${ }^{10}$, K. E. Heintz ${ }^{11}$, P. Jakobsson ${ }^{11}$, J. Japelj ${ }^{12}$, \\ L. Kaper ${ }^{12}$, N. R. Tanvir ${ }^{13}$, P. M. Vreeswijk ${ }^{14}$, and T. Zafar ${ }^{15}$
}

(Affiliations can be found after the references)

Received 13 October 2018 / Accepted 16 January 2019

\begin{abstract}
Context. Damped Lyman- $\alpha$ (DLA) absorption-line systems at the redshifts of gamma-ray burst (GRB) afterglows offer a unique way to probe the physical conditions within star-forming galaxies in the early Universe.

Aims. Here we built up a large sample of 22 GRBs at redshifts $z>2$ observed with VLT/X-shooter in order to determine the abundances of hydrogen, metals, dust, and molecular species. This allows us to study the metallicity and dust depletion effects in the neutral interstellar medium at high redshift and to answer the question of whether (and why) there might be a lack of $\mathrm{H}_{2}$ in GRB-DLAs.

Methods. We developed new methods based on the Bayesian inference package, PyMC, to FIT absorption lines and measure the column densities of different metal species as well as atomic and molecular hydrogen. The derived relative abundances are used to FIT dust depletion sequences and determine the dust-to-metals ratio and the host-galaxy intrinsic visual extinction. Additionally, we searched for the absorption signatures of vibrationally-excited $\mathrm{H}_{2}$ and carbon monoxide.

Results. We find that there is no lack of $\mathrm{H}_{2}$-bearing GRB-DLAs. We detect absorption lines from molecular hydrogen in 6 out of $22 \mathrm{GRB}$ afterglow spectra, with molecular fractions ranging between $f \simeq 5 \times 10^{-5}$ and $f \simeq 0.04$, and claim tentative detections in three additional cases. For the remainder of the sample, we measure, depending on $\mathrm{S} / \mathrm{N}$, spectral coverage and instrumental resolution, more or less stringent upper limits. The GRB-DLAs in our sample have on average low metallicities, $\overline{[X / H]} \approx-1.3$, comparable to the population of extremely-strong QSO-DLAs $(\log N(\mathrm{HI})>21.5)$. Furthermore, $\mathrm{H}_{2}$-bearing GRB-DLAs are found to be associated with significant dust extinction, $A_{V}>0.1 \mathrm{mag}$, and dust-to-metals ratios $\mathcal{D T M}>0.4$, confirming the importance of dust grains for the production of molecules. All these systems exhibit neutral hydrogen column densities $\log N(\mathrm{HI})>21.7$. The overall fraction of $\mathrm{H}_{2}$ detections in GRB-DLAs is $\geq 27 \%$ (41\% including tentative detections), which is three to four times larger than in the general QSO-DLA population. For $2<z<4$, and considering column densities $\log N(\mathrm{HI})>21.7$, the $\mathrm{H}_{2}$ detection fraction is $60-80 \%$ in GRB-DLAs and in extremely strong QSO-DLAs. This is likely due to the fact that both GRB- and QSO-DLAs with high neutral hydrogen column densities are probed by sight-lines with small impact parameters, indicating that the absorbing gas is associated with the inner regions of the absorbing galaxy, where the gas pressure is higher and the conversion of $\mathrm{H}$ I to $\mathrm{H}_{2}$ takes place. In the case of GRB hosts, this diffuse molecular gas is located at distances $\gtrsim 500 \mathrm{pc}$ from the GRB and hence is unrelated to the star-forming region where the event occurred.
\end{abstract}

Key words. ISM: abundances - dust, extinction - ISM: molecules - techniques: spectroscopic - galaxies: high-redshift

\section{Introduction}

Luminous background sources such as quasars (QSOs) or gamma-ray bursts (GRBs) offer a unique way to probe star-forming regions in high redshift galaxies. Usually, strong Lyman- $\alpha$ absorption features, the so-called damped Lyman- $\alpha$ absorbers (DLAs) with $\log N(\mathrm{HI})>20.3$ (Wolfe et al. 2005), are evident in the optical spectra of GRB afterglows (Fynbo et al. 2009; Tanvir et al. 2019). DLAs contain most of the neutral gas in the Universe and represent a reservoir of gas available for star formation (McKee \& Ostriker 2007; Altay et al. 2011). Spectra of GRB afterglows can thus be used to study the different phases of the gas detected in absorption, including circumgalactic and the interstellar medium (ISM), as well as dust and molecular phases (for a recent review see Schady 2017). Molecular hydrogen is of particular interest because its presence is tightly correlated with the formation of stars (Bigiel et al. 2008, 2011;
Leroy et al. 2013; Krumholz et al. 2012). However, due to the lacking dipole moment and the low mass of the molecule, it is hard to study $\mathrm{H}_{2}$ in emission at high redshift $(z \gtrsim 2)$, because the rotational transitions require high temperatures to be excited (Kennicutt \& Evans 2012). Therefore, carbon monoxide is commonly used as a tracer of molecular gas, but with the drawback that the conversion factor to $\mathrm{H}_{2}$ is still uncertain and likely also varies on different scales and for different gas properties (Tacconi et al. 2008; Bolatto et al. 2013; Gong et al. 2018). Fortunately, for DLAs at redshifts larger than $z>2$ the Lyman and Werner bands of molecular hydrogen are shifted into the observed UV band and $\mathrm{H}_{2}$ absorption lines can thus be detected in spectra of GRBs and QSOs obtained with ground-based instruments like VLT/X-shooter (Vernet et al. 2011).

The $\mathrm{H}_{2}$ content of a star-forming region is the result of the balance between its formation on dust grains and its dissociation by UV photons from the interstellar radiation field. 
For GRB-DLAs also the UV flux from the GRB itself could potentially photodissociate the molecular gas or pump it to its vibrationally excited states (Draine 2000; Draine \& Hao 2002). Additionally, large dust masses or the molecular hydrogen itself can provide (self-)shielding against the destructive UV radiation (Krumholz et al. 2009). Dust, however, also efficiently attenuates the background UV flux from the GRB, which makes it difficult to obtain high-resolution spectra of very dusty sight-lines ( $A_{V} \gtrsim 1 \mathrm{mag}$, Greiner et al. 2011), and adds to the difficulty of identifying the $\mathrm{H}_{2}$ absorption lines and distinguish them from the Lyman- $\alpha$ forest. So, while it remains difficult to study dark molecular clouds ( $A_{V}>0.5 \mathrm{mag}$ ), GRBs offer an ideal probe of the diffuse or translucent gas in high redshift galaxies.

Absorption from $\mathrm{H}_{2}$ is generally detected in $10 \%$ or less of QSO-DLA systems (Ledoux et al. 2003; Noterdaeme et al. 2008; Balashev et al. 2014; Jorgenson et al. 2014), but with a strong dependence on the neutral hydrogen column density, with the fraction increasing when selecting QSO-DLAs with large neutral hydrogen column densities (Noterdaeme et al. 2015a). Recently Balashev et al. (2017) and Ranjan et al. (2018) also reported the detection of very large $\mathrm{H}_{2}$ column densities in two other extremely strong DLAs (ESDLAs, defined as having $\log N(\mathrm{HI}) \gtrsim 21.5)$. Since GRBs are linked to star formation and originate from the inner regions of their host galaxies (Fruchter et al. 2006; Lyman et al. 2017), where they are usually behind very high column densities of gas (Jakobsson et al. 2006; Pontzen et al. 2008), one would expect to find a higher fraction of $\mathrm{H}_{2}$-bearing systems in GRB-DLAs compared to QSO-DLAs (Zwaan \& Prochaska 2006); also because the absorbing gas is located at distances from the explosion site where the influence of the ionizing UV radiation of the GRB itself should be negligible (50 to more than several hundred parsec; Prochaska et al. 2006; Vreeswijk et al. 2007, 2011; Ledoux et al. 2009; D'Elia et al. 2009; Hartoog et al. 2013). However, first searches for absorption from molecular hydrogen in small samples of GRB-DLAs were surprisingly unsuccessful. For example, from the lack of molecular hydrogen in a sample of 5 GRB afterglows, Tumlinson et al. (2007) argues that a deficiency of molecular gas in GRB-DLAs compared to QSO-DLAs, could be the result of a combination of lower metallicity and a stronger UV radiation field in GRB host galaxies. Later, from the analysis of the physical conditions in a sample of 7 GRB-DLAs, Ledoux et al. (2009) concludes that the lack of $\mathrm{H}_{2}$ can be explained by the low metallicities and depletion factors, but also by the moderate particle densities in the systems: large ( $\gtrsim 100 \mathrm{pc})$, metal-poor atomic clouds with high temperatures $(T>1000 \mathrm{~K})$. Since then, absorption from molecular hydrogen has only been identified in a handful of GRB-DLAs: GRB 080607 (Prochaska et al. 2009), 120327A (D'Elia et al. 2014), 120815A (Krühler et al. 2013), and 121024A Friis et al. (2015; possibly also in GRB 060206; Fynbo et al. 2006) ${ }^{1}$.

Recently, Selsing et al. (2019) published the X-shooter GRB optical afterglow legacy sample, which is the result of an extensive follow-up of 103 GRB afterglows with VLT/X-shooter between 2009 and 2017. Based on their work, we here aim to use a subsample of 22 GRBs at redshifts $z>2$ to perform a systematic search for absorption lines from molecular hydrogen, in order to answer the question whether, and if so why, there is a lack of $\mathrm{H}_{2}$ in GRB-DLAs. A full exploration of the column densities of the entire X-shooter sample will be presented in Thöne (in prep.). For the purpose of the present paper, we only

\footnotetext{
1 Recently, the $\mathrm{CH}^{+}$molecule was also detected in the optical spectrum of GRB140506A at $z=0.889$ (Fynbo et al. 2014).
}

use such measurements to analyze the metal and dust depletion characteristics of the GRB-DLAs where $\mathrm{H}_{2}$ can be searched for.

The paper is organized as follows. In Sect. 2, we describe the $\mathrm{H}_{2}$ GRB afterglow X-shooter subsample, and in Sect. 3 the absorption line analysis methods we have developed. Our main results are summarized in Sect. 4 and discussed in Sect. 5. We conclude in Sect. 6. Throughout the paper, when referring to GRBs, we refer to the class of long-duration GRBs, and column densities are given in $\mathrm{cm}^{-2}$.

\section{Sample selection}

The GRB sample presented in this paper was selected based on the complete sample of GRB afterglows observed with X-shooter as presented in Selsing et al. (2019). This sample is selected to be unbiased with respect to the parent Swift GRB sample, while at the same time optimising observability. This was done on the basis of a set of selection criteria, as described in Selsing et al. (2019). Out of the full statistical sample, afterglow observations were possible for 93 bursts. From this complete sample of $93 \mathrm{GRBs}, 41$ are at redshift $z>1.7$, which allows measuring the neutral hydrogen column density (see their Table 4). From these 41 bursts, we further select the 33 bursts at redshift $z>2.0$, where enough Lyman and Werner lines of molecular hydrogen are shifted into the X-shooter UVB arm, which is required to properly constrain the $\mathrm{H}_{2}$ column density. This sample of 33 GRBs is presented in Table 1, where we list the individual redshifts as well as the instrumental resolution in each $\mathrm{X}$-shooter arm, as taken from Selsing et al. (2019), and also the $\mathrm{S} / \mathrm{N}$ measured in the range of the expected $\mathrm{H}_{2}$ absorption lines.

From these 33 bursts, however, only 22 have X-shooter spectra with a $\mathrm{S} / \mathrm{N}$ ratio that is high enough to perform a rigorous analysis of the $\mathrm{H}_{2}$ absorption lines $(S / N \geq 2)$. Therefore, 11 bursts were excluded from the final sample and are consequently listed at the bottom of the table, including a small note indicating the potential reason for the $\mathrm{S} / \mathrm{N}$ being so low. This selection does not come without possible biases, which are important to address, and which we discuss below.

First of all, it is usually found that about 20 to $40 \%$ of the GRB population, the so-called dark bursts, are behind significant amounts of dust $\left(A_{V}>0.5 \mathrm{mag}\right)$ along the GRB host galaxies' line of sight (Perley et al. 2011; Greiner et al. 2011; Covino et al. 2013), although we note that the fraction might get lower at redshift $z>4$ (Bolmer et al. 2018; Zafar et al. 2018a). This is important, because the attenuation of the GRB afterglow by dust usually increases from red to blue wavelengths, which has a stronger effect on the observed spectrum with increasing redshift of the GRB, meaning that dusty and/or high redshift bursts are likely missed in spectral follow-up campaigns. For example, GRB 110128A, 130612A, and 161014A, that were excluded from the sample, were already very faint only several hours after the GRB trigger and might, therefore, be behind substantial dust columns. And, since all 22 GRBs in our sample are only behind small to moderate amounts of dust $\left(A_{V}<0.5\right.$ mag, see Table A.3), our sample might very well be biased toward sightlines with small foreground host galaxy dust columns. All the other burst that were excluded from the sample could only be observed later than $T_{0}+12 \mathrm{~h}$ or were observed under poor seeing conditions, and their exclusion should therefore not add any biases to the sample selection.

Another bias, however, might be introduced by including GRBs at redshift $z>4$, where the Lyman- $\alpha$ forest becomes an increasing hindrance in detecting the absorption lines from 
Table 1. Our sample of 22 GRB afterglows at $z>2$ observed with $\mathrm{X}$-shooter.

\begin{tabular}{|c|c|c|c|c|c|}
\hline \multirow{2}{*}{$\begin{array}{c}\text { GRB } \\
\text { yymmdd\# }\end{array}$} & \multirow{2}{*}{$\begin{array}{c}\text { Redshift } \\
(z)\end{array}$} & \multicolumn{3}{|c|}{ Instrumental resolution $(F W H M)$} & \multirow[t]{2}{*}{$S / N$} \\
\hline & & $\operatorname{UVB}\left(\mathrm{km} \mathrm{s}^{-1}\right)$ & $\operatorname{VIS}\left(\mathrm{km} \mathrm{s}^{-1}\right)$ & $\operatorname{NIR}\left(\mathrm{km} \mathrm{s}^{-1}\right)$ & \\
\hline 090809A & 2.7373 & 50.8 & 29.4 & 44.8 & 3 \\
\hline 090926A & 2.1069 & 49.1 & 28.4 & 42.2 & 14 \\
\hline 100219A & 4.6676 & 48.7 & 28.7 & 46.9 & 3 \\
\hline $111008 \mathrm{~A}$ & 4.9910 & - & 22.7 & 38.5 & 5 \\
\hline $111107 \mathrm{~A}$ & 2.8930 & 49.3 & 20.6 & 40.5 & 4 \\
\hline $120327 \mathrm{~A}$ & 2.8143 & 47.9 & 29.0 & 41.0 & 15 \\
\hline $120712 \mathrm{~A}$ & 4.1719 & 53.2 & 34.5 & 55.6 & 2 \\
\hline 120716A & 2.4874 & 56.1 & 33.0 & 48.9 & 4 \\
\hline $120815 \mathrm{~A}$ & 2.3582 & 48.6 & 26.8 & 48.4 & 6 \\
\hline 120909A & 3.9290 & 60.1 & 32.0 & 55.6 & 11 \\
\hline $121024 \mathrm{~A}$ & 2.3005 & 50.2 & 24.6 & 38.0 & 5 \\
\hline 130408A & 3.7579 & 50.6 & 22.9 & 38.5 & 12 \\
\hline $130606 \mathrm{~A}$ & 5.9127 & - & 24.2 & 46.2 & 15 \\
\hline $140311 \mathrm{~A}$ & 4.9550 & - & 28.5 & 40.4 & 3 \\
\hline $141028 \mathrm{~A}$ & 2.3333 & 51.2 & 30.6 & 44.7 & 4 \\
\hline 141109A & 2.9940 & 51.7 & 29.9 & 44.9 & 9 \\
\hline $150403 \mathrm{~A}$ & 2.0571 & 51.9 & 29.9 & 44.9 & 6 \\
\hline $151021 \mathrm{~A}$ & 2.3297 & 52.7 & 28.3 & 46.9 & 3 \\
\hline 151027B & 4.0650 & 54.3 & 31.2 & 47.3 & 8 \\
\hline $160203 \mathrm{~A}$ & 3.5187 & 51.5 & 23.1 & 38.5 & 14 \\
\hline $161023 \mathrm{~A}$ & 2.7100 & 50.7 & 29.3 & 44.0 & 16 \\
\hline $170202 \mathrm{~A}$ & 3.6456 & 46.9 & 27.3 & 40.0 & 9 \\
\hline \multicolumn{2}{|c|}{ Excluded } & \multicolumn{3}{|c|}{ Comment } & \\
\hline 100728B & 2.106 & \multirow{2}{*}{\multicolumn{3}{|c|}{$\begin{array}{l}\text { Obs. } \mathrm{T}_{0}+22.0 \mathrm{~h} \\
\text { Dark burst? high } A_{V} ?\end{array}$}} & $<2$ \\
\hline $110128 \mathrm{~A}$ & 2.339 & & $A_{V} ?$ & & $<2$ \\
\hline $120404 \mathrm{~A}$ & 2.876 & \multicolumn{3}{|c|}{ Obs. $\mathrm{T}_{0}+15.7 \mathrm{~h}, A_{V} \sim 0.07 \mathrm{mag}$} & $<2$ \\
\hline $121201 \mathrm{~A}$ & 3.385 & \multicolumn{3}{|c|}{ Obs. $\mathrm{T}_{0}+12.0 \mathrm{~h}, A_{V} \sim 0.17 \mathrm{mag}$} & $<2$ \\
\hline $121229 \mathrm{~A}$ & 2.707 & \multicolumn{3}{|c|}{ Poor seeing } & $<2$ \\
\hline 130427B & 2.780 & \multicolumn{3}{|c|}{ Obs. $\mathrm{T}_{0}+20.6 \mathrm{~h}$, twilight } & $<2$ \\
\hline $130612 \mathrm{~A}$ & 2.007 & \multicolumn{3}{|c|}{ Dark burst? high $A_{V}$ ? } & $<2$ \\
\hline 131117A & 4.042 & \multicolumn{3}{|c|}{$A_{V}<0.20 \mathrm{mag}, z>4$} & $<2$ \\
\hline $140515 \mathrm{~A}$ & 6.327 & \multicolumn{3}{|c|}{ Obs. $\mathrm{T}_{0}+15.5 \mathrm{~h}, z>6$} & $<2$ \\
\hline $140614 \mathrm{~A}$ & 4.233 & \multicolumn{3}{|c|}{$A_{V}<0.50 \mathrm{mag}, z>4$} & $<2$ \\
\hline 161014A & 2.823 & \multicolumn{3}{|c|}{ Dark burst? anomalous extinction? } & $<2$ \\
\hline
\end{tabular}

Notes. Out of the 33 initially selected bursts, 11 were excluded because of poor $\mathrm{S} / \mathrm{N}$, which are additionally listed at the bottom of the table. Columns from left to right: name of the GRB; redshift; FWHM of the instrumental resolution in $\mathrm{km} \mathrm{s}^{-1}$ inferred in the UVB, VIS, and NIR arms of X-shooter (taken from Selsing et al. 2019); S/N in the range of the expected $\mathrm{H}_{2}$ absorption lines. For the 11 bursts at the end of the table that were excluded from the sample, we write a small comment indicating the potential reason for the $\mathrm{S} / \mathrm{N}$ being so low.

molecular hydrogen, which all fall blue-wards of $1215 \AA$ A. Also, at redshift $z>4$ the Lyman blanketing significantly reduces the observed flux bluewards of Ly $\alpha$. This is the case for 6 of the 22 GRBs (27\%), which will thus be discussed and labeled separately throughout the paper. Also, three of the excluded bursts are at redshift $z>4$.

\section{Methods}

Kinematic or velocity profiles of absorption lines are a powerful tool to analyze the different gas phases in DLA absorbers. The predominant absorption features imprinted on the afterglow spectrum of the GRB are from singly ionized metal species, residing in the cold, neutral gas of the GRB host galaxy. In most cases, also absorption lines from higher ionization species are seen in the spectra, which trace the warm, ionized gas and therefore usually have a different kinematic profile, offset to the singly-ionized lines (Fox et al. 2008; Wiseman et al. 2017a; Heintz et al. 2018). Since we are only interested in the neutral gas phase, these high ionization species will not be considered in this paper.

To analyze and FIT the absorption lines in the afterglow spectra in our sample of 22 GRBs, we developed our own, stateof-the-art routines, which are entirely written in python and are based on the Markov chain Monte Carlo (MCMC) Bayesian inference library PyMC 2.3.7 .

Absorption lines are usually modeled with Voigt line profiles, which are fitted to the spectra simultaneously with the continuum flux of the GRB. The model for such an absorption profile in velocity space with a number of $n$ absorption components is described by

$F_{\text {model }}=F_{\mathrm{c}} \cdot \prod_{j=1}^{n} e^{-\tau_{j}} ; \quad F_{\mathrm{c}}=A+B v+C v^{2}$

where the optical depth $\tau$ is given by

$$
\tau=\frac{\pi e^{2}}{m_{\mathrm{e}} c} f_{i k} \lambda_{i k} N \cdot \phi_{V}\left(v-v_{0}, \frac{b}{\sqrt{2}}, \Gamma\right),
$$

where $\phi_{V}$ is the Voigt profile in velocity space, and with $f_{i k}$, $\lambda_{i k}$ and $\Gamma$, being the oscillator strength, wavelength and damping constant for transition $i \rightarrow k$. The relative velocity of the absorption component $v_{0}$, the broadening parameter $b$, the column density $N$, and possibly also the number of components $n$, are the parameters of interest for our analysis. For the continuum flux of the GRB $F_{\mathrm{c}}$, which is usually best described by a simple power law, we assume a second-order polynomial, which introduces another three free parameters $(A, B$, and $C)$. The Voigt profile $\phi_{V}$ is a convolution of a Gaussian (thermal and turbulent motion) and a Lorentzian (uncertainty of transition frequencies) profile, and is related to the real part of the Faddeeva function $w(z)$ that we imported from the Python package $\mathrm{SciPy}^{3}$. Finally, the absorption line profile is convolved with a Gaussian with a FWHM of the instrumental resolution in the given arm, as determined by Selsing et al. (2019; see Table 1).

$\phi_{V}=\frac{\operatorname{Re}(w(z))}{b \sqrt{\pi}} ; \quad z=\frac{v-v_{0}+i \Gamma}{b}$.

Given this model, our parameters of interest are constrained in the following way. First, we select transitions that display a distinct absorption profile, that is unblended lines that fall in a segment of the spectrum with a good S/N, usually Si II 1808.0129, Fe II 1608.4509, or Mg II 2803.5311, and compare them in order to identify the number of required absorption components. This is done by fitting these lines with multiple MCMC runs for a realistic range of the number $n$ of possible absorption components with each of a total of 100000 iterations and with a warm-up phase of 95000 iterations. For DLAs with three or more distinct absorption components we doubled or tripled the number of iterations, and for the relative velocity of each component we start by putting a uniform prior on the whole input velocity range, which was usually $v_{0} \pm 1000 \mathrm{~km} \mathrm{~s}^{-1}$. After each run, we analyzed the resulting FIT and posterior distributions and compared the models for a different number of components by calculating $\chi^{2}$ until a convincing solution was

\footnotetext{
2 https://pymc-devs.github.io/pymc

3 https://docs.scipy.org/doc/scipy/reference/ generated/scipy.special.wofz.html
} 

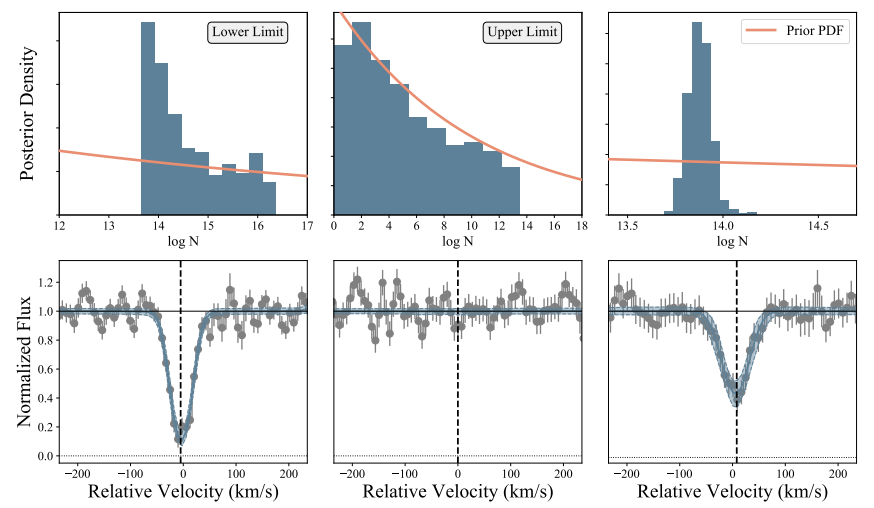

Fig. 1. Typical posterior distributions for a saturated line, a nondetection, and a line which can be used to derive a column density accurately. In the case of a non-detection, the posterior distribution is completely determined by the prior PDF, which is expected in case there is in the data no information about the assumed model, in this case a Voigt-profile-like absorption.

found. Once the required number $n$ and the relative velocity $v_{0}$ of each absorption component were identified by also comparing the results from different lines, we fixed $n$ and continued to FIT the other lines by using smaller uniform priors on $v_{0}$, usually allowing for a small variation of $v_{0} \pm 20 \mathrm{~km} \mathrm{~s}^{-1}$. For the broadening parameter, we generally also used uniform priors between the minimum resolvable value, usually $5-15 \mathrm{~km} \mathrm{~s}^{-1}$, and a maximum value of $80 \mathrm{~km} \mathrm{~s}^{-1}$. Finally, for the column density, we chose an exponential prior, which ensures that lower column densities are preferred when there is no information about absorption in the data, that it the flux around the position of the expected absorption line is consistent with the noise. As an example, in Fig. 1, we show the posterior distributions for the column density of a single absorption line in three different cases: a saturated line, a non-detection, and a line which can be used to measure a column density.

As expected for the resolution of $\mathrm{X}$-shooter $(R \approx 4000$ 17000 or equivalently $75-18 \mathrm{~km} \mathrm{~s}^{-1}$ ), even mildly saturated lines show a strong degeneracy between the column density and the broadening parameter (hidden saturation; Prochaska 2006; Wiseman et al. 2017b), which to identify requires sufficient sampling of the parameter space, which is growing exponentially with increasing number of absorption components. The effect of hidden saturation is demonstrated in Fig. 2, where we show pairwise contour plots and posteriors distributions of the three parameters from the saturated line shown in Fig. 1. Since the column density and the broadening parameter are highly correlated we use the adaptive Metropolis algorithm implemented in PyMC for these parameters ${ }^{4}$. For all other parameters, also the three parameters from the second-order polynomial for the background flux (normal priors around $A=1, B=0$ and $C=0$ with variance $\sigma=0.5$ ), we use the MetropolisHastings step method. For the likelihood, we chose a Gaussian whose standard deviation is equal to the flux error. Finally, we want to note, that for the resolution of X-shooter, individual absorption components are often blended with each other, and therefore the decomposition of the profile is not necessarily unambiguous, such that the resultant $b$-values might not be physical.

4 For a general introduction on MCMC methods and algorithms for Bayesian data analysis, we refer the reader to Betancourt (2017) or Sharma (2017) and the references therein.

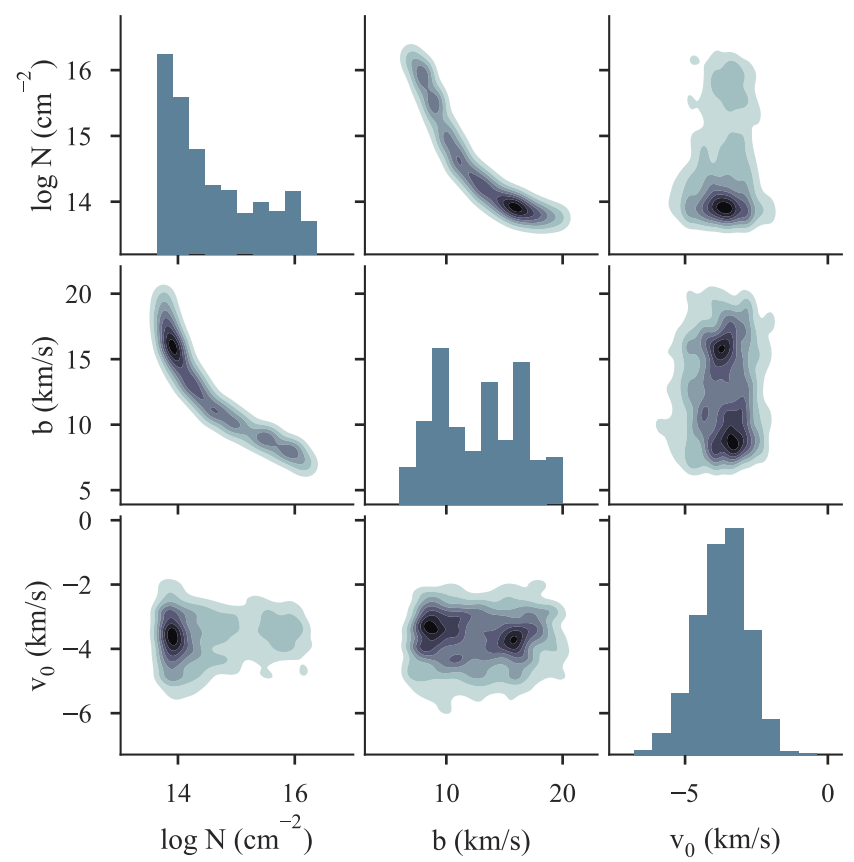

Fig. 2. Pairwise contour plots and posterior distributions for the column density, broadening parameter, and velocity of the saturated line shown as example in Fig. 1.

\section{Results}

\subsection{Neutral hydrogen and metal abundances}

The most prominent absorption feature in GRB afterglow spectra is the Lyman- $\alpha$ line, which is used to measure the neutral hydrogen column density $N(\mathrm{H} \mathrm{I})$. For each of the 22 bursts in the selected sample, $N(\mathrm{H}$ I) was previously inferred by Selsing et al. (2019), by simply plotting synthetic absorption lines over the normalized spectra until a satisfying match was reached (which is a standard procedure, e.g., Fynbo et al. 2009). We are able to derive slightly more accurate values by fitting the Lyman- $\alpha$ line by also including all absorption lines in the red and blue damping wing. The results are listed in Table A.1 and shown in Fig. 4. Those parts of the spectrum that were ignored for the FIT, mostly due to telluric and Ly $\alpha$ forest lines or too complex absorption structures, are marked by the gray shaded areas. To compare the results from both methods, in Fig. 3, we additionally plot the values derived in this paper versus those from Selsing et al. (2019). Besides one outlier, GRB 120712A, which has the lowest $\mathrm{S} / \mathrm{N}$, all values are consistent within in the errors, confirming the robustness of both methods.

Furthermore, to measure the metallicity and study the dust depletion in each system, for all 22 GRB spectra we also FIT lines and measure column densities of ten different metal species, O I, Zn II, S II, Si II, Mg II, Mn II, Cr II, Ni II, Fe II, and Ti II, at least in those cases where it is possible and the spectrum is not affected by, for example, strong telluric lines. Besides Ti II, all of these absorption lines are commonly detected in GRB afterglow spectra (Christensen et al. 2011). In case of nondetections or saturated lines we determine 3- $\sigma$ upper or lower limits, respectively. All measured column densities, as well as upper or lower limits, are listed in Table A.1 and the corresponding plots showing the most constraining fits for each burst are shown in Figs. B.1-B.22.

The values for the oscillator strength $f$ and damping constant $\Gamma$ are taken from the atomic data file (atom.dat) which is 


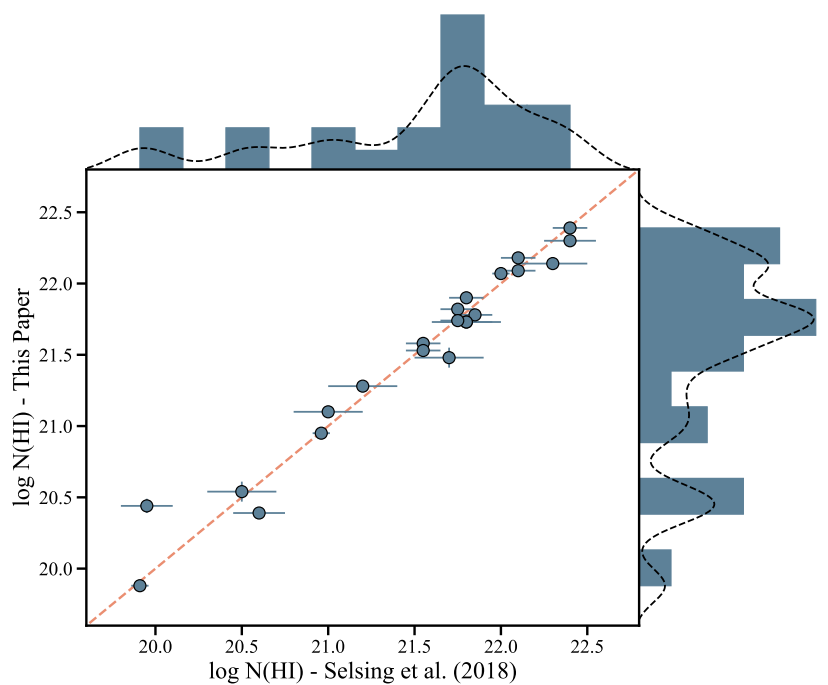

Fig. 3. Neutral atomic-hydrogen column densities determined in this paper versus those from Selsing et al. (2019). The dashed line is the line of equality. Additionally, we show histograms and corresponding kernel density estimates for both measurement sets.

distributed in VPFIT version 10.2 and originally based on Morton (2003). For Zn II and S II, however, we use the updated oscillator strength values from Kisielius et al. (2014, 2015) instead. While the difference in case of S II is almost negligible, the column densities of $\mathrm{Zn}$ II are about 0.1 dex lower when using the new values. We also note that in most cases, the three sulfur lines $(\lambda=1250.58,1253.81$, and $1259.52 \AA)$ were fitted simultaneously with $\operatorname{Ly} \alpha$, because they fall into the red part of the damping wing for column densities larger than $\log N(\mathrm{HI}) \gtrsim 21.0$, which is true for the majority of the bursts in the sample (see Fig. 4). Finally, since the two Zn II lines at $\lambda=$ 2026.14 and $2062.66 \AA$ are blended with lines from $\mathrm{Cr}$ II and $\mathrm{Mg}$ I, we FIT all of these lines simultaneously.

To compare the column densities measured in this paper with those previously published in the literature, in Fig. 5, we plot our values compared to those collected from Wiseman et al. (2017b). The latter are partially determined by Wiseman et al. (2017b) and otherwise taken from different papers, i.e.: GRB 090809 from Skúladóttir (2010), 090926A from D'Elia et al. (2010), 100219A from Thöne et al. (2013), 111008A from Sparre et al. (2014), 120327A from D'Elia et al. (2014), 120815A from Krühler et al. (2013), and 121024A from Friis et al. (2015). Additionally, we also plot the values for GRB 161023A from de Ugarte Postigo et al. (2018). Since we are able to better identify any strong degeneracies in the $N$ versus $b$ parameters space, we are generally more conservative and put more upper or lower limits on the column densities compared to previous published values, which are not plotted in Fig. 5. Otherwise, our measurements are in reasonable agreement with the previous published values. The major differences can be explained by the updated oscillator strength values in case of $\mathrm{Zn} \mathrm{II}$, a better exploration of the parameter space in case of blended lines (Zn II and Cr II), or the in general advanced analysis method, which allows a better and more efficient exploration of the parameter space compared to the least squared approach of VPFIT.

\subsection{Dust depletion}

Due to their apparent faintness, most of our knowledge on the elemental abundances in high redshift galaxies $(z>2)$, originates from absorption line studies of DLA systems in the spectra of QSOs and GRBs (Schady 2017) $)^{5}$. However, refractory elements, like $\mathrm{Fe}$, $\mathrm{Ni}$ or $\mathrm{Cr}$, can be heavily depleted by condensation onto interstellar dust grains, with different strength of the depletion depending on the element and the sight-line (Savage \& Sembach 1996; Jenkins 2009; De Cia et al. 2016). Thus, if some elements are partially locked onto dust grains, they are not completely accounted for when measuring the gas-phase abundances using absorption line analysis, and we, therefore, have to analyze the depletion in each sight-line independently. The analysis of the dust depletion can thus be used to measure the dust content in the GRB host galaxies line of sight and to determine the dust-corrected metallicity $[\mathrm{M} / \mathrm{H}]$ of the DLA.

As a first indicator of the strength of depletion, one can calculate the depletion factor $[\mathrm{Y} / \mathrm{Fe}] \equiv \log (N(\mathrm{Y}) / N(\mathrm{Fe}))-$ $\log (N(\mathrm{Y}) / N(\mathrm{Fe}))_{\odot}$, where $\mathrm{Y}$ is a non-refractory element that is only marginally depleted onto dust grains, like $\mathrm{Zn}$ or $\mathrm{S}$, and is compared to iron, that is usually heavily depleted.

A more sophisticated way to study the dust depletion in each of the GRB-DLAs, is to compare the relative abundances of multiple refractory and non-refractory elements and use relations found in the Milky Way and QSO-DLAs to interpret the depletion pattern (Jenkins 2009; De Cia et al. 2013, 2016; Wiseman et al. 2017b). This method can be justified by the homogeneous properties regarding the metal and dust content found for different environments and redshift, that indicate that most of the dust content is built up from grain growth in the ISM, independent of the specific star formation history (De Cia 2018). To calculate the relative abundances $[\mathrm{X} / \mathrm{H}] \equiv \log (N(\mathrm{X}) / N(\mathrm{H}))-\log (\mathrm{X} / \mathrm{H})_{\odot}$ we adopt the solar abundances from Asplund et al. (2009), following the recommendations of Lodders et al. (2009), as listed in Table 1 of De Cia et al. (2016). We also assume that our measurement of the column density of an element that is singly ionized is representative of the total gas-phase abundance of that element. This is justified by the large pool of neutral gas $\log N(\mathrm{HI}) \gtrsim 20.3$, found for all GRBs in our sample besides 130606A, indicating that only a small, negligible fraction of higher ionized elements should exist (Wolfe et al. 2005; Kanekar et al. 2011).

After calculating the relative abundances, our analysis of the dust depletion sequences was done following the work of Wiseman et al. (2017b), which is based on the results from De Cia et al. (2016). However, instead of using their minimum least squares approach, we again use a Bayesian framework and sample the posterior distributions using PyMC. For the depletion strength factor we use a uniform prior between $F_{*}=-2.5$ and 1.0 and for the dust-corrected metallicity of the system a uniform prior between $[\mathrm{M} / \mathrm{H}]=-3$ and $1.0^{6}$. We then run an MCMC with 100000 iterations and sample the posterior distributions with the last 10000 iterations after a warm-up phase of 90000 iterations. The model for the expected relative abundance in element $\mathrm{X}$ is given by

$[\mathrm{X} / \mathrm{H}]_{\exp }=A+B \frac{\left(F_{*}-1.50\right)}{1.48}+[\mathrm{M} / \mathrm{H}]$

where $A$ and $B$ are the updated linear depletion parameters taken from De Cia et al. (2016). The best-FIT $F_{*}$ and $[\mathrm{M} / \mathrm{H}]$ can then be used to determine the dust-to-metals ratio $\mathcal{D T M}$ (normalized to the Milky Way), and further the expected visual extinction

5 First attempts have been made to also use superluminous supernova as background sources to study high-redshift galaxies in absorption (Vreeswijk et al. 2014; Yan et al. 2018).

$6 \quad F_{*}$ represents the strength of dust depletion and is defined by Jenkins (2009) to range between 0 and 1 for the Galactic ISM. 


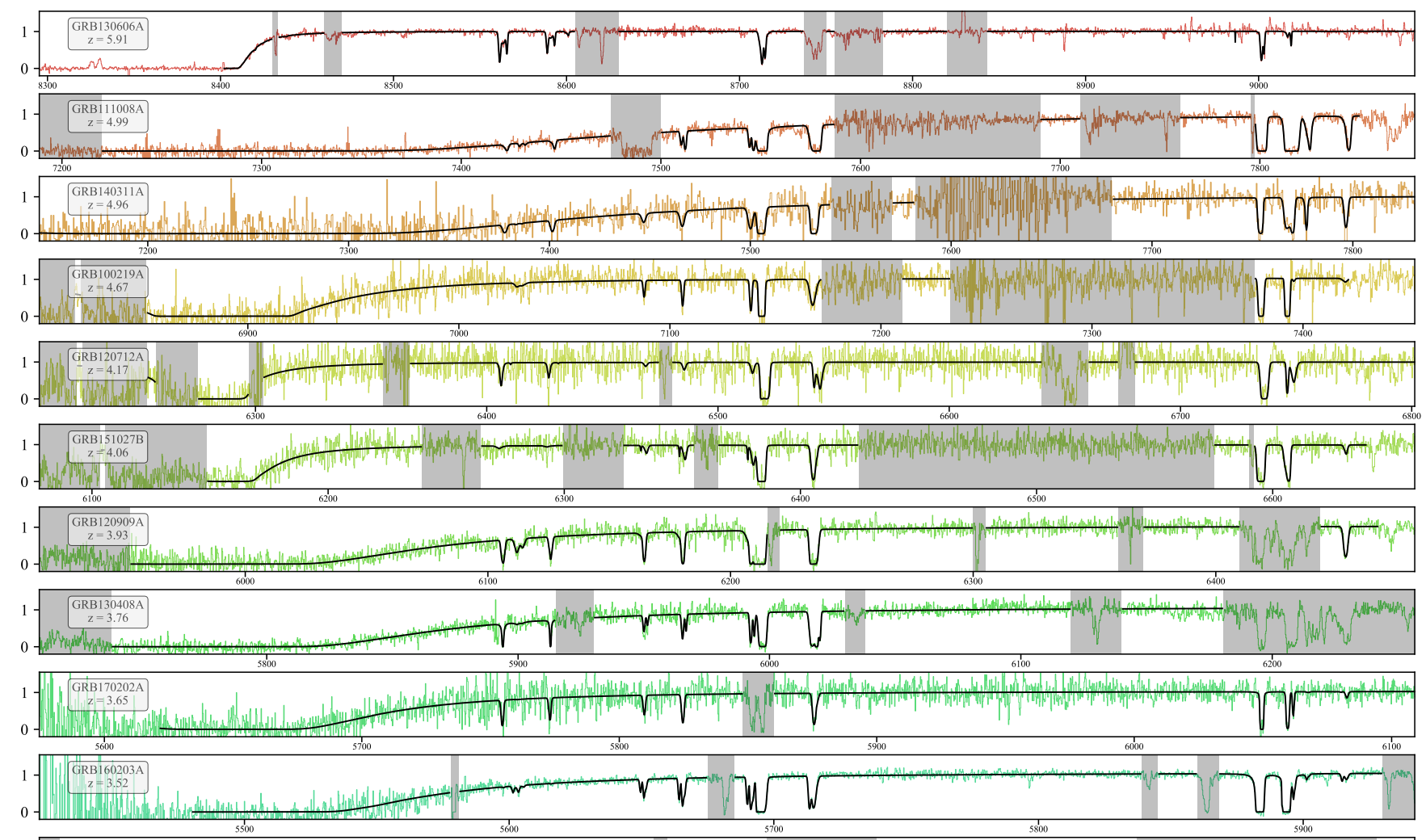

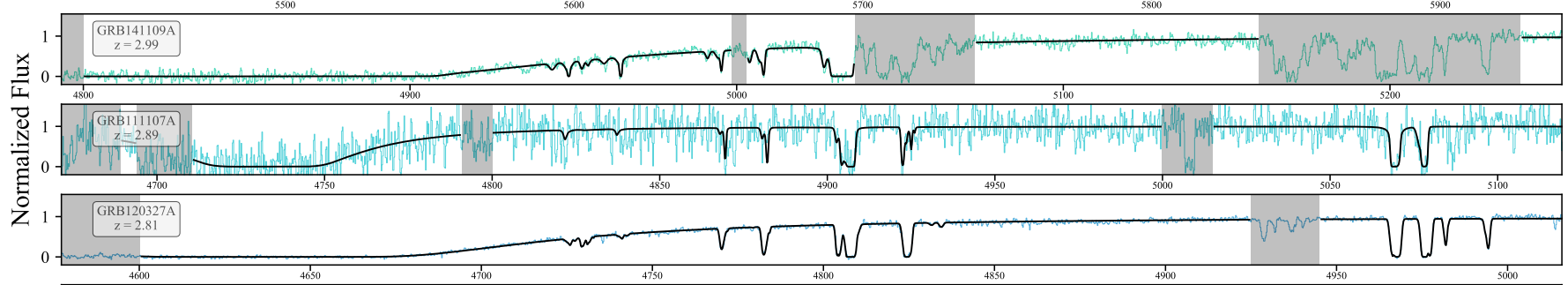

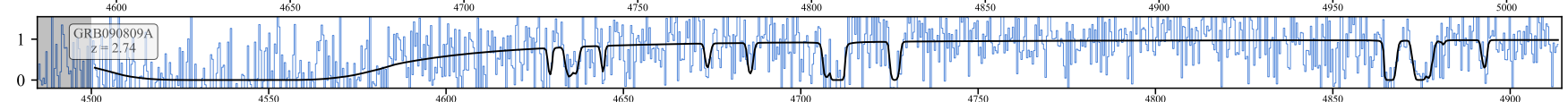

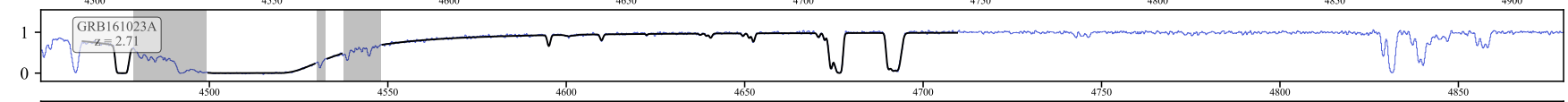

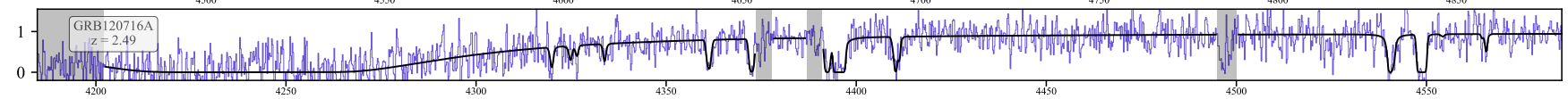

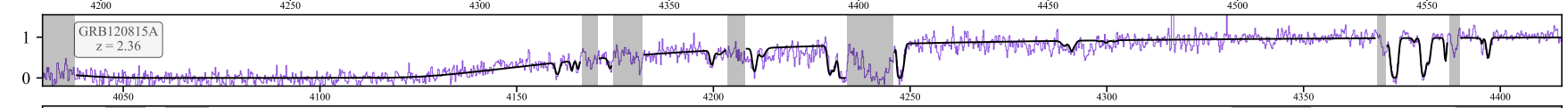

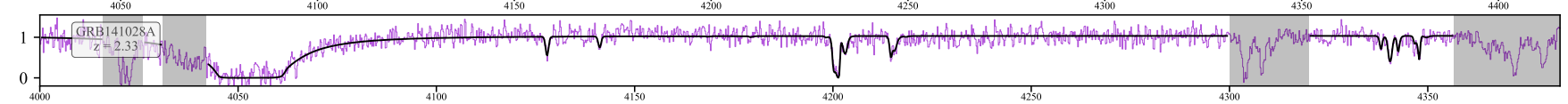

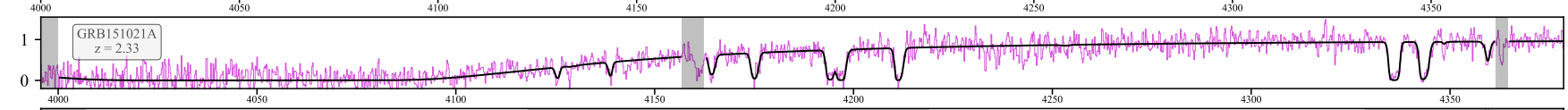

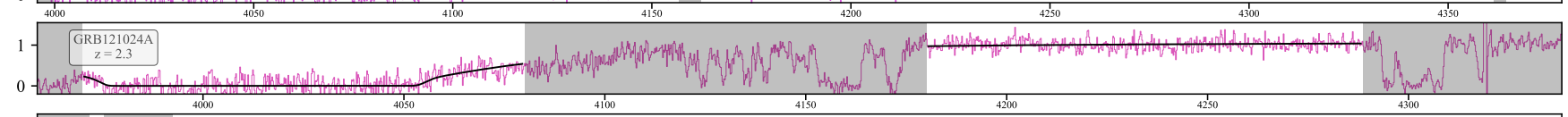

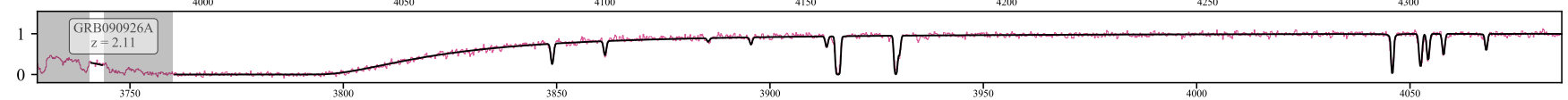

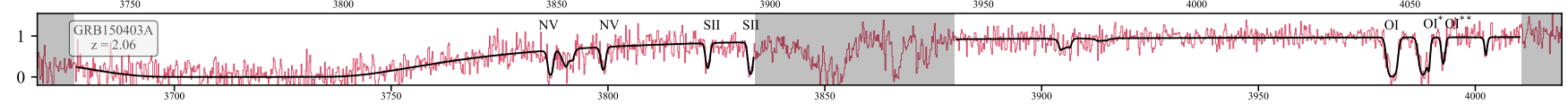

Observed Wavelength $(\AA)$

Fig. 4. Normalized X-shooter spectra around the observed Lyman- $\alpha$ absorption lines for the 22 GRB afterglows analysed in this paper ordered by decreasing redshifts from top to bottom panels. The best-FIT model is shown in each case by a solid black line. The gray shaded areas indicate the regions that were masked out during the fits, usually due to the interposition of telluric lines or complex absorbing structures. 


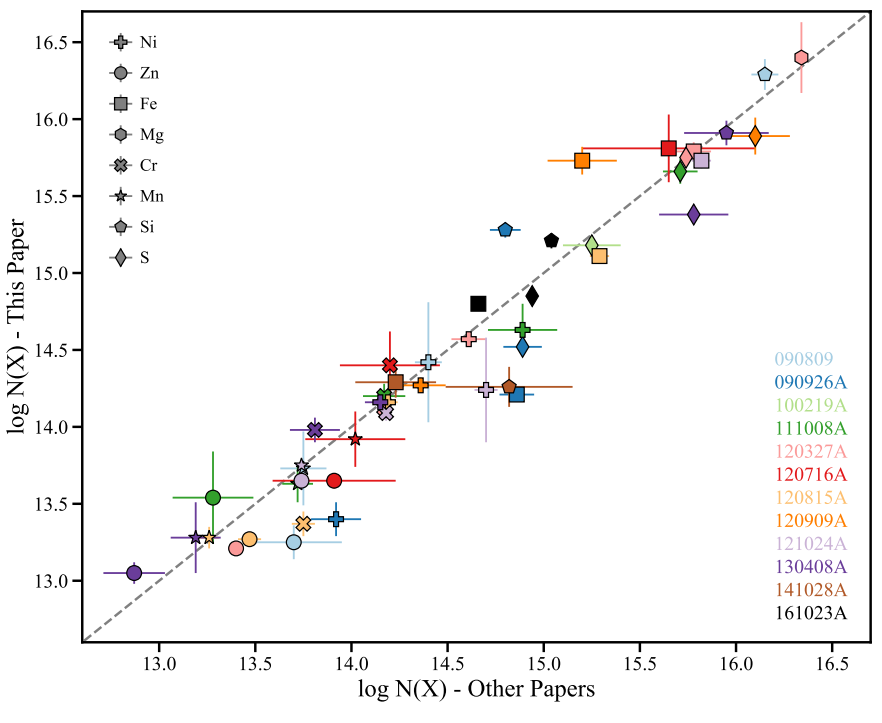

Fig. 5. Metal column densities derived in this paper versus those published in Wiseman et al. (2017b), which are partially compiled from various sources. Also plotted are the values for GRB 161023A from de Ugarte Postigo et al. (2018). The dashed line is the line of equality. Different metal species $\mathrm{X}$ are indicated using different symbols (as labeled in the figure).

$A_{V}$ (depl.) in the host galaxy line of sight. The dust-to-metals ratio is the ratio between the total dust column density in terms of atoms in the dust phase and the total metal column density, which is further normalized to the Milky Way value. It is derived using Eq. (C.3) of De Cia et al. (2013) and assuming an average $[\mathrm{Fe} / \mathrm{Zn}]_{\mathrm{Gal}}=1.22$ found in Galactic sight-lines (Jenkins 2009). $A_{V}$ (depl.) is calculated using the average extinction for a given hydrogen column density in the Milky Way, and using the $N(\mathrm{H}) / A_{V}$ derived by Watson (2011; for more details, see Sects. 4.2 and 6.2 in Wiseman et al. 2017b, and also Savaglio \& Fall 2004 and De Cia et al. 2016). For all 22 GRBs, the results of the best-FIT depletion pattern, as well as the corresponding contour plots for the dust-corrected metallicity $[\mathrm{M} / \mathrm{H}]$ versus the depletion strength factor $F_{*}$, are shown in Fig. 6 and the bestFIT results are listed in Table A.3. Finally, we note that elements that are intrinsically under- or over-abundant, can badly influence the results of the depletion analysis in those cases where only a few elements are available. Also, the negative values of $F^{*}$ we find in DLAs are based on the assumption of a linear relation between $[\mathrm{Zn} / \mathrm{Fe}]$ and $F_{*}$ which is extrapolated at negative $F_{*}$.

A detailed analysis of nucleosynthesis effects is beyond the scope of this paper and may be studied in a forthcoming paper.

\subsection{Visual extinction}

Measurements of the host intrinsic visual extinction from fitting extinction curves to the afterglow SED, $A_{V}$ (SED), are available in the literature for all bursts from our $\mathrm{H}_{2}$ sample. The individual values and corresponding references are listed in Table A.3. Most of them were taken from Bolmer et al. (2018), in case of the GRBs at $z>4$, or from the measurements for the GROND $4 \mathrm{~h}$ sample (Greiner et al. 2011; Greiner, in prep.).

Following Wiseman et al. (2017b), in Fig. 7, we plot the host intrinsic visual extinction as derived from the depletion analysis $A_{V}$ (depl.) versus the measurement from the SED FIT $A_{V}$ (SED). Similar to Wiseman et al. (2017b) and Savaglio \& Fall (2004), we find that for some of the sight-lines the values are not consistent with being identical, and that data points can fall both below and above the line of equality. In contrast to Wiseman et al. (2017b), however, we find a better agreement between both methods, at least within the errors. Interestingly, most of the GRBs for which the extinction measured from the SED FIT is higher are from $z>4$. This can thus be explained by the higher probability of intervening systems in the lines of sight, which can contain dust that only contributes to the extinction derived from the SED FIT (Ellison et al. 2006; Ménard \& Fukugita 2012). In addition, we note that the depletion-derived $A_{V}$ only probes non-carbonaceous dust, for example, silicates or iron-rich grains, because it is based on the measurements of several metals, but not C. One possibility is therefore also that the $z>4$ systems contain more C-rich dust, such as amorphous carbons or Polycyclic Aromatic Hydrocarbons (PAH). At $z>4$, cosmic effects may importantly affect the dust composition, because of the less amount of heavy metals in the diffuse ISM which would increase the significance of C-rich molecular clouds as dust sources (Heintz, in prep.). Also, there is less cosmic time for the build up of dust grains, potentially making $\mathrm{SNe}$ more important dust producers at high redshift (Todini \& Ferrara 2001; Vladilo et al. 2018).

Data points that fall above the line of equality, that is sightlines for which the depletion derived value is higher, could be the result of additional uncertainties coming from SED fitting; that is from the choice of extinction laws and whether the SED is fitted with a simple or broken power law (see also the discussion in Wiseman et al. 2017b). Due to these reasons, and also because the $A_{V}$ (SED) values are collected from different works and are thus not derived as consistently as the $A_{V}$ (depl.) values in this work, we believe that the latter is a better estimate for the GRB host galaxies' line of sight, and we will therefore in the following only discuss our results in respect to $A_{V}$ (depl.) where necessary.

\subsection{Dust-corrected metallicities}

In Fig. 8, we plot the dust-corrected metallicity $[\mathrm{M} / \mathrm{H}]$ as derived from the depletion analysis versus the metallicity derived using a single line of an element $\mathrm{X}$ with low depletion $[\mathrm{X} / \mathrm{H}]$. As previously found by, for example, De Cia et al. (2018), we find that even $\mathrm{Zn}$ is slightly depleted onto dust grains, which explains that almost all of the data points fall above the line of equality. The two exception are GRB 111008A and 140311, which are both at redshift $z>4$. Looking at the depletion pattern of these two bursts, it appears that $\mathrm{Zn}$ is intrinsically over-abundant compared to $\mathrm{Fe}$, or more likely that $\mathrm{Mn}$, which is an odd-Z element known to be intrinsically under-abundant in QSO-DLAs, is pulling the FIT down.

On average, the dust-corrected metallicities are with $\overline{[\mathrm{M} / \mathrm{H}]}=-1.09 \pm 0.50$ on about $0.2 \mathrm{dex}$ higher than those derived from $\mathrm{Zn}$ or $\mathrm{S}(\overline{[\mathrm{X} / \mathrm{H}]}=-1.27 \pm 0.37)$, but also have larger errors. Nevertheless, since dust corrections were not applied to the QSO-DLA samples we want to compare our GRB-DLAs against, we will for the further analysis only use $[\mathrm{X} / \mathrm{H}]$, but keep in mind that the actual metallicities might be slightly higher.

\subsection{Molecular hydrogen}

The Lyman and Werner absorption lines of molecular hydrogen, although numerous, are relatively hard to detect in the spectra of GRBs and QSOs, because they fall at rest frame wavelengths $\lambda<1150 \AA$, in the Lyman- $\alpha$ forest, where the spectrum is 

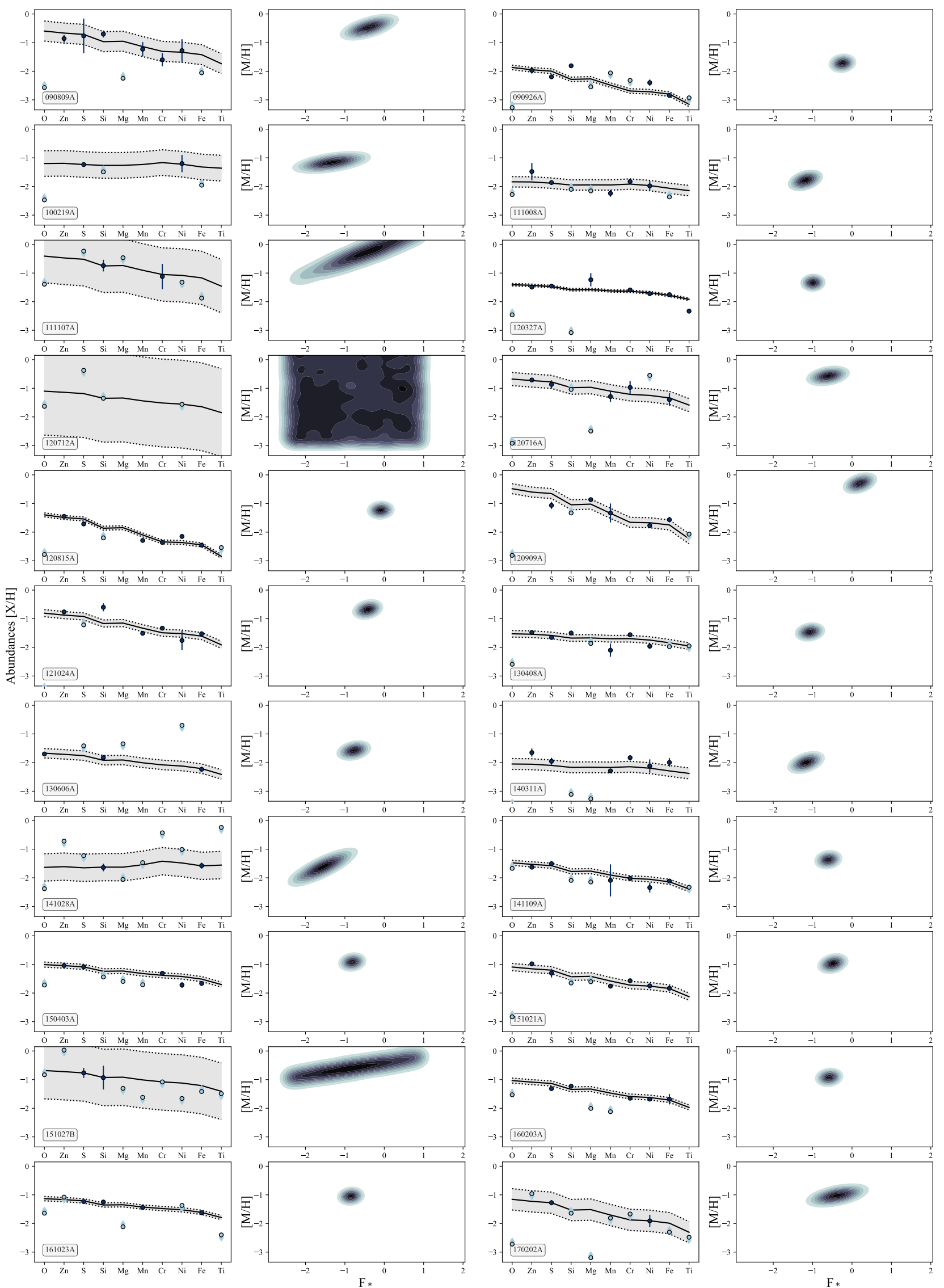

Fig. 6. Results from fitting dust depletion sequences for the 22 GRBs in our $\mathrm{H}_{2}$ sample. The relatives abundances of the elements used for the fits are plotted with dark blue dots. Upper and lower limits for the other elements are plotted in light blue. The black line indicates the best FIT, and the dashed lines and gray shaded areas the corresponding 3- $\sigma$ confidence intervals. A contour plot of dust-corrected metallicity $[\mathrm{M} / \mathrm{H}]$ versus $F_{*}$ is shown right next to every depletion sequence. 


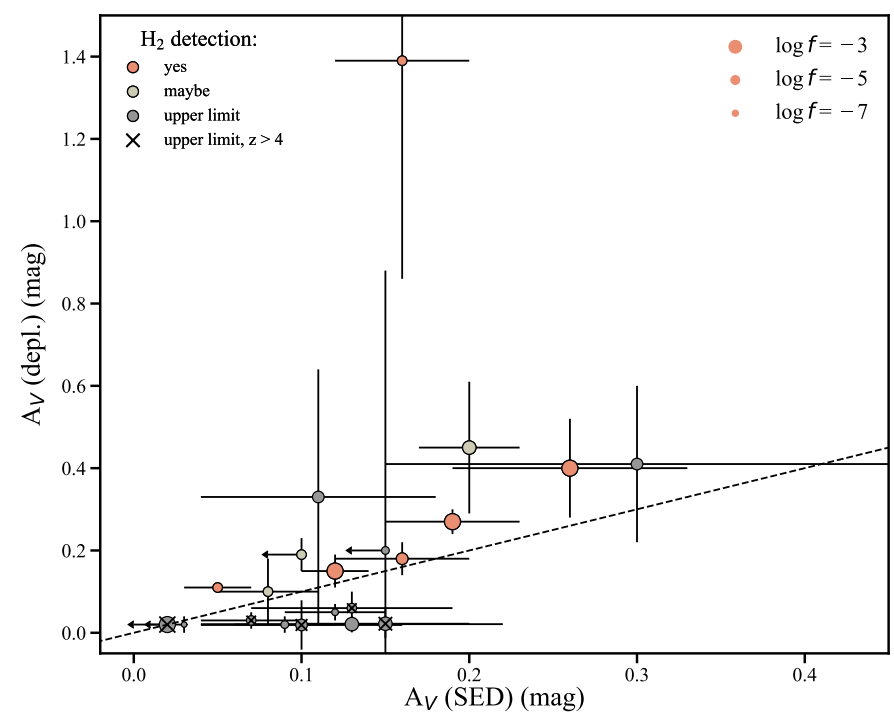

Fig. 7. Host-intrinsic visual extinction as measured from the depletion analysis $A_{V}$ (depl.) versus the measurement from a FIT to the spectral energy distribution in photometric data $A_{V}(\mathrm{SED})$. The dashed line is the line of equality.

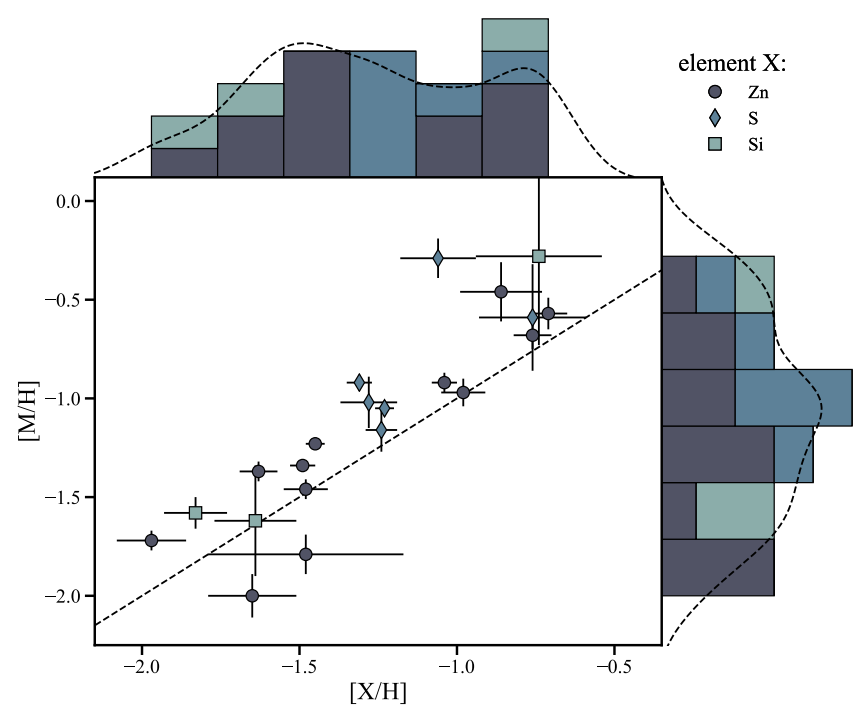

Fig. 8. Dust-corrected metallicity, $[\mathrm{M} / \mathrm{H}]$, as derived from the depletion analysis versus the metallicity derived using a single line of an element $\mathrm{X}$ with low depletion, $[\mathrm{X} / \mathrm{H}]$. For each burst, the used element is colored as labeled. The dashed line is the line of equality.

speckled with random absorption lines with column densities of $\log N(\mathrm{HI}) \lesssim 17$ (e.g., Kim et al. 2002). The number of these forest lines usually increases with redshift, and in case of additional absorption from dust in the GRB host galaxy or the Milky Way, the flux is additionally suppressed, especially at these blue wavelengths.

We therefore searched for absorption from molecular hydrogen by creating synthetic spectra with $\mathrm{H}_{2}$ absorption lines for different column densities $N\left(\mathrm{H}_{2}\right)$ and around different redshifts known from the low-ionisation lines and compared them with the normalized GRB spectra in both wavelength and velocity space. In the case of the comparison in velocity space, we plotted the strongest absorption lines on top of each other in order to look for coincidences. Following this method, we confirm the presence of molecular hydrogen lines in the previously known spectra, GRB 120327A, 120815A, and 121024A (Krühler et al. 2013; D'Elia et al. 2014; Friis et al. 2015). Additionally, we find conclusive evidence for absorption from $\mathrm{H}_{2}$ in three more systems, which are GRB 120909A, 141109A, and 150403A. For these six systems, we identified the most constraining lines in the rotational levels $J=0,1$, and 2, where most of the $\mathrm{H}_{2}$ is expected, and FIT them simultaneously. In cases where the observed velocity width of the $\mathrm{H}_{2}$ absorption lines is close to the instrumental resolution, we decided to measure the column density for both, a fixed broadening parameter of $b=2 \mathrm{~km} \mathrm{~s}^{-1}$ and $b=10 \mathrm{~km} \mathrm{~s}^{-1}$, in order to determine a realistic range for the total $\mathrm{H}_{2}$ column density. This is the case for four GRBs, namely GRB 120327A, 120909A, 121024A, and 141109A. For GRB 121024A the total molecular hydrogen column density remains unchanged regardless of the broadening parameter assumed, but for the other three burst the total molecular hydrogen column density is about $\Delta \log N\left(\mathrm{H}_{2}\right) \sim 2$ higher for $b=2 \mathrm{~km} \mathrm{~s}^{-1}$. However, for the following analysis, we will adopt the column densities measured for $b=2 \mathrm{~km} \mathrm{~s}^{-1}$, which is a more realistic value, given the temperatures of molecular gas. For the other two GRBs, 120815A and 150403A, because the lines are saturated, we are able to FIT a large part of the spectrum with all rotational levels simultaneously and by including other blending lines from, for example, Fe II as well (see Figs. 9 and 10).

Most of the other spectra do not show convincing evidence for absorption from molecular hydrogen. Some, however, are still consistent with relatively high column densities, but can only be labeled as possible detections, mostly because of poor $\mathrm{S} / \mathrm{N}$ or a strong Lyman- $\alpha$ forest. This is the case for three GRBs, namely GRB 151021A, 160203A, and 170202A. In Fig. 11, we show part of the spectrum of GRB 151021A, demonstrating the difficulty in claiming a detection of $\mathrm{H}_{2}$.

In these three cases and for the rest of the bursts for which we do not find any convincing evidence for absorption from molecular hydrogen in the spectra, we determine upper limits for the total molecular hydrogen column density with the following steps. First, we identify the three most constraining lines for the rotational levels $J=0,1$, and 2, FIT them simultaneously, and determine the upper limits as we did for metals lines and as described in Sect. 3. We then use these upper limits to create a synthetic spectrum, which we plot over the whole range of covered $\mathrm{H}_{2}$ lines in order to check if the determined upper limits are consistent with the rest of the spectrum. For some of the bursts we were not able to find regions of the spectrum where a proper FIT is possible, mostly because the $\mathrm{S} / \mathrm{N}$ is relatively poor, or the spectrum is covered with too many forest lines. In this case we create synthetic spectra with $\mathrm{H}_{2}$ absorption lines at the position of the strongest component of the metal lines, and increase the column density until the synthetic spectrum is not consistent with the data anymore (e.g., the right panels in Fig. B.13). We here also assume a broadening parameter of $b=2 \mathrm{~km} \mathrm{~s}^{-1}$ as a conservative value, and find upper limits that are, depending on $\mathrm{S} / \mathrm{N}$, spectral coverage, and instrumental resolution, more or less constraining.

We note that we do not detect $\mathrm{H}_{2}$ in the 6 GRB-DLAs at $z>4$. However, the upper limits for most of them are not very constraining due to the strong Lyman- $\alpha$ forest. Especially for GRB 130606A, the forest is so strong that it is impossible to rule out that there is no absorption from molecular hydrogen. For this reason, the bursts at $z>4$ are labeled separately in some plots. Additionally, Lyman blanketing strongly affects the reliability of the measurements for the GRBs at reshift $z>4$. So far, $\mathrm{H}_{2}$ has 

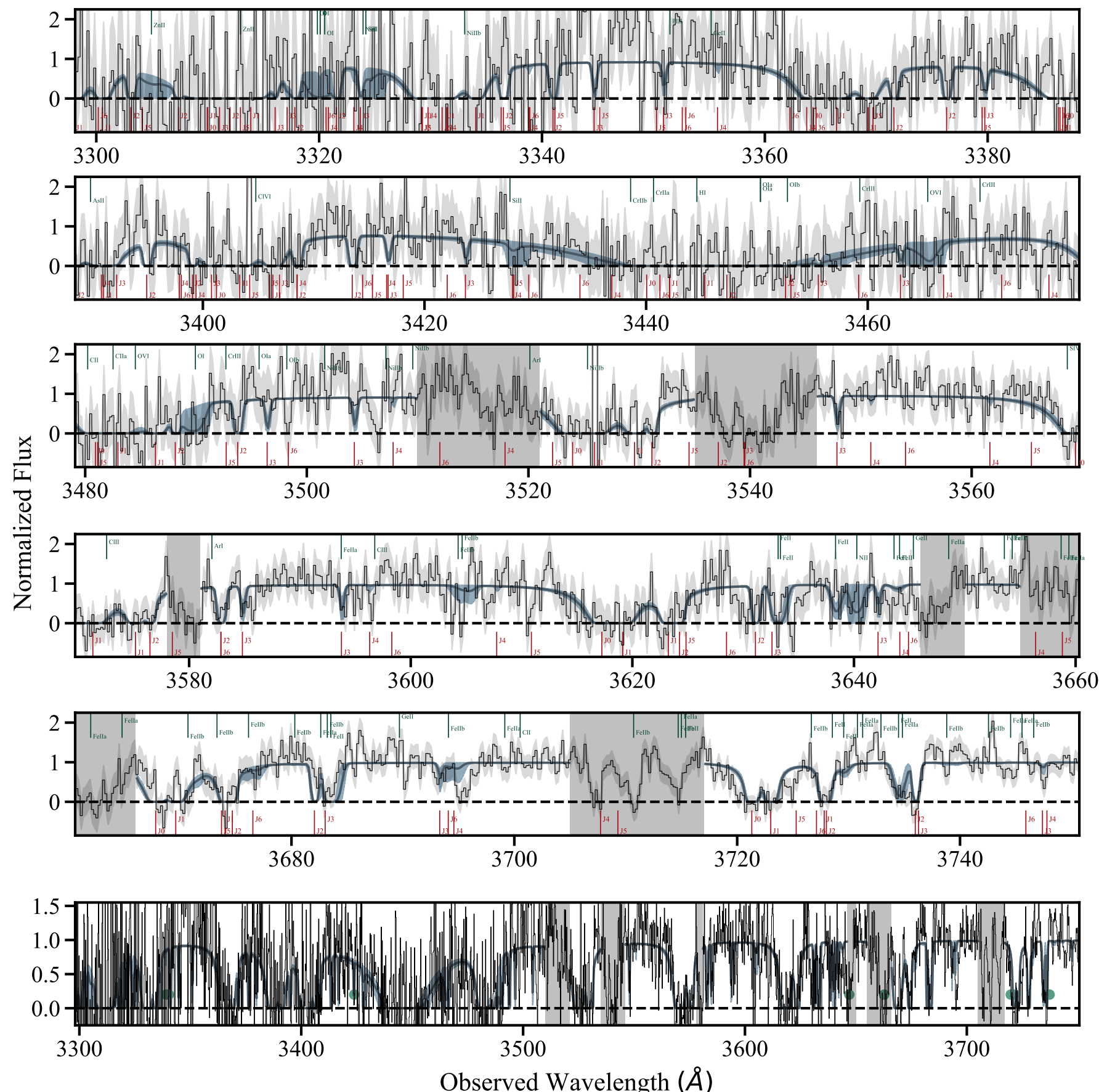

Fig. 9. X-shooter spectrum of GRB 120815A covering Lyman- and Werner-band absorption lines of molecular hydrogen. The best-FIT model is indicated by the solid blue lines and the corresponding 3- $\sigma$ confidence intervals by the blue shaded regions. Gray shaded regions were ignored during the FIT. The green circles in the bottom panel indicate the position of lines from the intervening systems at $z=1.539$ and 1.693 .

only been detected in one QSO-DLA at redshift $z>4$ (Ledoux et al. 2006). GRB 120909A is the $\mathrm{H}_{2}$-bearing GRB-DLA with the highest redshift $(z=3.929)$ found to date.

The results of our search for $\mathrm{H}_{2}$ are listed in Table A.2, which is divided into three parts: the six detections, the three tentative detections, and the rest of the bursts for which we do not find any convincing evidence for absorption from molecular hydrogen.

During our analysis, we found that some of the oscillator strength values for molecular hydrogen that we took from the compilation of Malec et al. (2010) are wrong, most likely due to a missing factor coming from the statistical weights ${ }^{7}$. We thus calculated the correct values following Morton (2003) and created a new data file which is available online ${ }^{8}$. While the values for the oscillator strength $f$ and the damping constant $\Gamma$, are still better than anything that can be currently measured, the wavelength or frequency positions are taken from the most recent lab experiments (Bailly et al. 2010, see their Tables 11 and 12).

\footnotetext{
7 See also the recently published paper by Ubachs et al. (2018).

8 https://doi.org/10.5281/zenodo. 1442558
} 

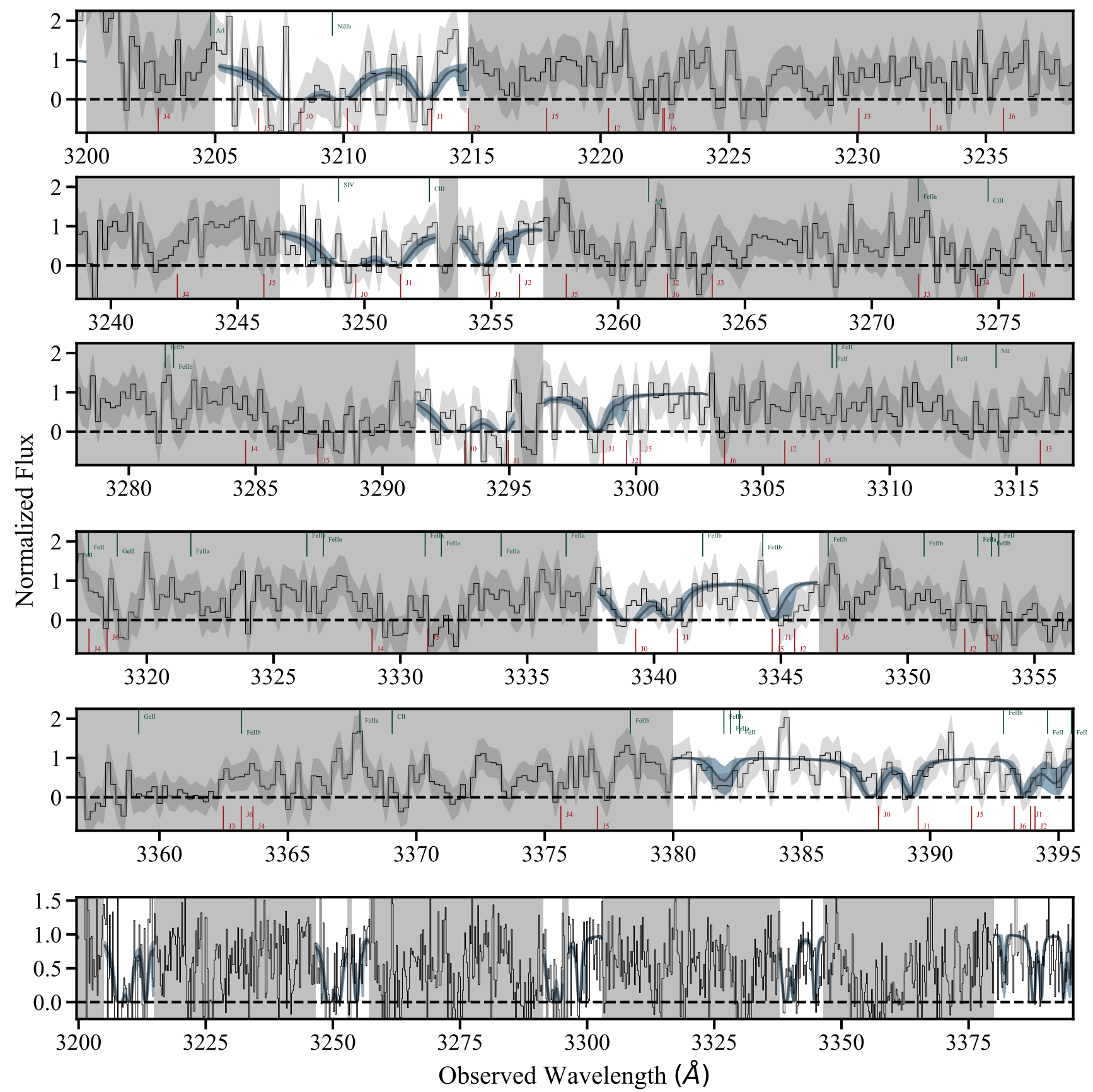

Fig. 10. X-shooter spectrum of GRB 150403A covering Lyman- and Werner-band absorption lines of molecular hydrogen. The best-FIT model is indicated by the solid blue line and the corresponding $3-\sigma$ confidence intervals by the blue shaded regions. Gray shaded regions were ignored during the FIT.

\subsection{Vibrationally excited molecular hydrogen}

The UV radiation from the GRB afterglow can theoretically pump any foreground $\mathrm{H}_{2}$ into its vibrationally excited levels that absorb at rest frame wavelengths $\lambda<1650 \AA$. For the six $\mathrm{H}_{2}$-bearing DLAs, as well as for the three tentative detections of $\mathrm{H}_{2}$ and the bursts at $z>4$, we thus additionally searched for vibrationally excited molecular hydrogen $\mathrm{H}_{2}^{*}$ by cross-correlating the observed spectra with the theoretical model from Draine (2000) and Draine \& Hao (2002), as was done before for GRB 120815A in Krühler et al. (2013) and for GRB 121024A in Friis et al. (2015) ${ }^{9}$. We confirm the detection in GRB 120815A

\footnotetext{
$9 \mathrm{H}_{2}^{*}$ was first detected by Sheffer et al. (2009) in GRB 080607
}

and also find tentative evidence for vibrationally excited molecular hydrogen in the spectrum of GRB 150403A (see Fig. 12). For GRB 121024A, we confirm the non-detection and we also find no evidence for $\mathrm{H}_{2}^{*}$ in 120327A, 120909A, 141109A, GRB 151021A, $160203 \mathrm{~A}$, and 170202A. In the case of the six GRB at $z>4$, we also find no convincing evidence for absorption from $\mathrm{H}_{2}^{*}$.

\subsection{Carbon monoxide}

We do not find evidence for carbon monoxide absorption lines in any of the 22 GRB spectra. $\mathrm{CO}$, which is primarily formed in the presence of $\mathrm{H}_{2}$, was previously detected in the $\mathrm{H}_{2}$ bearing DLA of GRB 080607 (Prochaska et al. 2009) and various $\mathrm{H}_{2}$-bearing QSO-DLAs (e.g., Srianand et al. 2008; 

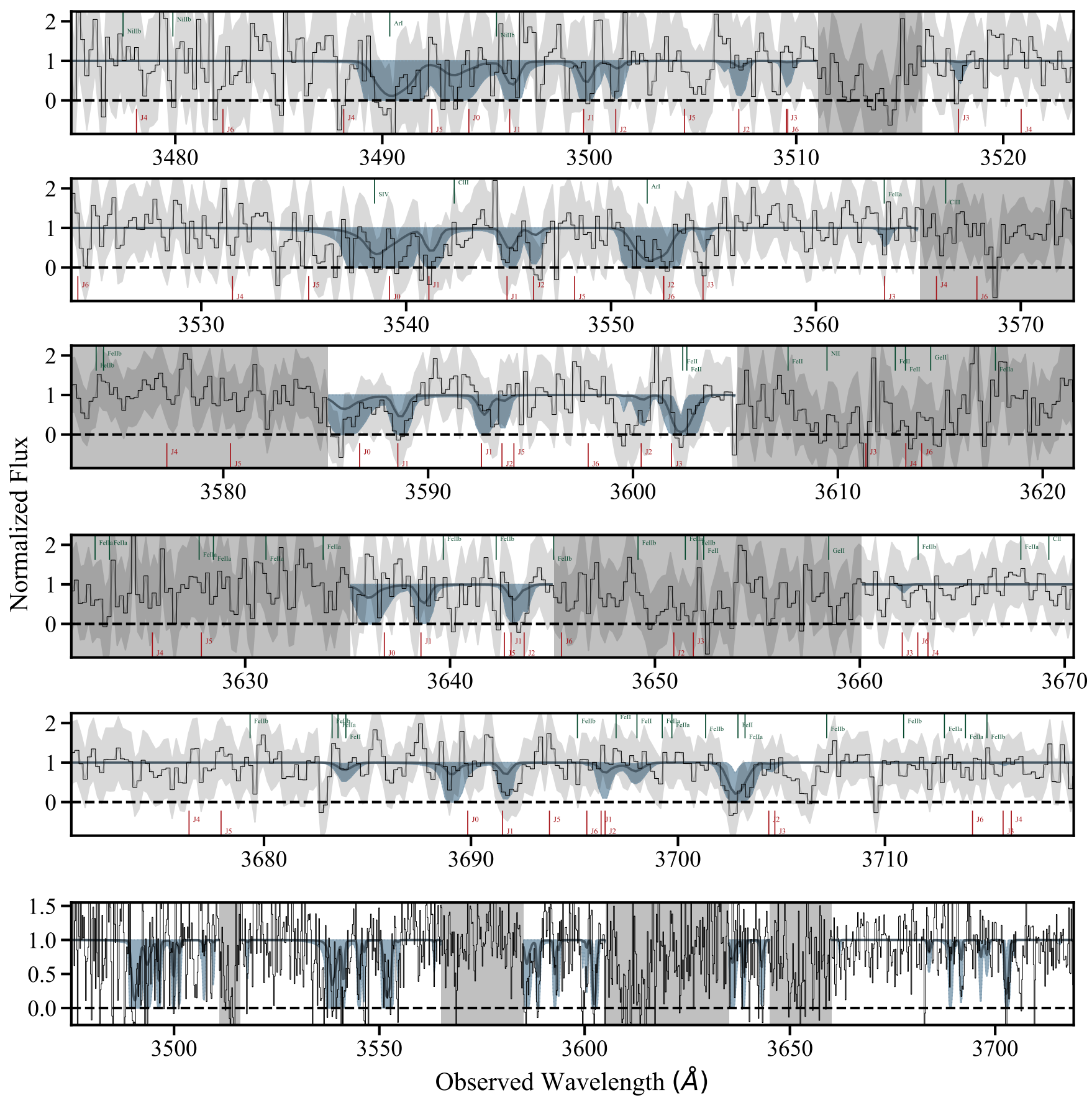

Fig. 11. X-shooter spectrum of GRB 151021A covering Lyman- and Werner-band absorption lines of molecular hydrogen. The best-FIT model is indicated by the solid blue lines and the corresponding $3-\sigma$ confidence intervals by the blue shaded regions. Gray shaded regions were ignored during the FIT.

Noterdaeme et al. 2010, 2018). However, the presence of molecular hydrogen seems not to be a sufficient condition for the presence of CO (Noterdaeme et al. 2015b). To determine upper limits for the $\mathrm{CO}$ column density for the six $\mathrm{H}_{2}$-bearing GRB-DLAs in our sample, we fitted the six strongest $\mathrm{CO}$ AX bandheads $(\mathrm{CO}$ $\mathrm{AX}(0-0)$ to $\mathrm{CO} \mathrm{AX}(5-0))$ simultaneously, under the conservative assumption that $b=2.0 \mathrm{~km} \mathrm{~s}^{-1}$. The resulting upper limits and also the corresponding upper limits for the $N(\mathrm{CO})$ to $N\left(\mathrm{H}_{2}\right)$ ratio are listed in Table 2. The upper limits on the $N(\mathrm{CO}) / N\left(\mathrm{H}_{2}\right)$ ratio range from $<10^{-2.1}$ to $<10^{-5.5}$ and are consistent with values found for diffuse molecular clouds, or translucent clouds, which are in the transition region between diffuse and dark
(Burgh et al. 2010). This is further supported by the relatively low extinction on the line of sight to the GRBs in our sample $\left(A_{V}(\right.$ depl. $\left.)<1.5 \mathrm{mag}\right)$.

\subsection{Overall results}

To summarize our results, we analysed X-shooter spectra of 22 GRB afterglows in order to derive the column densities of 10 different metal species as well as those of neutral atomic and molecular hydrogen in each GRB host galaxies line of sight (see Tables A.1 and A.2). By using these measurements we derive the elements relative abundances $[\mathrm{X} / \mathrm{H}]$ and further analyse their 
J. Bolmer et al.: Evidence for diffuse molecular gas and dust in the hearts of gamma-ray burst host galaxies

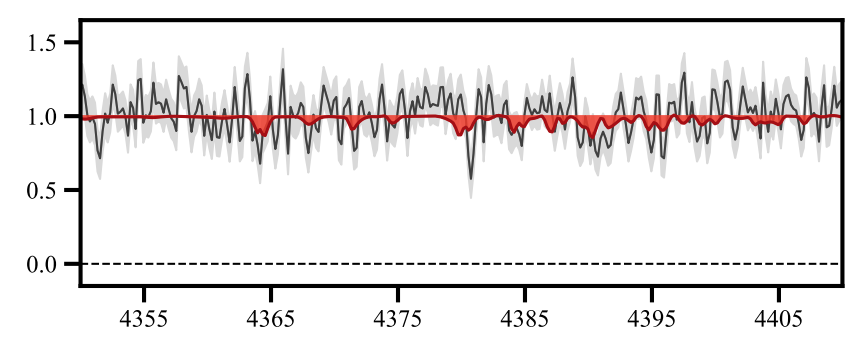

Table 2. Upper limits on the $\mathrm{CO}$ column density in the six $\mathrm{H}_{2}$ bearing GRB DLAs as well as the $N(\mathrm{CO}) / N\left(\mathrm{H}_{2}\right)$ ratios.

\begin{tabular}{ccc}
\hline \hline $\begin{array}{c}\text { GRB } \\
\text { yymmdd\# }\end{array}$ & $\begin{array}{c}\log N(\mathrm{CO}) \\
\mathrm{AX}(0-0) \text { to }(5-0)\end{array}$ & $N(\mathrm{CO}) / N\left(\mathrm{H}_{2}\right)$ \\
\hline $120327 \mathrm{~A}$ & $<15.3$ & $<10^{-2.1}$ \\
$120815 \mathrm{~A}$ & $<15.0$ & $<10^{-5.4}$ \\
$120909 \mathrm{~A}$ & $<14.2$ & $<10^{-3.2}$ \\
$121024 \mathrm{~A}$ & $<14.4$ & $<10^{-5.5}$ \\
$141109 \mathrm{~A}$ & $<15.9$ & $<10^{-2.1}$ \\
$150403 \mathrm{~A}$ & $<14.9$ & $<10^{-5.0}$ \\
\hline 080607 & $16.5 \pm 0.3$ & $\sim 10^{-4.7}$ \\
\hline
\end{tabular}

Notes. Also, listed are the values for GRB 080607 as taken from Prochaska et al. (2009). The CO upper limits $(3-\sigma)$ were determined assuming $b=2.0 \mathrm{~km} \mathrm{~s}^{-1}$ as a conservative value. In case of GRB 120327A, 120909A and 141109A, the $N(\mathrm{CO}) / N\left(\mathrm{H}_{2}\right)$ ratio was determined taking the $\mathrm{H}_{2}$ column densities measured for $b=2.0 \mathrm{~km} \mathrm{~s}^{-1}$ as given in Table A.2.

show pairwise scatter plots and histograms of the most important properties. By color and symbol, we differentiate between four different cases: (1) detections and (2) possible detections of absorption from molecular hydrogen as well as non-detections for (3) GRBs at redshift $z<4$ and the non-detections for (4) GRBs at redshift $z>4$.

The mean neutral hydrogen column density for the GRBDLAs in our sample is $\log \overline{N(\mathrm{HI})}=21.50 \pm 0.67$, and the individual values range from $\log N(\mathrm{HI})=19.88 \pm 0.01$ (GRB 130606, the only sub-DLA) to $\log N(\mathrm{H} \mathrm{I})=22.39 \pm 0.01$ for GRB 111008A, which is among the highest values observed to date (Prochaska et al. 2009; Sparre et al. 2014; Noterdaeme et al. 2015b). DLAs with such high column densities are expected to be very rare, because at $\log N(\mathrm{HI})>21.5$ the conversion from $\mathrm{H}$ I to $\mathrm{H}_{2}$ effectively steepens the column density distribution (Schaye 2001; Altay et al. 2011).

On average, the 22 GRB-DLAs have a metallicity of $\overline{[\mathrm{X} / \mathrm{H}]}=-1.27 \pm 0.37$, which is comparable to metallicities found for QSO-ESDLAs (Noterdaeme et al. 2015a). The average metallicity of the GRB-DLAs at $z>4$ is $\overline{[\mathrm{X} / \mathrm{H}]}=-1.39 \pm 0.42$, which is slightly lower than the mean metallicity for the bursts at redshift $2<z<4(\overline{[\mathrm{X} / \mathrm{H}]}=-1.23 \pm 0.37)$. This is in accordance with the results from Rafelski et al. (2012) and De Cia et al. (2018), that DLA metallicities decrease by a factor of 50-100 from redshift $z=0$ to $z=5$.

Interestingly we find that, while $\mathrm{H}_{2}$ is detected over almost the whole range of covered metallicities $[\mathrm{X} / \mathrm{H}]$, all detections and possible detections of molecular hydrogen are for DLAs with neutral hydrogen column densities larger than $\log N(\mathrm{HI})>$ 21.7. However, not all GRB-DLAs with $\log N(\mathrm{H} \mathrm{I})>21.7$ show absorption from $\mathrm{H}_{2}$. Also, as indicated by the dashed blue lines, which are the result of a Bayesian linear regression as described in Sect. 5, we find positive correlations for [Y/Fe] and $\log A_{V}$ (depl.) versus metallicity, as well as for $\mathcal{D T M}$ versus depletion [Y/Fe] and $\log A_{V}$ (depl.). A weak correlation is also found for $\mathcal{D T M}$ versus metallicity, which is, however, not as tight as the one determined by Wiseman et al. (2017b). Also, all $\mathrm{H}_{2}$-bearing systems have dust-to-metals ratios larger than $\mathcal{D T} \mathcal{M}>0.4$ and significant line-of-sight dust columns $\left(A_{V}\right.$ (depl.) $\left.>0.1 \mathrm{mag}\right)$. See also Fig. 7, which shows that for all $\mathrm{H}_{2}$-bearing GRB-DLAs as well as the tentative detections $A_{V}$ (depl.) is higher than $A_{V}$ (SED), indicating that the phase of the (high redshift) ISM that 


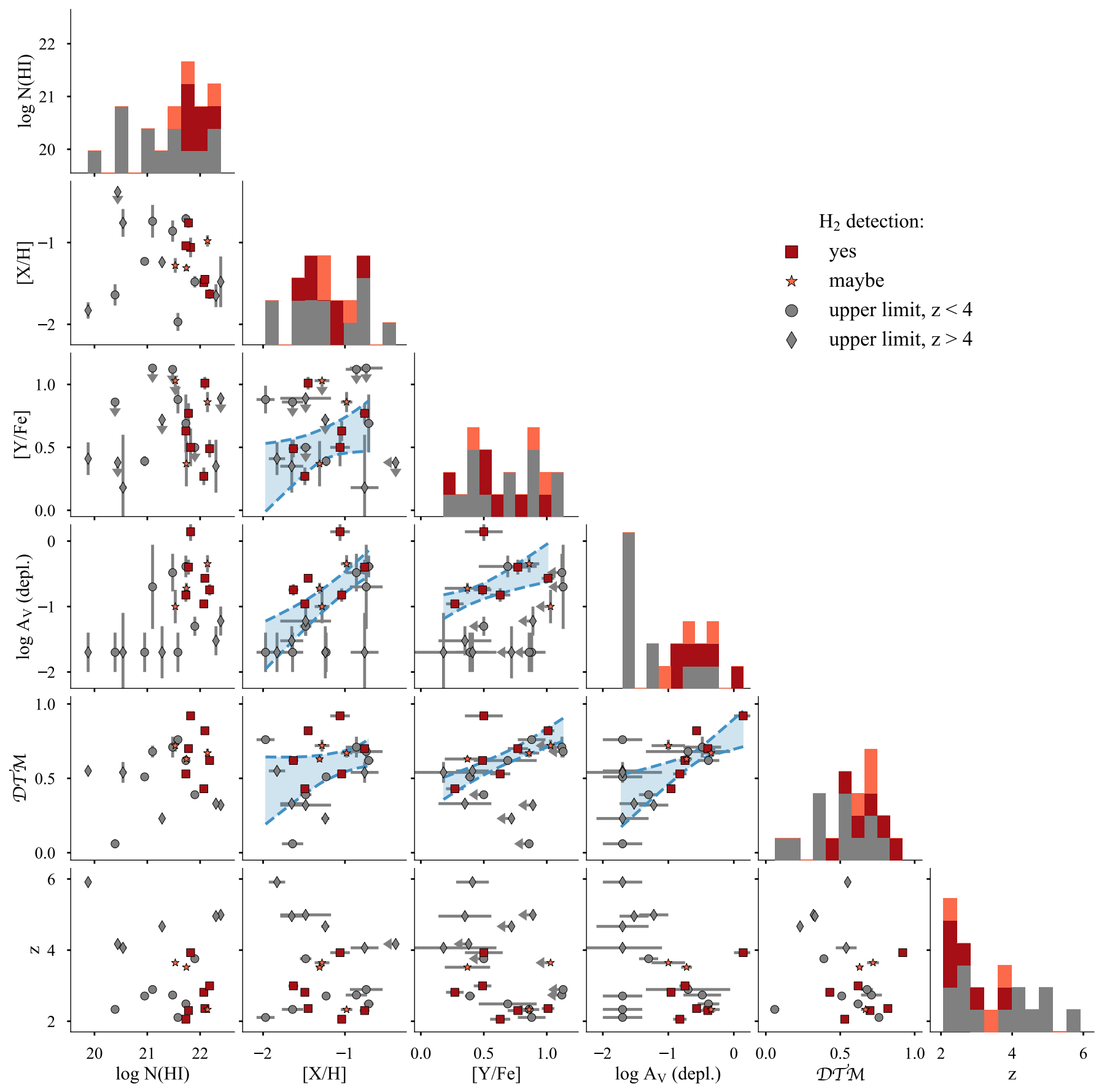

Fig. 13. Pairwise scatter plots and histograms of the most important properties of our sample of 22 GRB-DLAs at $z>2$. In case absorption from molecular hydrogen is detected, the data points are squares colored in red. Possible detections are indicated by orange stars, and upper limits are gray diamonds in case of DLAs at $z>4$ and circles otherwise. In blue, we show the results of a Baysian linear-regression analysis as described in Sect. 5.

is amenable to $\mathrm{H}_{2}$ formation might have a different dust composition or grain sizes. Furthermore, we do not find any evidence for absorption of $\mathrm{CO}$ in any of the spectra. And finally, we confirm the detection of vibrationally excited $\mathrm{H}_{2}^{*}$ in GRB 120815A and additionally find tentative evidence for $\mathrm{H}_{2}^{*}$ in GRB 150403A.

\section{Discussion}

The two main questions we seek to address in this paper are: (1) is there a lack of $\mathrm{H}_{2}$-bearing GRB-DLAs and, if so, why, and (2) how do GRB-DLAs compare to QSO-DLAs?

\section{1. $\mathrm{H}_{2}$ detection fraction in GRB-DLAs}

To answer the first question: there is no lack of $\mathrm{H}_{2}$-bearing GRB-DLAs. We here find evidence for absorption from $\mathrm{H}_{2}$ in $6 / 22(27 \%)$ of our GRB spectra, or even $9 / 22(41 \%)$ when including the tentative detections. When selecting GRB-DLAs at redshifts $2<z<4$ and with large neutral hydrogen column densities, $\log N(\mathrm{HI})>21.7$, the $\mathrm{H}_{2}$ detection fraction increases to $6 / 10(60 \%)$, or $8 / 10(80 \%)$, respectively. We want to stress that, although this is the first time a systematic search for $\mathrm{H}_{2}$ has been carried out in such a large sample of 22 GRB-DLAs, we are probably still biased against dusty sight-lines $\left(A_{V}>0.5 \mathrm{mag}\right)$. 
Since the production of molecular hydrogen is enabled by its formation onto dust grains, and because dust and $\mathrm{H}$ I provide shielding against $\mathrm{H}_{2}$ dissociating radiation, the true fraction of $\mathrm{H}_{2}$-bearing GRB-DLAs might be even higher than found here; also, because our upper limits on the $\mathrm{H}_{2}$ column density are not in all cases stringent, meaning that the relatively low spectral resolution of $\mathrm{X}$-shooter and sometimes a poor $\mathrm{S} / \mathrm{N}$ and/or a strong Lyman- $\alpha$ forest blanketing make it difficult to detect the narrow absorption lines from molecular hydrogen.

The lack of $\mathrm{H}_{2}$-bearing GRB-DLAs previously found by Ledoux et al. (2009) is consistent with our results. Indeed, from their sample of seven systems, five have neutral hydrogen column densities larger than $\log N(\mathrm{HI})>21.0$, and three have $\log N(\mathrm{HI})>21.7$. These three DLAs, however, have lower metallicities $(\overline{[\mathrm{X} / \mathrm{H}]}<-1.5)$ than the average of our 6 detections $(\overline{[\mathrm{X} / \mathrm{H}]}=-1.24 \pm 0.33)$, and also relatively low depletion factors, $[\mathrm{Y} / \mathrm{Fe}]<0.2$, compared to the average of our $\mathrm{H}_{2}$-bearing GRB-DLAs $(\overline{[\mathrm{Y} / \mathrm{Fe}]}=0.62 \pm 0.26)$. Since these 7 bursts were observed at high resolution using UVES, this sample is limited to the brightest and therefore least extinguished GRBs, which explains the aforementioned differences.

Since molecular gas has a small covering fraction, one can also use neutral carbon lines as tracers of cold gas and dust (Srianand et al. 2005; Ledoux et al. 2015). Heintz et al. (2019) systematically searched for absorption from $\mathrm{C}_{\mathrm{I}}$ in a sample of GRB-DLAs similar to ours. They find conclusive or tentative evidence for $\mathrm{C} I$ in the spectra of GRB 120815A, 121024A, and $150403 \mathrm{~A}$, which are three out of the six $\mathrm{H}_{2}$-bearing systems we found ${ }^{10}$. We note that $\mathrm{C}_{\mathrm{I}}$ is not detected in the $\mathrm{H}_{2}$-bearing system on the line of sight toward GRB 141109A and in neither of our three possible detections (151021A, 160203A, and 170202A). GRB 120909A did not enter their sample because the CI lines are blended with tellurics. Heintz et al. (2019) found a fraction of $25 \%$ of C I-detected GRB-DLAs, or $46 \%$ for DLAs with $\log N(\mathrm{HI})>21.7$. This is lower than our $\mathrm{H}_{2}$ detection fraction for systems with $\log N(\mathrm{HI})>21.7$, which is likely a consequence of the relatively low metallicities, which make it difficult to detect $\mathrm{C}$ I. For example, the DLA toward GRB $141109 \mathrm{~A}$ is, with $[\mathrm{X} / \mathrm{H}]$ approximately -1.6 , one of the most metal-poor absorbers in the sample.

Finally, that we do not find a lack of $\mathrm{H}_{2}$-bearing GRB-DLAs is also in agreement with the recent results from Arabsalmani et al. (2018), who report the detection of a molecular-gas-rich GRB host galaxy at $z=2.086$ based on the observation of CO emission lines. They showed that the GRB hosts with measured molecular gas mass are quite normal in terms of gas content when compared to the general population, and suggested that there is no sign of molecular gas deficiency, at variance with results based on low-redshift GRB hosts (Hatsukade et al. 2014; Stanway et al. 2015; Michałowski et al. 2016).

\subsection{Comparison of GRB- with QSO-DLA properties}

The fraction of $\mathrm{H}_{2}$-bearing systems in the general QSO-DLA population is only about $10 \%$ or less (Ledoux et al. 2003; Noterdaeme et al. 2008; Balashev et al. 2014; Jorgenson et al. 2014; Balashev \& Noterdaeme 2018). This is about three to

\footnotetext{
10 Heintz et al. (2019) also found C I in the spectra of GRB 120119A and 180325A. GRB 120119A is not part of our sample because of its lower redshift $(z \sim 1.73)$. GRB $180325 \mathrm{~A}$, at $z=2.248$, is not included in the present study either, because it is not in the complete sample presented in Selsing et al. (2019). In addition, its high visual extinction prevents the search for $\mathrm{H}_{2}$.
}

four times lower than what we found for GRB-DLAs. However, one should be cautious when comparing GRB- and QSO-DLA properties. In Fig. 14, we plot the metallicity, $[\mathrm{X} / \mathrm{H}]$, versus neutral hydrogen column density for the GRB-DLAs in our sample as well as for the QSO-DLAs from Noterdaeme et al. (2008; N08) and Noterdaeme et al. (2015a; N15). Additionally, we plot two QSO-ESDLAs from Balashev et al. (2017; B17) and Ranjan et al. (2018; R18). The histograms over metallicity for our GRB sample and for the QSO sample from N08 show that both GRB- and QSO-DLAs cover more or less the same range of metallicities, the only difference residing in the tail at lower metallicities which is covered by QSO-DLAs. However, looking at the histograms over $\log N(\mathrm{HI})$ reveals that we are likely dealing with different populations: GRBs generally tend to probe DLAs with higher neutral hydrogen column densities, $\log N(\mathrm{H} \mathrm{I})>21.5$, with an average of $\log \overline{N(\mathrm{HI})}=21.50 \pm 0.67$, while most QSO-DLAs have $\log N(\mathrm{H} \mathrm{I})<21.5$ and $\log \overline{N(\mathrm{H} \mathrm{I})}=$ $20.75 \pm 0.41$.

Concerning both the GRB- and QSO-DLAs bearing $\mathrm{H}_{2}$, all of them are found at metallicities $[\mathrm{X} / \mathrm{H}] \gtrsim-1.7$. However, only $\mathrm{H}_{2}$-bearing QSO-DLAs are found below $\log N(\mathrm{H} \mathrm{I})<21.7$. When considering DLAs at redshifts $2<z<4$ and with large neutral hydrogen column densities, $\log N(\mathrm{HI})>21.7$, the $\mathrm{H}_{2}$-detection fraction among QSO-DLAs is essentially the same as that of GRB-DLAs, that is 7/9 (78\%) of the QSO-DLAs bear $\mathrm{H}_{2}$ (Noterdaeme et al. 2015b,a). The majority of $\mathrm{H}_{2}$ bearing GRB- and QSO-DLAs is found at metal columns $\log N(\mathrm{X})>12.5$, and $\log N(\mathrm{X})>13.0$ for $\log N(\mathrm{H} \mathrm{I})>21.5$, as indicated in Fig. 14 by the dashed and dotted lines, respectively. This means that at higher H I column densities a lower metallicity is required to have the same metal column, and thus amount of dust, which needs a cold and dense environment but also a high cross-section of metals to form. Also, as previously shown by De Cia et al. (2016) and Wiseman et al. (2017b), we find that the $\mathcal{D T} \mathcal{M}$ increases with metallicity and that all $\mathrm{H}_{2}$ bearing GRB-DLAs have $\mathcal{D T} \mathcal{M}$ ratios higher than 0.4 , supporting the importance of grain growth for the production of dust and molecules.

A natural explanation for the higher fraction of $\mathrm{H}_{2}$-bearing systems in our 22 GRB-DLAs compared to the general population of QSO-DLAs, and the similar detection fractions at high $N(\mathrm{H} \mathrm{I})$ column densities, is the impact parameter (Arabsalmani et al. 2015). While higher H I and metal column densities are on average related to absorbing cold neutral gas within a galaxy, low column densities likely trace cool, circumgalactic environments (Pontzen et al. 2008; Prochaska et al. 2011; Noterdaeme et al. 2015b). As randomly distributed background sources, QSOs probe a wide range of impact parameters and therefore $\mathrm{H}$ I and metal column densities. GRBs, on the other hand, are usually found to originate from the central and/or brighter regions of their host galaxies (Fruchter et al. 2006; Lyman et al. 2017), where they are in most cases located behind relatively large column densities (Fynbo et al. 2009; Selsing et al. 2019).

Theoretical models predict that the $N(\mathrm{HI})$ threshold for the conversion from atomic to molecular hydrogen increases with decreasing metallicity (Savage et al. 1977; McKee \& Krumholz 2010; Sternberg et al. 2014). This is because systems with higher metallicities usually contain more dust (see Fig. 15), which provides shielding against UV radiation and enables the production of molecular hydrogen. This explains why the $\mathrm{H}_{2}$ bearing QSO-DLAs from the sample of N08 at $\log N(\mathrm{H} \mathrm{I}) \lesssim 21.0$ have relatively high metallicities $([\mathrm{X} / \mathrm{H}] \gtrsim-1.2$; with only one exception) and fall in a region that is barely probed by our 


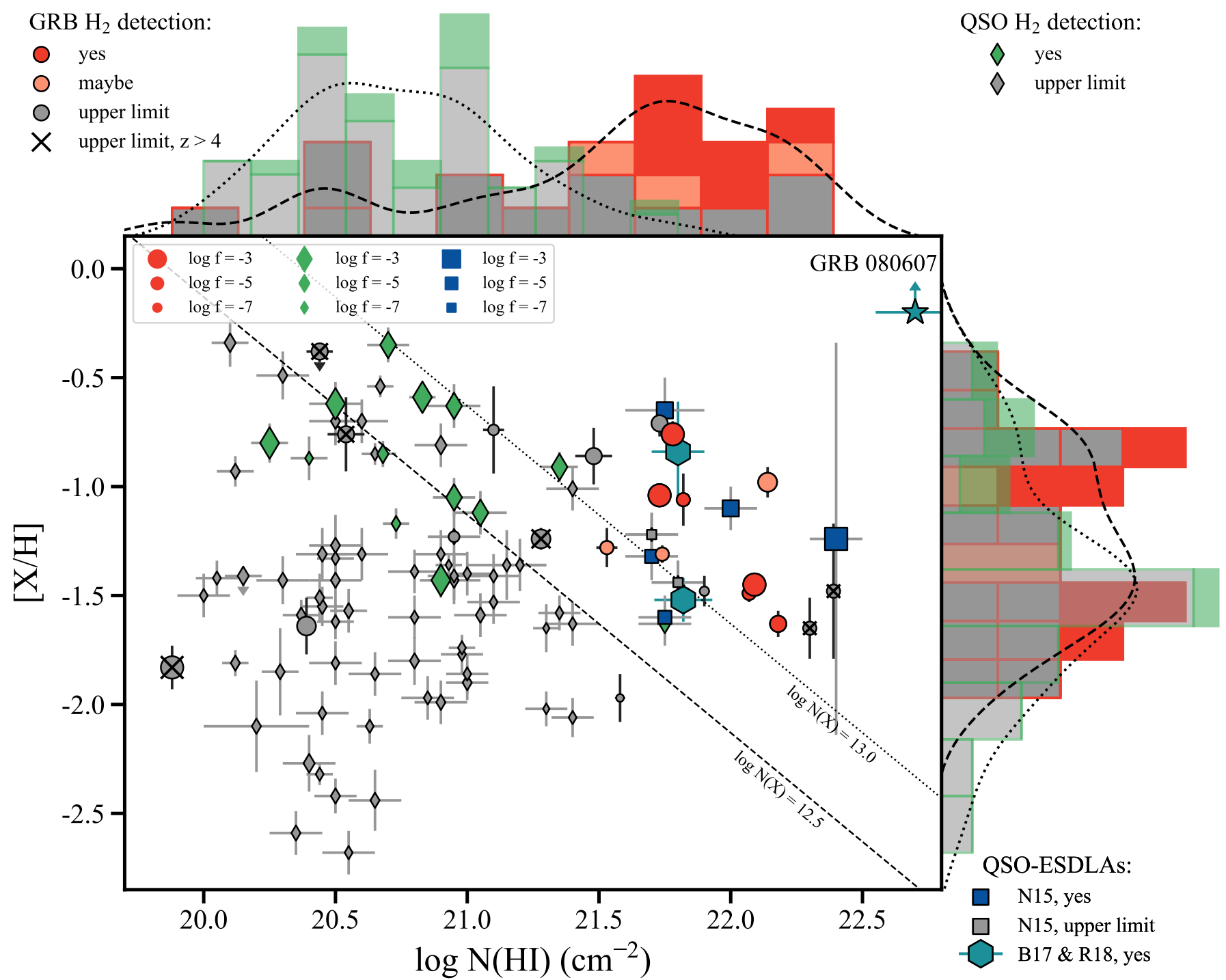

Fig. 14. Metallicity versus neutral atomic-hydrogen column density for the GRB-DLAs in our sample and the QSO-DLAs from Noterdaeme et al. (2008, 2015a; N15). Also shown are the two extremely strong QSO-DLAs from Balashev et al. (2017; B17) and Ranjan et al. (2018; B18). When molecular hydrogen is detected, the data points are colored (GRB-DLAs in red, QSO-DLAs in green and blue, as labeled). The symbol size represents the overall molecular fraction $\log f$, and in case of a non-detection the corresponding upper limit. The GRB-DLAs at $z>4$ are additionally marked with a cross. The dotted and dashed lines represent a constant metal column density of $\log N(\mathrm{X})=12.5$ and $\log N(\mathrm{X})=13.0$, respectively. Above and to the right of the scatter plot, we show the histograms and corresponding kernel density estimates for our GRB-DLAs and the N08 QSO-DLA sample. Systems with $\mathrm{H}_{2}$ detections are colored as labeled.

GRB-DLAs. Only two GRBs fall in that regime, 120712A and $151027 \mathrm{~B}$, which are both at redshift $z>4$ where $\mathrm{H}_{2}$ is hard to detect. Therefore, and also due to low number statistics in this regime, it is not surprising that we do not detect any $\mathrm{H}_{2}$-bearing GRB-DLAs with $\log N(\mathrm{HI})<21.0$. We note that for the intermediate regime with $21.0<\log N(\mathrm{H} \mathrm{I})<21.5$ and $[\mathrm{X} / \mathrm{H}]>-1.5$ the number statistics is very low for both the GRB- and QSODLAs and that it will be interesting to select a sample of DLAs in that region for future studies.

The impact parameter also has an effect on the metallicity distribution of DLAs, that is predicted to cluster between $[\mathrm{X} / \mathrm{H}]=-1.5$ and -1 (Cen 2012), which is true for our sample of 22 GRB-DLAs and also for the QSO-DLA sample from N08. The overall metallicity should floor at $[\mathrm{X} / \mathrm{H}]$ approximately -3 at $z=1.6-4$, which is what we see as a tail of low metallicities covered by QSO-DLAs. These systems with $[\mathrm{X} / \mathrm{H}]<-1.7$ are likely associated with the cold gas outside the galactic disk or halo, where the metallicity is low and little star formation takes place. GRBs, again, generally tend to probe the inner, starforming regions of their host galaxies, where the metallicity is expected to be higher $([\mathrm{X} / \mathrm{H}]>-1.7$; e.g. Belfiore et al. 2017; Sánchez-Menguiano et al. 2018).

As mentioned earlier, DLAs with higher metal columns usually contain more dust. This is evident from Fig. 15, which is similar to Fig. 14, but this time we plot the dust depletion factor, $[\mathrm{Y} / \mathrm{Fe}]$, versus metal column density $\log N(\mathrm{HI})+[\mathrm{X} / \mathrm{H}]$. One can see that $\mathrm{H}_{2}$-bearing DLAs are exclusively found at $[\mathrm{Y} / \mathrm{Fe}] \gtrsim 0.2$ and $\log N(\mathrm{HI})+[\mathrm{X} / \mathrm{H}] \gtrsim 19.5$. Also, the molecular fraction $f$ and the fraction of $\mathrm{H}_{2}$-bearing DLAs increase with the dust depletion factor and metal column density. At metal column densities larger than $\log N(\mathrm{H} \mathrm{I})+[\mathrm{X} / \mathrm{H}]>20.0$ and redshifts $2<z<4$, we detect $\mathrm{H}_{2}$ in six of $13(46 \%)$ systems, or nine of $13(69 \%)$ systems when including the tentative detections. For the QSO-DLAs from N08, this fraction is 4/9 (44\%) and for the 


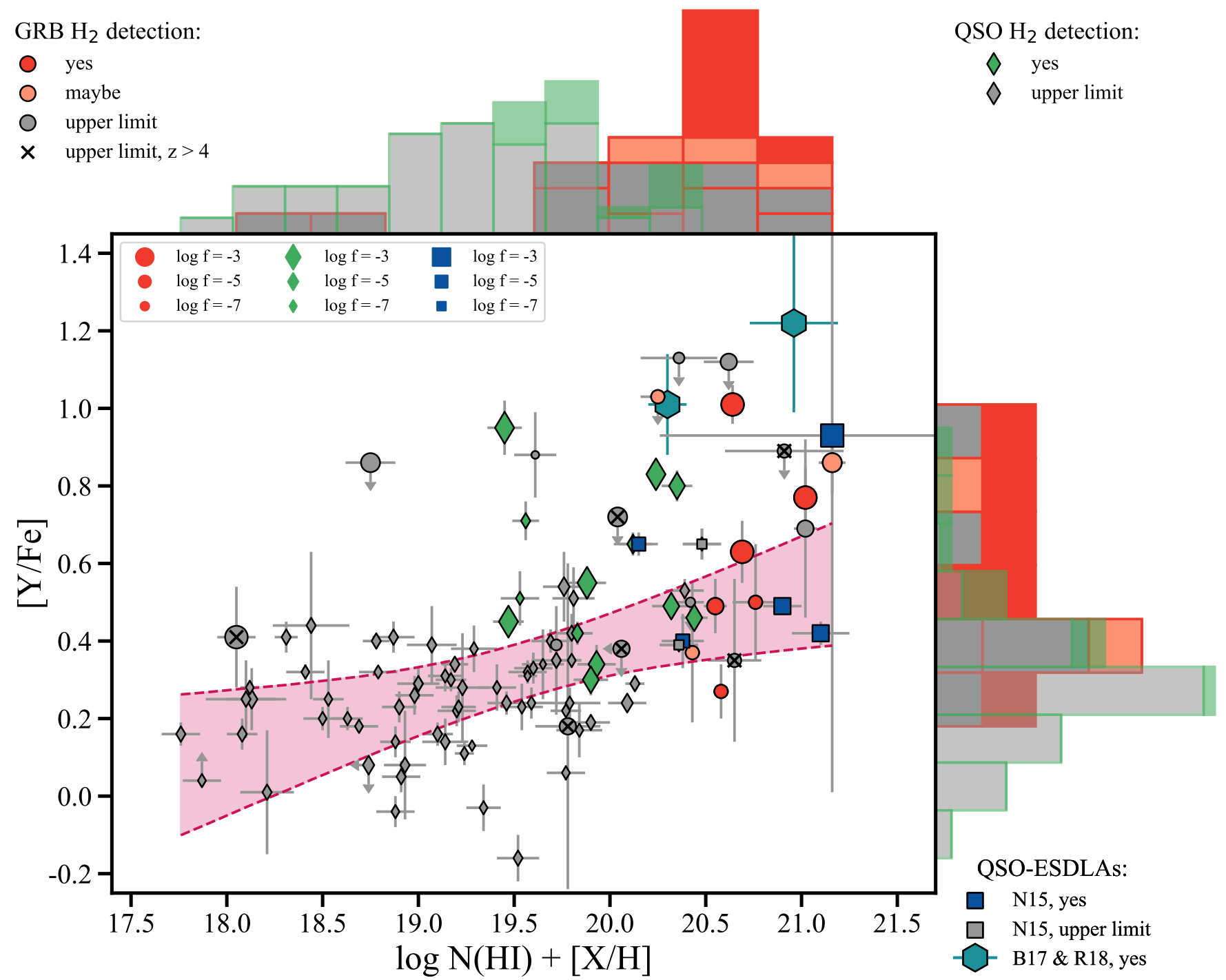

Fig. 15. Depletion factor, $[\mathrm{Y} / \mathrm{Fe}]$, versus metal column density, $\log N(\mathrm{HI})+[\mathrm{X} / \mathrm{H}]$, for the GRB-DLAs in our sample and the QSO-DLAs from Noterdaeme et al. (2008, 2015a; N15). Also shown are the two QSO-ESDLAs from Balashev et al. (2017; B17) and Ranjan et al. (2018; B18). When molecular hydrogen is detected, the data points are colored (GRB-DLAs in red, QSO-DLAs in green and blue, as labeled). The symbol size represents the overall molecular fraction $\log f$, and in case of a non-detection the corresponding upper limit. The GRB-DLAs at $z>4$ are additionally marked with a cross. The solid and dashed lines indicate the best-FIT linear model and corresponding $3 \sigma$ errors. Above and to the right of the scatter plot, we also show the histograms for our GRB-DLAs and the N08 QSO-DLA sample. Again, systems with $\mathrm{H}_{2}$ detections are colored as labeled. Upper limits are not included in the histograms.

QSO-ESDLAs from N15 it is 5/7 (71\%). So, also when selecting GRB- and QSO-DLAs based on metal column densities, the fraction of $\mathrm{H}_{2}$-bearing systems are similar.

Additionally, looking at the data points from both QSO- and GRB-DLAs, one can see that the dust depletion increases with increasing metal column density. The red line indicates the best linear FIT and the dashed lines the corresponding 3- $\sigma$ confidence intervals. The FIT was performed with a Bayesian linear regression and the posteriors on slope and intercept were computed using PyMC. To account for outliers, we use a Cauchy distribution as likelihood function. For the slope and intercept, we use normal priors centered on the best estimates from a simple first FIT using scipy.stats. linregress. Upper limits were not included in the FIT. It is interesting to note that almost all the DLAs that fall as outliers above this trend are strong $\mathrm{H}_{2}$-bearing systems. This is likely a result of the dust production that is enabled and the shielding provided by dust in those systems, which protects $\mathrm{H}_{2}$ from being photo-dissociated by LymanWerner photons. Therefore, most DLAs that follow the trend supposedly trace diffuse gas in the intercloud medium, whereas the outliers above more likely indicate diffuse molecular-rich gas.

\subsection{Nucleosynthesis signatures in the ISM}

The gas-phase metal abundances $[\mathrm{X} / \mathrm{H}]$ that we observe in GRB-DLAs do not directly represent the metallicity of the absorbing systems, because of dust depletion. Indeed dust depletion can dramatically lower the observed abundances, such as that it must be properly taken into account to be able to recover the real (dust-corrected) metallicity, $[\mathrm{M} / \mathrm{H}]$, and any nucleosynthetic signatures in the ISM. In Sect. 4.2, we FIT the observed abundances of several metals with the depletion patterns found in the Galaxy and QSO-DLAs by De Cia et al. (2016), and 
following Wiseman et al. (2017b). Any deviations from this FIT could in principle indicate peculiar abundances, for example, those produced by specific nucleosynthetic processes, such as $\alpha$-element enhancement. As shown in Fig. 6, the FIT to the depletion pattern is overall very good for most GRBs, with little deviations from QSO-DLAs. This is remarkable, given the different star-formation histories of galaxy counterparts of GRB- and QSO-DLAs. In particular, because of the higher starformation rates of GRB host galaxies (e.g., Krühler et al. 2015), $\alpha$-element enhancement could be expected. We only observe a tentative case of $\alpha$-element enhancement for GRB 121024A, with a Si overabundance of $\sim 0.5 \mathrm{dex}$, but no confirmation from other $\alpha$-elements such as $\mathrm{O}, \mathrm{Mg}$, Ti, nor $\mathrm{S}$. On the other hand, there is a tentative $\sim 0.2$ overabundance of the Fe-group elements $\mathrm{Fe}$ and $\mathrm{Cr}$, and the somewhat related $\mathrm{Zn}$, but with no apparent overabundance of $\mathrm{Ni}$, albeit the large error bar. Titanium is unfortunately constrained in only a few cases, because of the weakness of the Ti II absorption lines. Nevertheless, for two cases, namely GRB 120327A and GRB 161023A the observed Ti columns seem significantly lower than what can be expected from the depletion patterns. In both cases, no deviations of the other $\alpha$ elements are observed. One possibility is that this is indeed a peculiar underabundance of Ti. Alternatively, and perhaps more likely, this may signal that the values for $\mathrm{Ti}$ in the depletion patterns may be imprecise. Indeed, the depletion sequences were not characterized for DLAs for $\mathrm{Ti}$ and $\mathrm{Ni}$, but instead their slope was extrapolated to low-metallicity systems by De Cia et al. (2016) from the Galactic values of Jenkins (2009). This might have caused a somewhat shallower slope of the depletion sequence of $\mathrm{Ti}$, and thus a significant effect in the Ti depletion pattern, because Ti depletes very heavily. Quantifying this possible effect requires analyzing a large sample of QSO-DLAs and is beyond the scope of this paper.

\subsection{The lack of carbon monoxide}

The lack of carbon monoxide could be related to the low metallicity and/or the relatively low dust content $\left(A_{V}(\right.$ depl. $)<$ $1.5 \mathrm{mag}$ ) of the systems, meaning that not enough oxygen and carbon are present to form $\mathrm{CO}$, or not enough shielding against the photodissociating radiation is provided (Bolatto et al. 2013; Glover \& Clark 2016; Balashev et al. 2017). Alternatively, the lack of $\mathrm{CO}$ absorption could be the result of a geometrical effect, because $\mathrm{CO}$ should only reside in the inner core of the molecular cloud, where more shielding is provided. In this scenario, the GRB line-of-sight would cross through the outer edge of the cloud where only $\mathrm{H}_{2}$ is present. In Fig. 16, we plot the $\mathrm{CO}$ versus the $\mathrm{H}_{2}$ column densities for GRB and QSO-DLAs as well as for Galactic sight-lines. All our upper limits on the $\mathrm{CO}$ column density are consistent with the relation found by Burgh et al. (2010) for diffuse and translucent clouds in the Milky Way.

\subsection{Comparison with Galactic sight-lines}

In general, a comparison with Galactic sight-lines is complicated, due to the fact that QSO and GRB-DLAs do not have metallicities close to solar, but rather relatively low metallicities on the order of one tenth of solar. Also, the dust-to-metal ratios are usally lower than in the Galaxy (De Cia et al. 2013, 2016; Wiseman et al. 2017b), so that even for the same amount of metals, one would expect less dust and thus less molecules. Nevertheless, in Fig. 17, we plot the molecular fraction $f$ versus

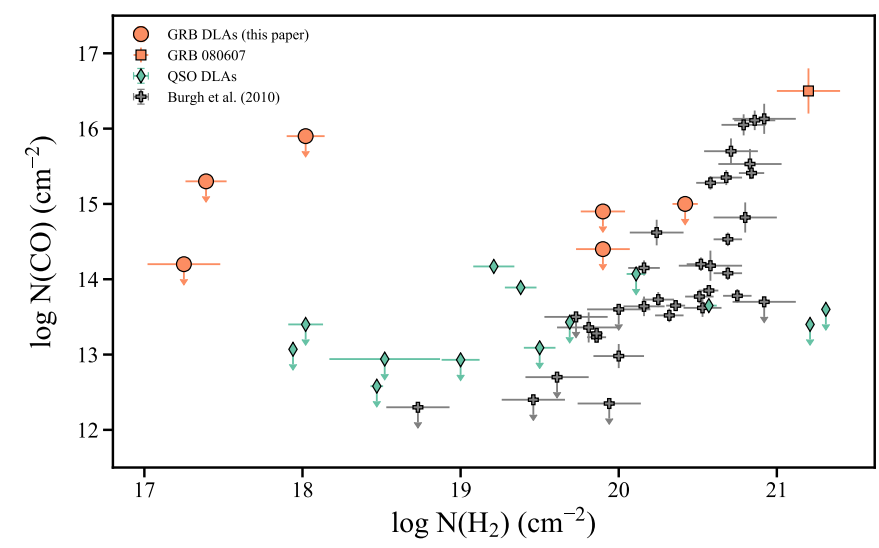

Fig. 16. $\mathrm{CO}$ versus $\mathrm{H}_{2}$ column densities for the $\mathrm{H}_{2}$-bearing GRB-DLAs presented in this paper compared to the $\mathrm{H}_{2}$-bearing QSO-DLAs from Balashev et al. (2017); Noterdaeme et al. (2018); Ranjan et al. (2018). Additionally, we plot the results from Burgh et al. (2010) for sight-lines through diffuse and translucent clouds in the Galaxy and the result from Prochaska et al. (2009) for the DLA toward GRB 080607.

the molecular hydrogen column density for GRB and QSODLAs in comparison to Galactic sight-lines. One can see that only about half of the $\mathrm{H}_{2}$-bearing DLAs with $\log N(\mathrm{HI})>21.7$ have molecular fractions consistent with the galactic diffuse and translucent clouds from Burgh et al. (2010). For the rest of the DLAs, the molecular fraction is much lower, which is also the case for the upper limits we could put on the non-detections. This could be a result of the generally low metallicities and low dust columns.

\subsection{Distances and nature of the absorbing clouds}

To determine the distance of the absorbing cloud to the GRB explosion site, it is necessary to observe absorption-line variability to trace the electronic populations in different energy levels. Such an analysis has only been possible for a handful of cases and the distances were all found to be 50 to more than several hundred parsecs (Vreeswijk et al. 2007, 2011; Ledoux et al. 2009; D'Elia et al. 2009; Hartoog et al. 2013). Alternatively, we could use the $\mathrm{H}_{2}^{*}$ detections and non-detections of our six $\mathrm{H}_{2}$-bearing GRB-DLAs, to determine the required UV flux to populate the vibrationally-excited levels. This flux could be compared with the actual UV flux measured for the afterglow light curve in order to put a constraint or a lower limit to the distance of the absorbing cloud. Nevertheless, this exercise is beyond the scope of this paper, and we just note that the detection of molecular hydrogen alone, should imply a distance of at least $\gtrsim 0.5-1 \mathrm{kpc}$ between the GRB and the absorbing gas to avoid photo-ionization. For example, Ledoux et al. (2009) find for GRB 050730 that $\mathrm{H}_{2}$ photo-dissociation can only be effective in clouds with distances smaller than $d<500$ pc (see their Fig. 7).

Additionally, we detect the fine-structure levels of $\mathrm{O}$ I, O I* and $\mathrm{OI}^{* *}$, in almost all of the GRB spectra (see Fig. 4). These lines are commonly detected in GRB-DLAs and indicative of high densities and temperatures, and therefore add to the picture of the absorbing gas having mixed phases and being under the influence of a local radiation field, i.e., diffuse molecular clouds.

Our results are thus pointing to a picture where GRB and QSO-DLAs with very high neutral column densities $(\log N(\mathrm{HI})>21.7)$ or metal column densities $(\log N(\mathrm{HI})+$ $[\mathrm{X} / \mathrm{H}]>20)$ are associated with sight-lines passing close to the galactic center, where the gas pressure is higher 


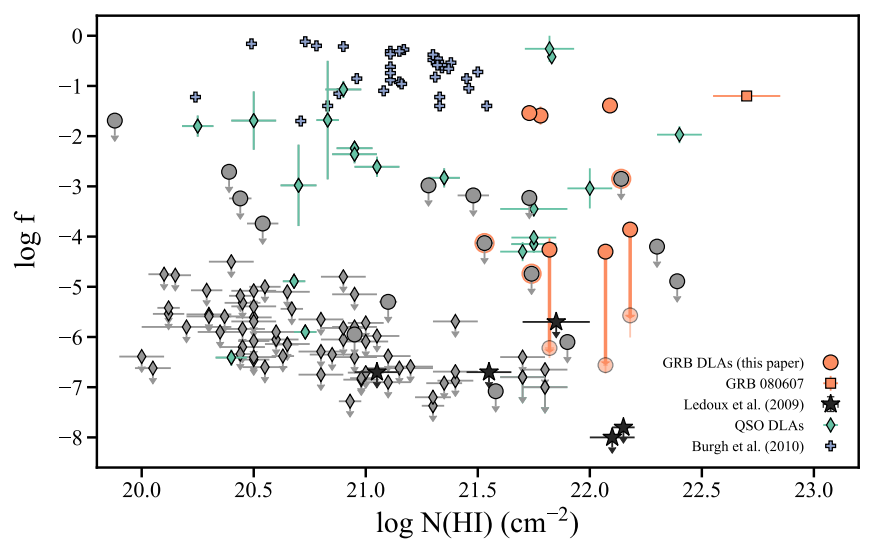

Fig. 17. Molecular fraction versus neutral atomic-hydrogen column density for the GRB-DLAs from this paper and Ledoux et al. (2009) and Prochaska et al. (2009) compared to QSO-DLAs (Noterdaeme et al. 2008, 2015a; Balashev et al. 2017; Ranjan et al. 2018) and Galactic sight-lines probing diffuse and translucent clouds (Burgh et al. 2010). $\mathrm{H}_{2}$-bearing DLAs are plotted with colored symbols and the nondetections in gray. For GRB 120327A, 120909A, and 141109A, we plot the molecular fraction for both $b=2$ and $b=10 \mathrm{~km} \mathrm{~s}^{-1}$.

(Blitz \& Rosolowsky 2006; Balashev et al. 2017), the conversion of $\mathrm{HI}$ to $\mathrm{H}_{2}$ steepens the column density distribution (Altay et al. 2011; Noterdaeme et al. 2014), and thus a higher fraction of $\mathrm{H}_{2}$-bearing systems is expected. And, since the absorbing gas is likely located at distances of several hundred parsec from the $\mathrm{GRB}$, the $\mathrm{H}_{2}$-bearing GRB-DLAs are not associated with the star-forming regions where the GRB progenitors are born, but with diffuse molecular clouds within the host galaxy.

\section{Summary}

For the first time, we were able to use a large, less-biased sample of 22 GRBs, to perform a systematic search for molecular hydrogen in DLAs associated with GRB host galaxies, and to study the effects of metallicity and dust depletion in the diffuse interstellar medium of these galaxies at high redshift. The main results derived from our analysis can be summarized as follows:

(i) There is no lack of $\mathrm{H}_{2}$ in GRB-DLAs. We find evidence for absorption from molecular hydrogen in 6 out of 22 such systems and claim three additional tentative detections. This constitutes a fraction of $27 \%(41 \%)$, which is three to four times larger than in the general population of QSO-DLAs $(\leq 10 \%)$, but comparable to the fraction of $\mathrm{H}_{2}$-bearing QSODLAs that cover the same range of neutral hydrogen column densities and redshifts, the so-called extremely-strong QSODLAs (ES-DLAs). Also, all $\mathrm{H}_{2}$-bearing GRB-DLAs are found to be associated with significant dust extinction, $A_{V}>$ $0.1 \mathrm{mag}$, and have dust-to-metals ratios $\mathcal{D T} \mathcal{M}>0.4$, confirming the importance of dust grains for the production of molecules.

(ii) Both, QSO- and GRB-DLAs, with column densities $\log N(\mathrm{HI})>21.7$ at redshifts $2<z<4$ appear to probe similar systems, that is sight-lines with small impact parameters that are passing close to the galactic central regions, where the gas pressure is higher and the transition of $\mathrm{HI}$ to $\mathrm{H}_{2}$ is facilitated. In this range, the fraction of $\mathrm{H}_{2}$-bearing systems is much higher and comparable in both QSO- and GRB-DLAs (60-80\%). These systems are likely diffuse molecular clouds, which in the case of GRBs, are unrelated to the star-forming region where the explosion occurred. (iii) At lower column densities $(\log N(\mathrm{H} \mathrm{I})<21.7)$, QSO-DLAs need on average a higher metallicity to contain significant amounts of molecular hydrogen. This can be explained naturally by the fact that systems with a higher metallicity contain on average more dust, which provides shielding and enables the production of $\mathrm{H}_{2}$. Our sample of GRB-DLAs does not significantly probe low hydrogen column densities (i.e., only $22 \%$ of the sample has $\log N(\mathrm{H} \mathrm{I})<21.0$ ) because GRBs are found to originate from or closer to the central regions of their host galaxies. On the other hand, as randomly distributed background sources, QSOs sample a wide range of impact parameters.

(iv) The actual fraction of $\mathrm{H}_{2}$-bearing GRB-DLAs might be higher than the one we found here because our sample is probably still biased against dusty sight-lines (no sight-line with $\left.A_{V}(\mathrm{SED})>0.5 \mathrm{mag}\right)$. More dust provides more shielding, adds more catalyst to aid $\mathrm{H}_{2}$ production, and indicates an advanced grain chemistry and thus a higher chance to detect molecules. Our upper limits on the $\mathrm{H}_{2}$ column density are also not in all cases stringent, meaning that the relatively low spectral resolution of $\mathrm{X}$-shooter and sometimes a poor $\mathrm{S} / \mathrm{N}$ and/or a crowded Lyman- $\alpha$ forest make it difficult to detect the narrow absorption lines of molecular hydrogen.

(v) In the future, it will be important to expand the surveys of GRBs with prompt optical spectroscopy in order to increase sample sizes. With a new class of $30 \mathrm{~m}$ telescopes like the ELT, we should also be able to detect GRB afterglows behind translucent $\left(1<A_{V}<5 \mathrm{mag}\right)$ and possibly also Giant Molecular Clouds $\left(A_{V}>5 \mathrm{mag}\right)$. This will allow to study the physical conditions in dense molecular gas at high redshift.

Acknowledgements. The lead author acknowledges support from a studentship at the European Southern Observatory in Chile and thanks the many astronomers who dedicated their time observing the numerous GRBs with VLT/X-shooter. P.S. acknowledges support through the Sofja Kovalevskaja Award from the Alexander von Humboldt Foundation of Germany, and J.B. acknowledges support through this award. K.E.H. and P.J. acknowledge support by a Project Grant (162948-051) from The Icelandic Research Fund. J.J. acknowledges support from NOVA and NWO-FAPESP grant for advanced instrumentation in astronomy. Finally, we are indebted to Thomas Krühler for providing us with a code to generate synthetic GRB afterglow spectra.

\section{References}

Altay, G., Theuns, T., Schaye, J., Crighton, N. H. M., \& Dalla Vecchia, C. 2011, ApJ, 737, L37

Arabsalmani, M., Møller, P., Fynbo, J. P. U., et al. 2015, MNRAS, 446, 990

Arabsalmani, M., Le Floc'h, E., Dannerbauer, H., et al. 2018, MNRAS, 476, 2332

Asplund, M., Grevesse, N., Sauval, A. J., \& Scott, P. 2009, ARA\&A, 47, 481

Bailly, D., Salumbides, E., Vervloet, M., \& W. Ubachs. 2010, Mol. Phys., 108, 827

Balashev, S. A., \& Noterdaeme, P. 2018, MNRAS, 478, L7

Balashev, S. A., Klimenko, V. V., Ivanchik, A. V., et al. 2014, MNRAS, 440, 225 Balashev, S. A., Noterdaeme, P., Rahmani, H., et al. 2017, MNRAS, 470, 2890 Belfiore, F., Maiolino, R., Tremonti, C., et al. 2017, MNRAS, 469, 151

Betancourt, M. 2017, ArXiv e-prints [arXiv:1706.01520]

Bigiel, F., Leroy, A., Walter, F., et al. 2008, AJ, 136, 2846

Bigiel, F., Leroy, A. K., Walter, F., et al. 2011, ApJ, 730, L13

Blitz, L., \& Rosolowsky, E. 2006, ApJ, 650, 933

Bolatto, A. D., Wolfire, M., \& Leroy, A. K. 2013, ARA\&A, 51, 207

Bolmer, J., Greiner, J., Krühler, T., et al. 2018, A\&A, 609, A62

Burgh, E. B., France, K., \& Jenkins, E. B. 2010, ApJ, 708, 334

Cen, R. 2012, ApJ, 748, 121

Christensen, L., Fynbo, J. P. U., Prochaska, J. X., et al. 2011, ApJ, 727, 73

Covino, S., Melandri, A., Salvaterra, R., et al. 2013, MNRAS, 432, 1231

De Cia, A. 2018, A\&A, 613, L2

De Cia, A., Ledoux, C., Savaglio, S., Schady, P., \& Vreeswijk, P. M. 2013, A\&A, 560, A 88 
De Cia, A., Ledoux, C., Mattsson, L., et al. 2016, A\&A, 596, A97

De Cia, A., Ledoux, C., Petitjean, P., \& Savaglio, S. 2018, A\&A, 611, A76

de Ugarte Postigo, A., Thöne, C. C., Bolmer, J., et al. 2018, A\&A, 620, A119

D'Elia, V., Fiore, F., Perna, R., et al. 2009, ApJ, 694, 332

D’Elia, V., Fynbo, J. P. U., Covino, S., et al. 2010, A\&A, 523, A36

D’Elia, V., Fynbo, J. P. U., Goldoni, P., et al. 2014, A\&A, 564, A38

Draine, B. T. 2000, ApJ, 532, 273

Draine, B. T., \& Hao, L. 2002, ApJ, 569, 780

Ellison, S. L., Vreeswijk, P., Ledoux, C., et al. 2006, MNRAS, 372, L38

Fox, A. J., Ledoux, C., Vreeswijk, P. M., Smette, A., \& Jaunsen, A. O. 2008, A\&A, 491, 189

Friis, M., De Cia, A., Krühler, T., et al. 2015, MNRAS, 451, 167

Fruchter, A. S., Levan, A. J., Strolger, L., et al. 2006, Nature, 441, 463

Fynbo, J. P. U., Starling, R. L. C., Ledoux, C., et al. 2006, A\&A, 451, L47

Fynbo, J. P. U., Jakobsson, P., Prochaska, J. X., et al. 2009, ApJS, 185, 526

Fynbo, J. P. U., Krühler, T., Leighly, K., et al. 2014, A\&A, 572, A12

Glover, S. C. O., \& Clark, P. C. 2016, MNRAS, 456, 3596

Gong, M., Ostriker, E. C., \& Kim, C.-G. 2018, ApJ, 858, 16

Greiner, J., Krühler, T., Klose, S., et al. 2011, A\&A, 526, A30

Hartoog, O. E., Wiersema, K., Vreeswijk, P. M., et al. 2013, MNRAS, 430, 2739

Hartoog, O. E., Malesani, D., Fynbo, J. P. U., et al. 2015, A\&A, 580, A139

Hatsukade, B., Ohta, K., Endo, A., et al. 2014, Nature, 510, 247

Heintz, K. E., Watson, D., Jakobsson, P., et al. 2018, MNRAS, 1393, 1402

Heintz, K. E., Ledoux, C., Fynbo, J. P. U., et al. 2019, A\&A, 621, A20

Jakobsson, P., Fynbo, J. P. U., Ledoux, C., et al. 2006, A\&A, 460, L13

Jenkins, E. B. 2009, ApJ, 700, 1299

Jorgenson, R. A., Murphy, M. T., Thompson, R., \& Carswell, R. F. 2014, MNRAS, 443, 2783

Kanekar, N., Braun, R., \& Roy, N. 2011, ApJ, 737, L33

Kennicutt, R. C., \& Evans, N. J. 2012, ARA\&A, 50, 531

Kim, T. S., Carswell, R. F., Cristiani, S., D’Odorico, S., \& Giallongo, E. 2002, MNRAS, 335, 555

Kisielius, R., Kulkarni, V. P., Ferland, G. J., Bogdanovich, P., \& Lykins, M. L. 2014, ApJ, 780, 76

Kisielius, R., Kulkarni, V. P., Ferland, G. J., et al. 2015, ApJ, 804, 76

Krühler, T., Ledoux, C., Fynbo, J. P. U., et al. 2013, A\&A, 557, A18

Krühler, T., Malesani, D., Fynbo, J. P. U., et al. 2015, A\&A, 581, A125

Krumholz, M. R., McKee, C. F., \& Tumlinson, J. 2009, ApJ, 693, 216

Krumholz, M. R., Dekel, A., \& McKee, C. F. 2012, ApJ, 745, 69

Ledoux, C., Petitjean, P., \& Srianand, R. 2003, MNRAS, 346, 209

Ledoux, C., Petitjean, P., \& Srianand, R. 2006, ApJ, 640, L25

Ledoux, C., Vreeswijk, P. M., Smette, A., et al. 2009, A\&A, 506, 661

Ledoux, C., Noterdaeme, P., Petitjean, P., \& Srianand, R. 2015, A\&A, 580, A8

Leroy, A. K., Walter, F., Sandstrom, K., et al. 2013, AJ, 146, 19

Lodders, K., Palme, H., \& Gail, H. P. 2009, Landolt-Börnstein (Berlin: Springer), 4B, 44

Lyman, J. D., Levan, A. J., Tanvir, N. R., et al. 2017, MNRAS, 467, 1795

Malec, A. L., Buning, R., Murphy, M. T., et al. 2010, MNRAS, 403, 1541

McKee, C. F., \& Krumholz, M. R. 2010, ApJ, 709, 308

McKee, C. F., \& Ostriker, E. C. 2007, ARA\&A, 45, 565

Ménard, B., \& Fukugita, M. 2012, ApJ, 754, 116

Michałowski, M. J., Castro Cerón, J. M., Wardlow, J. L., et al. 2016, A\&A, 595, A72

Morton, D. C. 2003, ApJS, 149, 205

Noterdaeme, P., Ledoux, C., Petitjean, P., \& Srianand, R. 2008, A\&A, 481, 327

Noterdaeme, P., Petitjean, P., Ledoux, C., et al. 2010, A\&A, 523, A80

Noterdaeme, P., Petitjean, P., Pâris, I., et al. 2014, A\&A, 566, A24

Noterdaeme, P., Petitjean, P., \& Srianand, R. 2015a, A\&A, 578, L5

Noterdaeme, P., Srianand, R., Rahmani, H., et al. 2015b, A\&A, 577, A24

Noterdaeme, P., Ledoux, C., Zou, S., et al. 2018, A\&A, 612, A58

Perley, D. A., Morgan, A. N., Updike, A., et al. 2011, AJ, 141, 36

Pontzen, A., Governato, F., Pettini, M., et al. 2008, MNRAS, 390, 1349

Prochaska, J. X. 2006, ApJ, 650, 272

Prochaska, J. X., Chen, H.-W., \& Bloom, J. S. 2006, ApJ, 648, 95

Prochaska, J. X., Sheffer, Y., Perley, D. A., et al. 2009, ApJ, 691, L27

Prochaska, J. X., Weiner, B., Chen, H. W., Mulchaey, J., \& Cooksey, K. 2011, ApJ, 740, 91

Rafelski, M., Wolfe, A. M., Prochaska, J. X., Neeleman, M., \& Mendez, A. J. 2012, ApJ, 755, 89

Ranjan, A., Noterdaeme, P., Krogager, J. K., et al. 2018, A\&A, 618, A184

Sánchez-Menguiano, L., Sánchez, S. F., Pérez, I., et al. 2018, A\&A, 609, A119

Savage, B. D., \& Sembach, K. R. 1996, ApJ, 470, 893

Savage, B. D., Bohlin, R. C., Drake, J. F., \& Budich, W. 1977, ApJ, 216, 291

Savaglio, S., \& Fall, S. M. 2004, ApJ, 614, 293

Schady, P. 2017, R. Soc. Open Sci., 4, 170304

Schaye, J. 2001, ApJ, 562, L95
Selsing, J., Malesani, D., Goldoni, P., et al. 2019, A\&A, in press, DOI: $10.1051 / 0004-6361 / 201832835$

Sharma, S. 2017, ARA\&A, 55, 213

Sheffer, Y., Prochaska, J. X., Draine, B. T., Perley, D. A., \& Bloom, J. S. 2009, ApJ, 701, L63

Skúladóttir, A. 2010, Master Thesis, University of Copenhagen, Denmark

Sparre, M., Hartoog, O. E., Krühler, T., et al. 2014, ApJ, 785, 150

Srianand, R., Petitjean, P., Ledoux, C., Ferland, G., \& Shaw, G. 2005, MNRAS 362,549

Srianand, R., Noterdaeme, P., Ledoux, C., \& Petitjean, P. 2008, A\&A, 482, L39

Stanway, E. R., Levan, A. J., Tanvir, N. R., Wiersema, K., \& van der Laan, T. P. R. 2015, ApJ, 798, L7

Sternberg, A., Le Petit, F., Roueff, E., \& Le Bourlot J. 2014, ApJ, 790, 10

Tacconi, L. J., Genzel, R., Smail, I., et al. 2008, ApJ, 680, 246

Tanvir, N. R., Fynbo, J. P. U., de Ugarte Postigo, A., et al. 2019, MNRAS, 483, 5380

Thöne, C. C., Fynbo, J. P. U., Goldoni, P., et al. 2013, MNRAS, 428, 3590

Todini, P., \& Ferrara, A. 2001, MNRAS, 325, 726

Tumlinson, J., Prochaska, J. X., Chen, H.-W., Dessauges-Zavadsky, M., \& Bloom, J. S. 2007, ApJ, 668, 667

Ubachs, W., Salumbides, E. J., Murphy, M. T., Abgrall, H., \& Roueff, E. 2018, A\&A, 622, A127

Vernet, J., Dekker, H., D’Odorico, S., et al. 2011, A\&A, 536, A105

Vladilo, G., Gioannini, L., Matteucci, F., \& Palla, M. 2018, ApJ, 868, 127

Vreeswijk, P. M., Ledoux, C., Smette, A., et al. 2007, A\&A, 468, 83

Vreeswijk, P. M., Ledoux, C., Smette, A., et al. 2011, A\&A, 532, C3

Vreeswijk, P. M., Savaglio, S., Gal-Yam, A., et al. 2014, ApJ, 797, 24

Watson, D. 2011, A\&A, 533, A16

Wiseman, P., Perley, D. A., Schady, P., et al. 2017a, A\&A, 607, A107

Wiseman, P., Schady, P., Bolmer, J., et al. 2017b, A\&A, 599, A24

Wolfe, A. M., Gawiser, E., \& Prochaska, J. X. 2005, ARA\&A, 43, 861

Yan, L., Perley, D. A., De Cia, A., et al. 2018, ApJ, 858, 91

Zafar, T., Møller, P., Watson, D., et al. 2018a, MNRAS, 480, 108

Zafar, T., Watson, D., Møller, P., et al. 2018b, MNRAS, 1320

Zwaan, M. A., \& Prochaska, J. X. 2006, ApJ, 643, 675

1 European Southern Observatory, Alonso de Córdova 3107, Vitacura, Casilla 19001, Santiago 19, Chile

2 Max-Planck-Institut für extraterrestrische Physik, Giessenbachstraße, 85748 Garching, Germany

e-mail: jan@bolmer.de

3 School of Physics and Astronomy, University of Southampton, Southampton SO17 1BJ, UK

4 European Southern Observatory, Karl-Schwarzschild Str. 2, 85748 Garching bei München, Germany

5 The Cosmic Dawn Center, Niels Bohr Institute, Copenhagen University, Juliane Maries Vej 30, 2100 Copenhagen $\varnothing$, Denmark

6 Department of Physics, University of Bath, Claverton Down, Bath BA2 7AY, UK

7 Physics Department, University of Calabria, Arcavacata di Rende, Italy University of Calabria, 87036 Rende, Italy

8 INAF - Osservatorio Astronomico di Roma, Via Frascati 33, 00040 Monteporzio Catone, Italy

9 Astroparticule et Cosmologie, Université Paris Diderot, CNRS/IN2P3, CEA/Irfu, Observatoire de Paris, Sorbonne Paris Cité, 10 Rue Alice Domon et Léonie Duquet, 75205 Paris Cedex 13, France

10 Department of Physics and Astronomy, Clemson University, Clemson, SC 29634-0978, USA

11 Centre for Astrophysics and Cosmology, Science Institute, University of Iceland, Dunhagi 5, 107 Reykjavík, Iceland

12 Anton Pannekoek Institute for Astronomy, University of Amsterdam, Science Park 904, 1098 XH Amsterdam, The Netherlands

13 Department of Physics and Astronomy and Leicester Institute of Space and Earth Observation, University of Leicester, University Road, Leicester LE1 7RH, UK

14 Department of Astrophysics/IMAPP, Radboud University, PO Box 9010, 6500 GL Nijmegen, The Netherlands

15 Australian Astronomical Observatory, PO Box 915, North Ryde, NSW 1670, Australia 
J. Bolmer et al.: Evidence for diffuse molecular gas and dust in the hearts of gamma-ray burst host galaxies

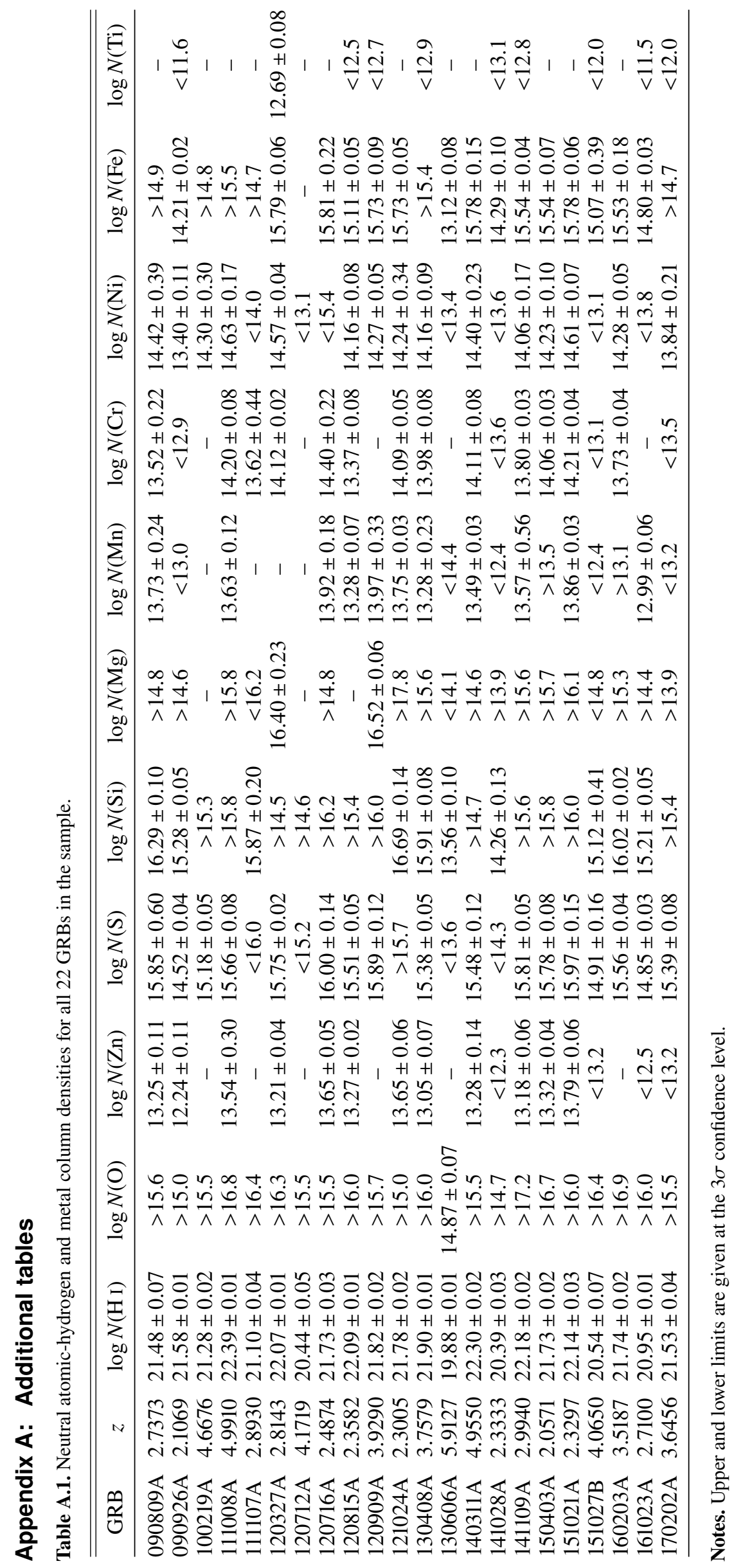




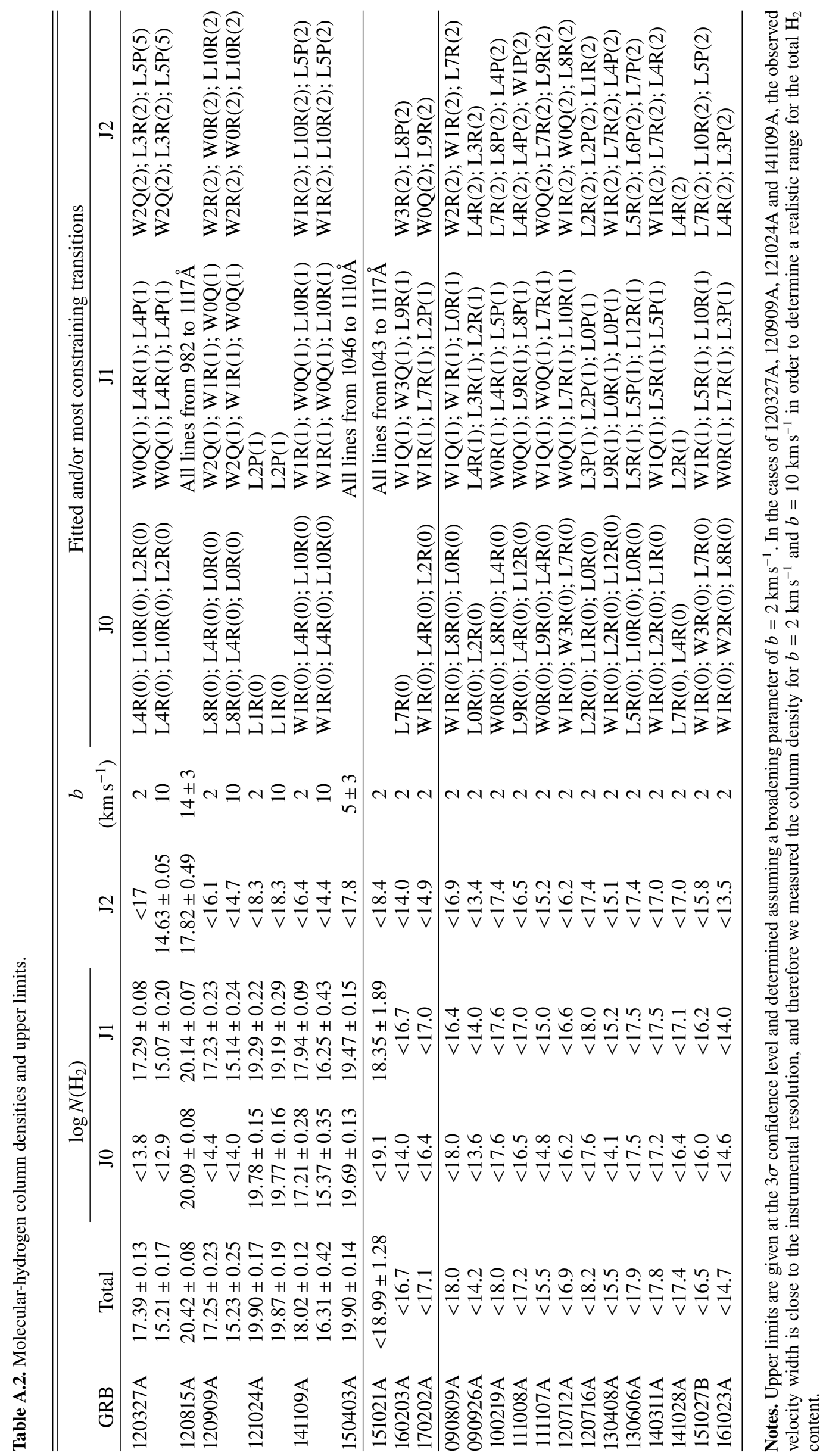




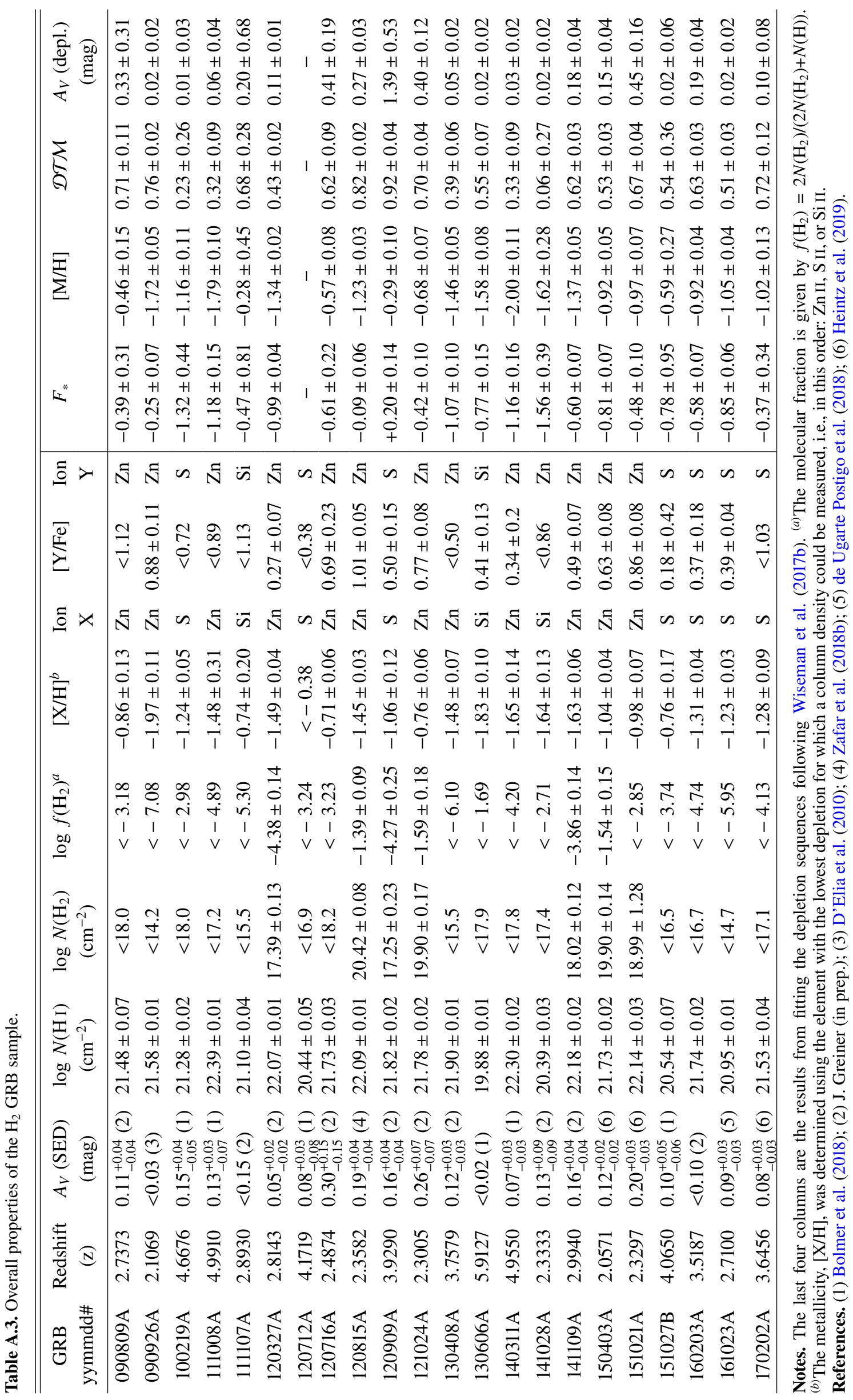


Appendix B: Additional plots

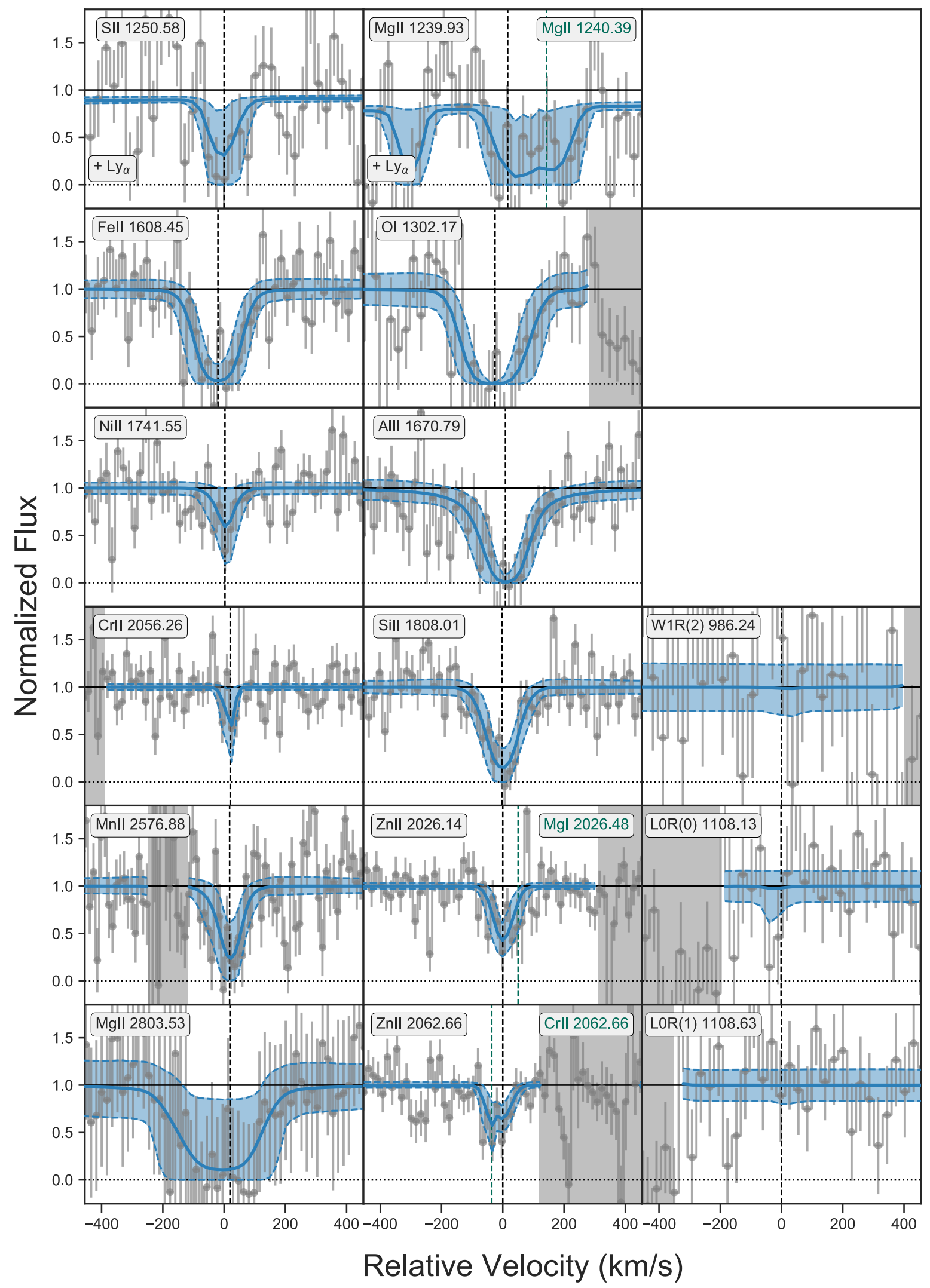

Fig. B.1. Results from fitting absorption lines in the X-shooter spectrum of GRB 090809A. As shown to the right, we do not find any evidence for absorption from molecular hydrogen. This X-shooter spectrum was previously also analysed by Skúladóttir (2010). 
J. Bolmer et al.: Evidence for diffuse molecular gas and dust in the hearts of gamma-ray burst host galaxies

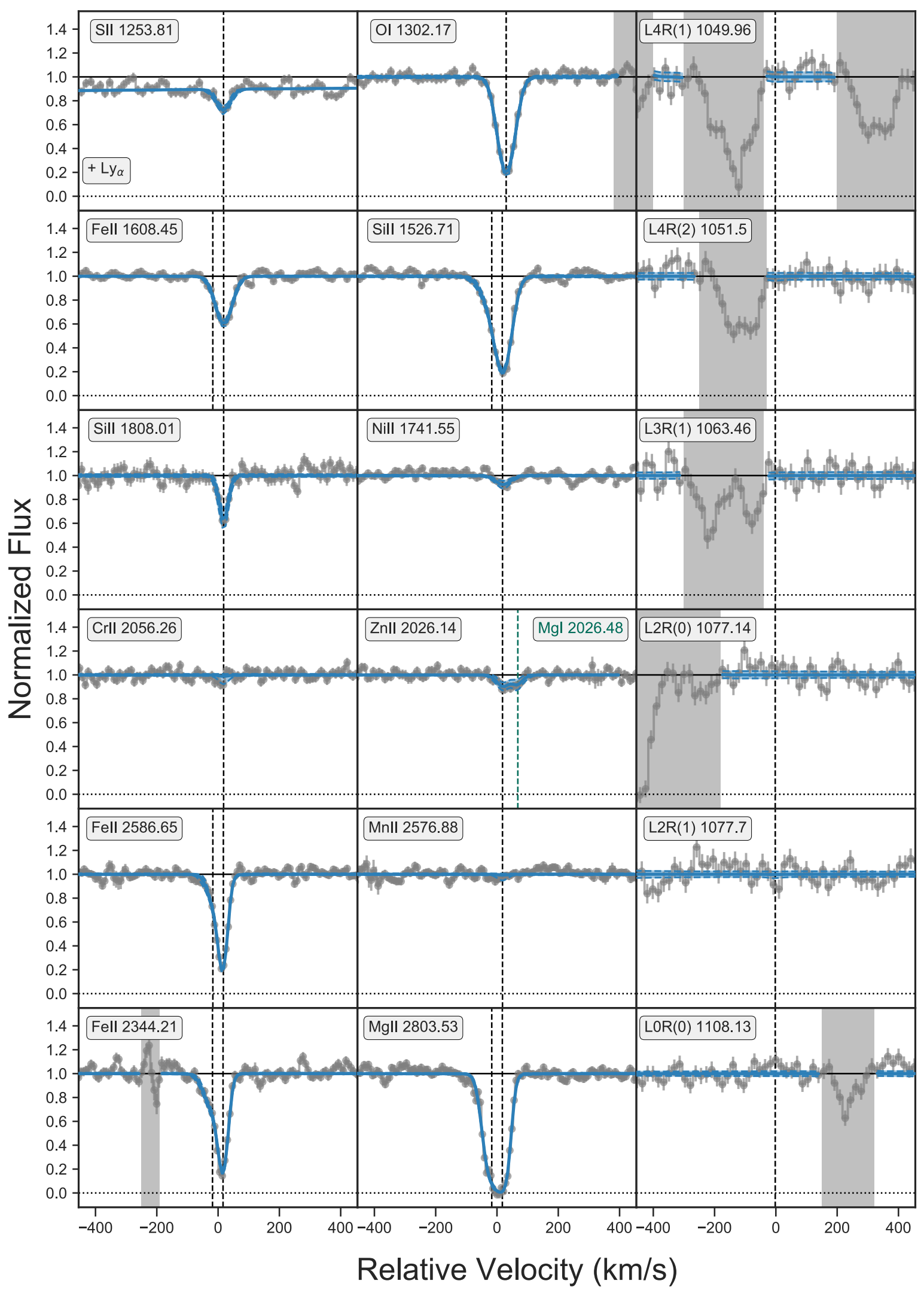

Fig. B.2. Results from fitting absorption lines in the X-shooter spectrum of GRB 090926A. As shown to the right, we do not find any evidence for absorption from molecular hydrogen. This X-shooter spectrum was previously also analysed by D'Elia et al. (2010). 


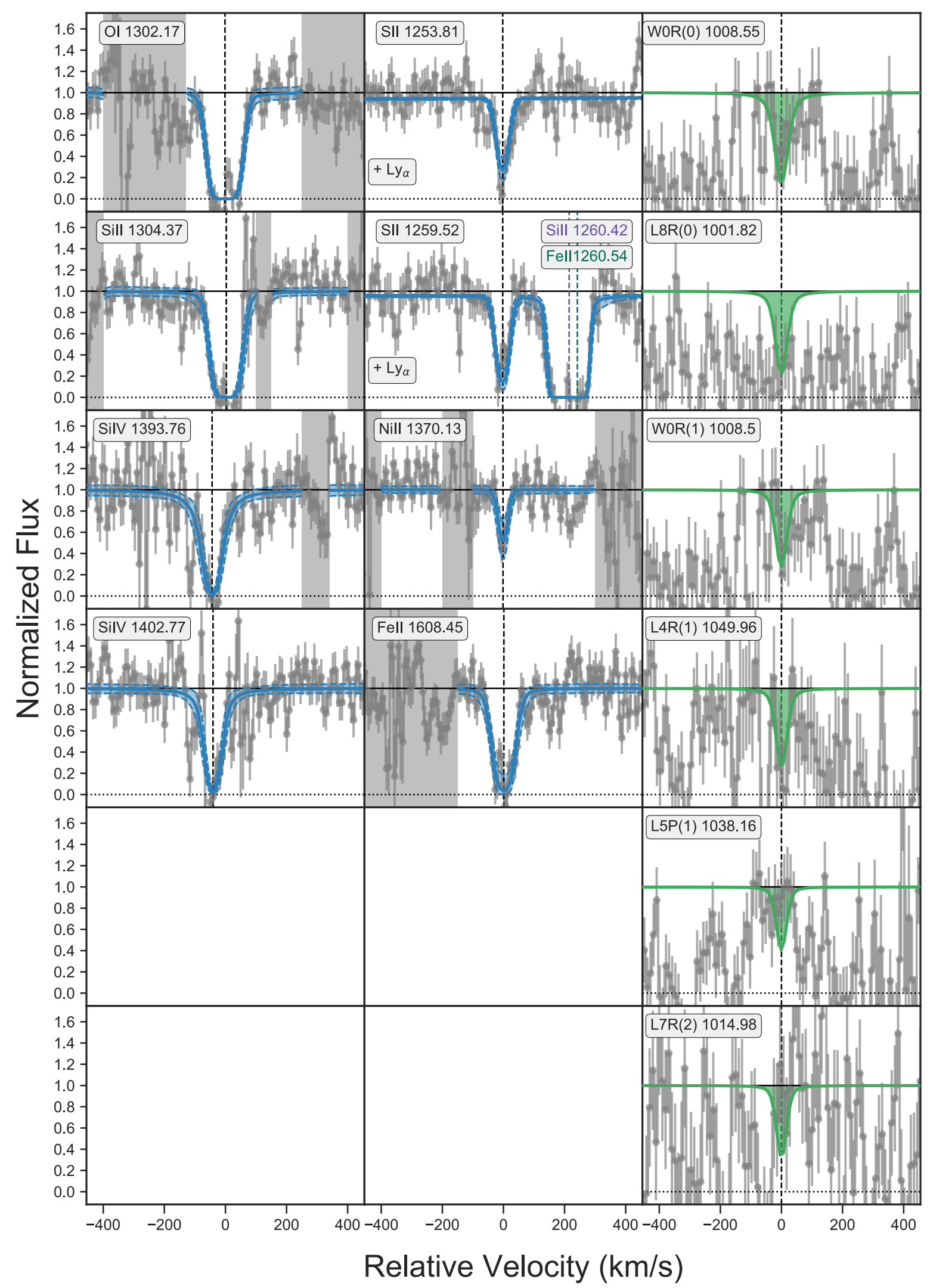

Fig. B.3. Results from fitting absorption lines in the X-shooter spectrum of GRB 100219A. As shown to the right, we do not find compelling evidence for absorption from molecular hydrogen, however, due to the strong Lyman- $\alpha$ forest, only down to relatively loose upper limits (see Table A.2). These upper limits are over-plotted in green for the 6 most constraining lines. This X-shooter spectrum was previously also analysed by Thöne et al. (2013). 
J. Bolmer et al.: Evidence for diffuse molecular gas and dust in the hearts of gamma-ray burst host galaxies

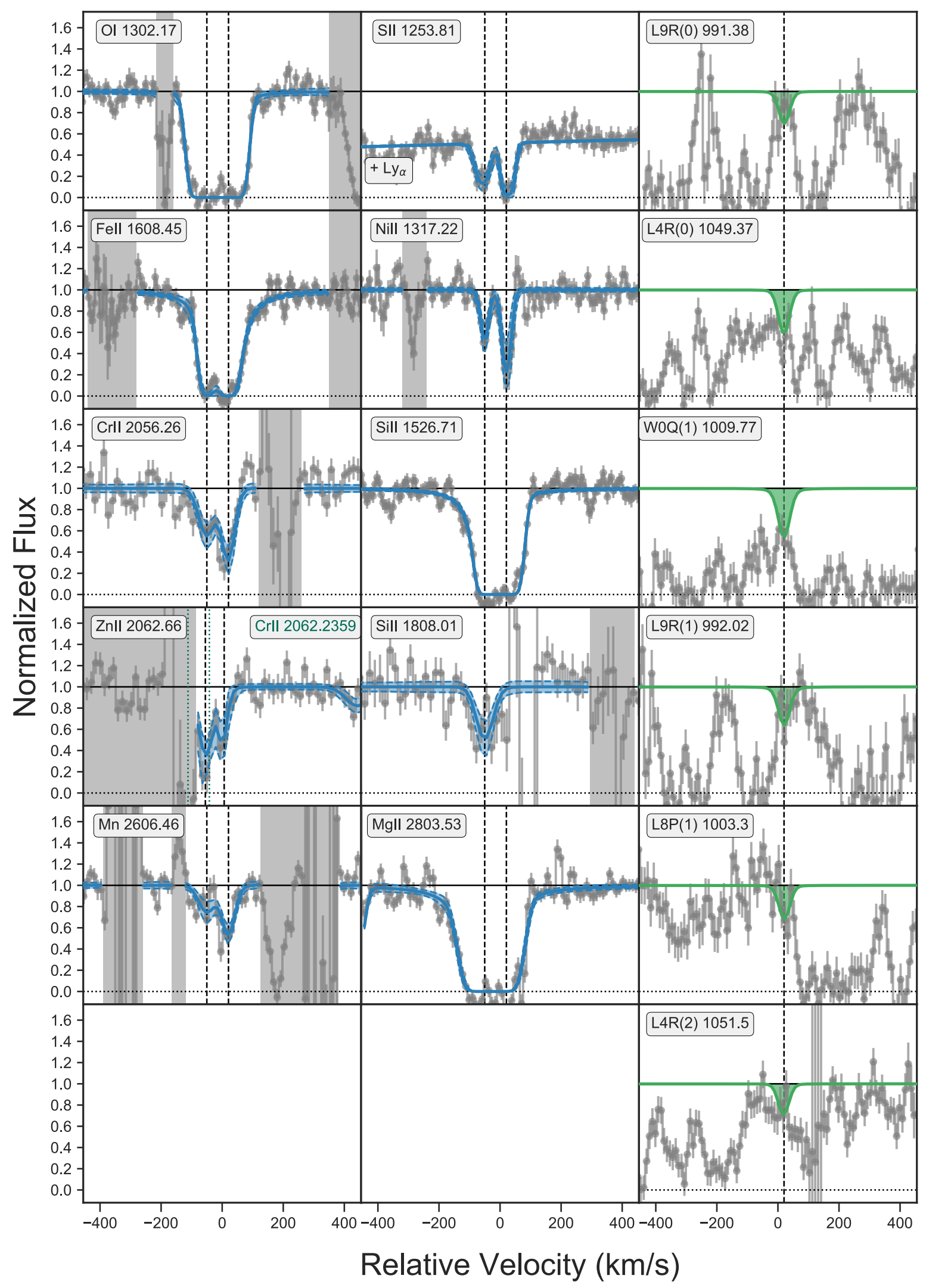

Fig. B.4. Results from fitting absorption lines in the X-shooter spectrum of GRB 111008A. As shown to the right, we do not find compelling evidence for absorption from molecular hydrogen, however, due to the strong Lyman- $\alpha$ forest, only down to relatively loose upper limits (see Table A.2). These upper limits are over-plotted in green for the 6 most constraining lines. This X-shooter spectrum was previously also analysed by Sparre et al. (2014). 


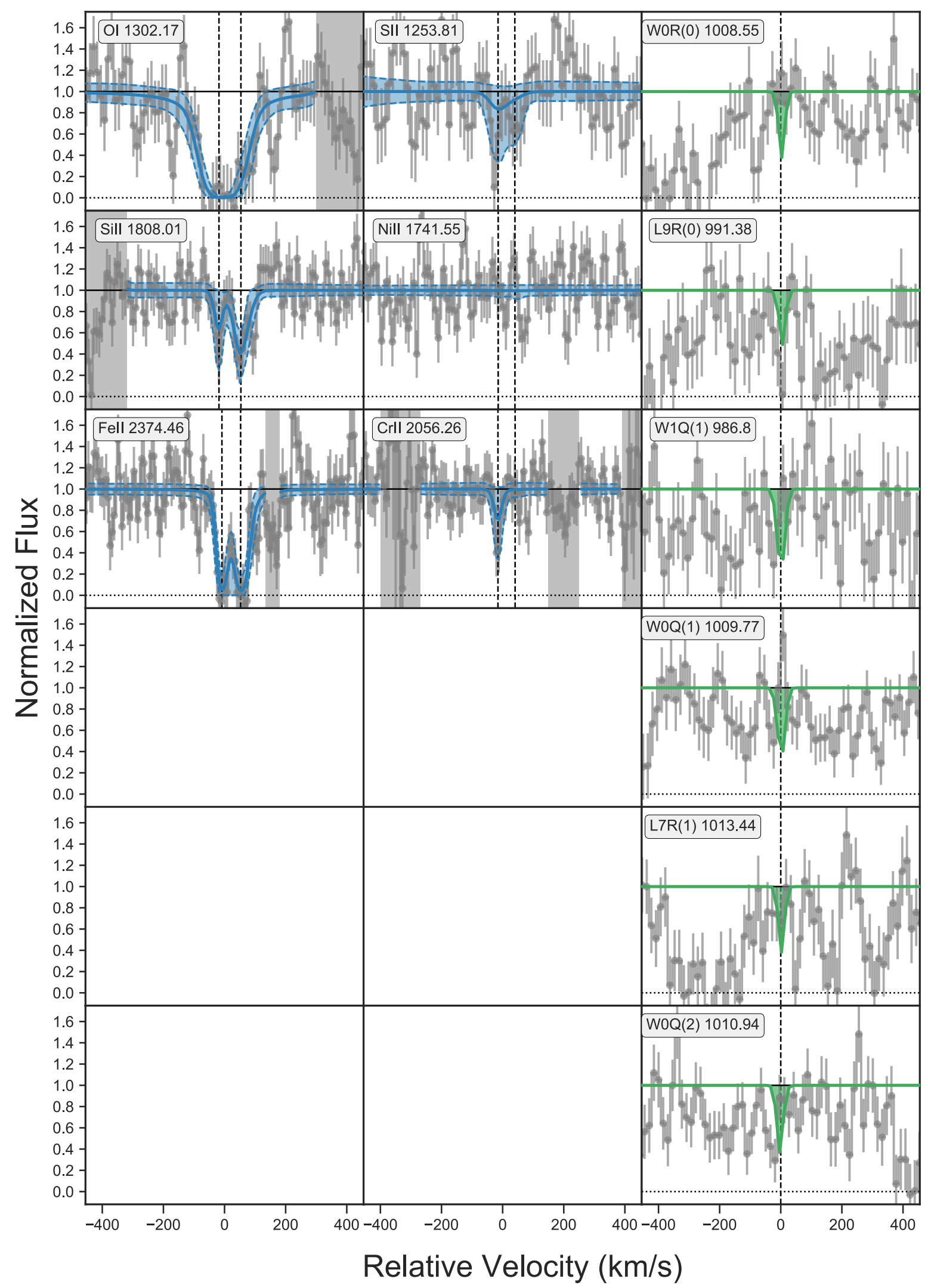

Fig. B.5. Results from fitting absorption lines in the X-shooter spectrum of GRB 111107A. As shown to the right, we do not find evidence for absorption from molecular hydrogen down to the upper limits given in Table A.2. These upper limits are over-plotted in green for the 6 most constraining lines. 
J. Bolmer et al.: Evidence for diffuse molecular gas and dust in the hearts of gamma-ray burst host galaxies

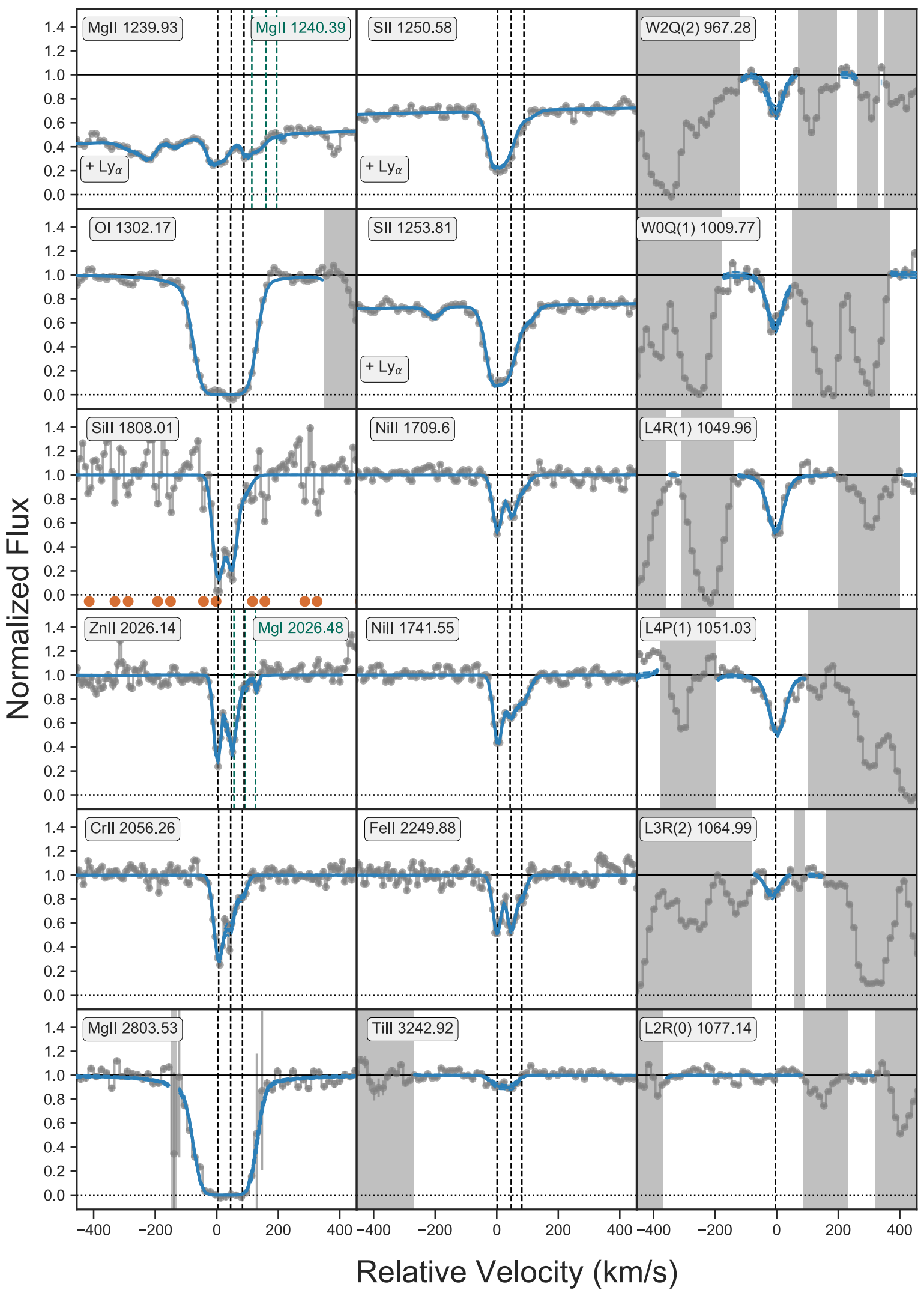

Fig. B.6. Results from fitting absorption lines in the X-shooter spectrum of GRB 120327A. The position of telluric lines, which were corrected, are marked by red dots. As shown to the right, we find absorption lines from molecular hydrogen consistent with the strongest metal component. This X-shooter spectrum was previously also analysed by D’Elia et al. (2014). 


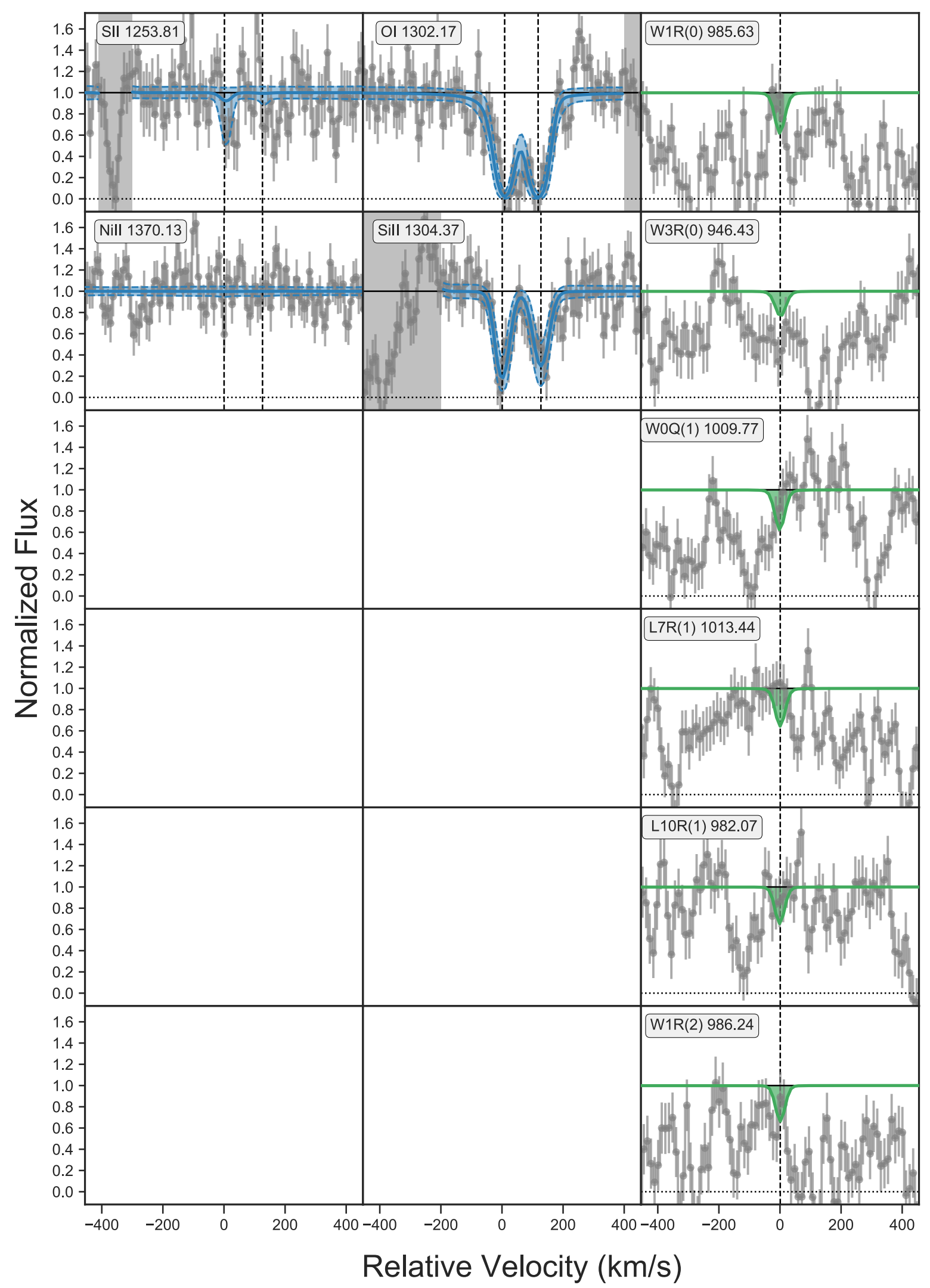

Fig. B.7. Results from fitting absorption lines in the X-shooter spectrum of GRB 120712A. As shown to the right, we do not find convincing evidence for absorption from molecular hydrogen down to the upper limits given in Table A.2. These upper limits are over-plotted in green for the 6 most constraining lines. 
J. Bolmer et al.: Evidence for diffuse molecular gas and dust in the hearts of gamma-ray burst host galaxies

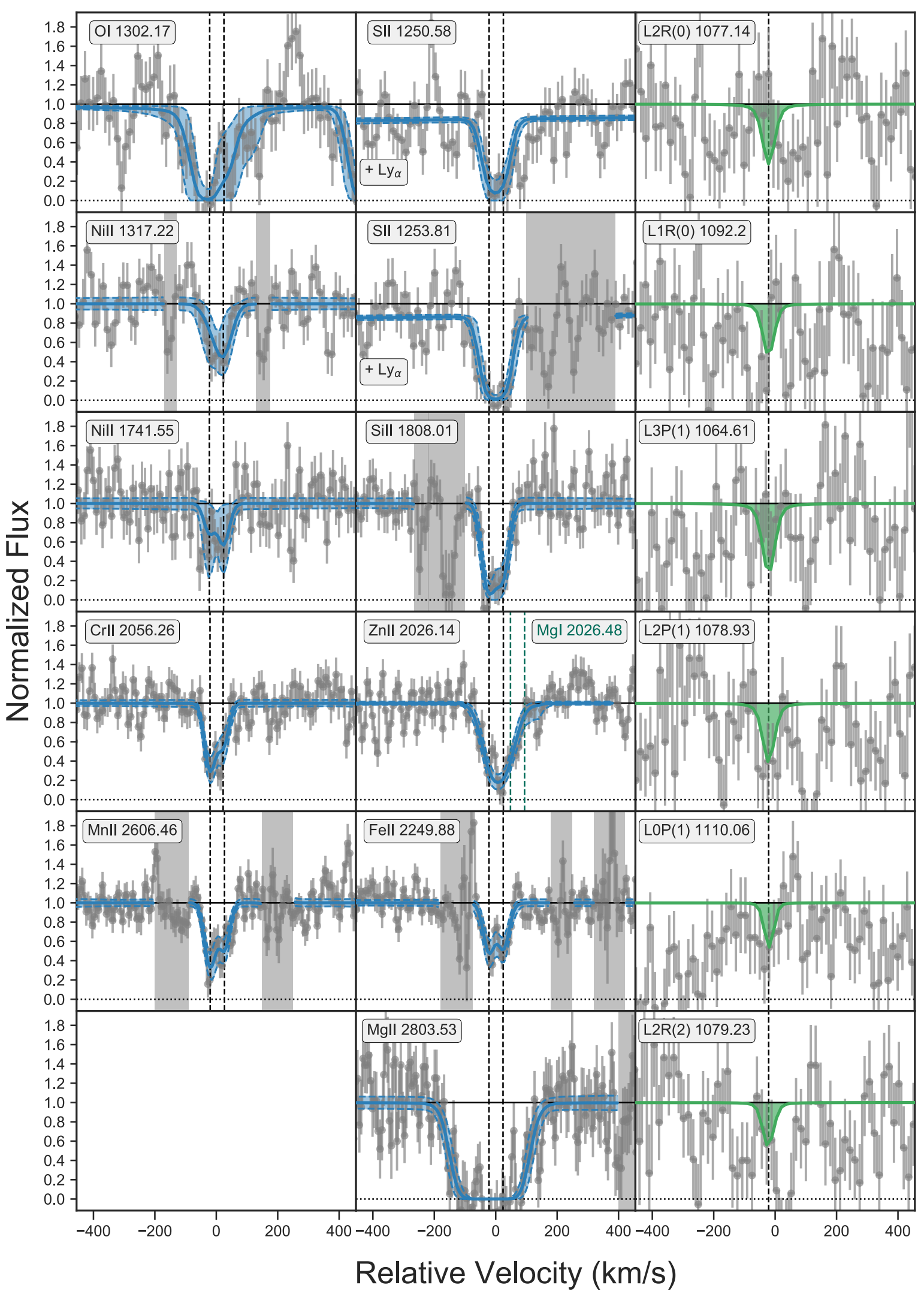

Fig. B.8. Results from fitting absorption lines in the X-shooter spectrum of GRB 120716A. As shown to the right, we do not find compelling evidence for absorption from molecular hydrogen, however, due to the poor $\mathrm{S} / \mathrm{N}$, only down to relatively loose upper limits (see Table A.2). These upper limits are over-plotted in green for the 6 most constraining lines. This X-shooter spectrum was previously also analysed by Wiseman et al. (2017b). 


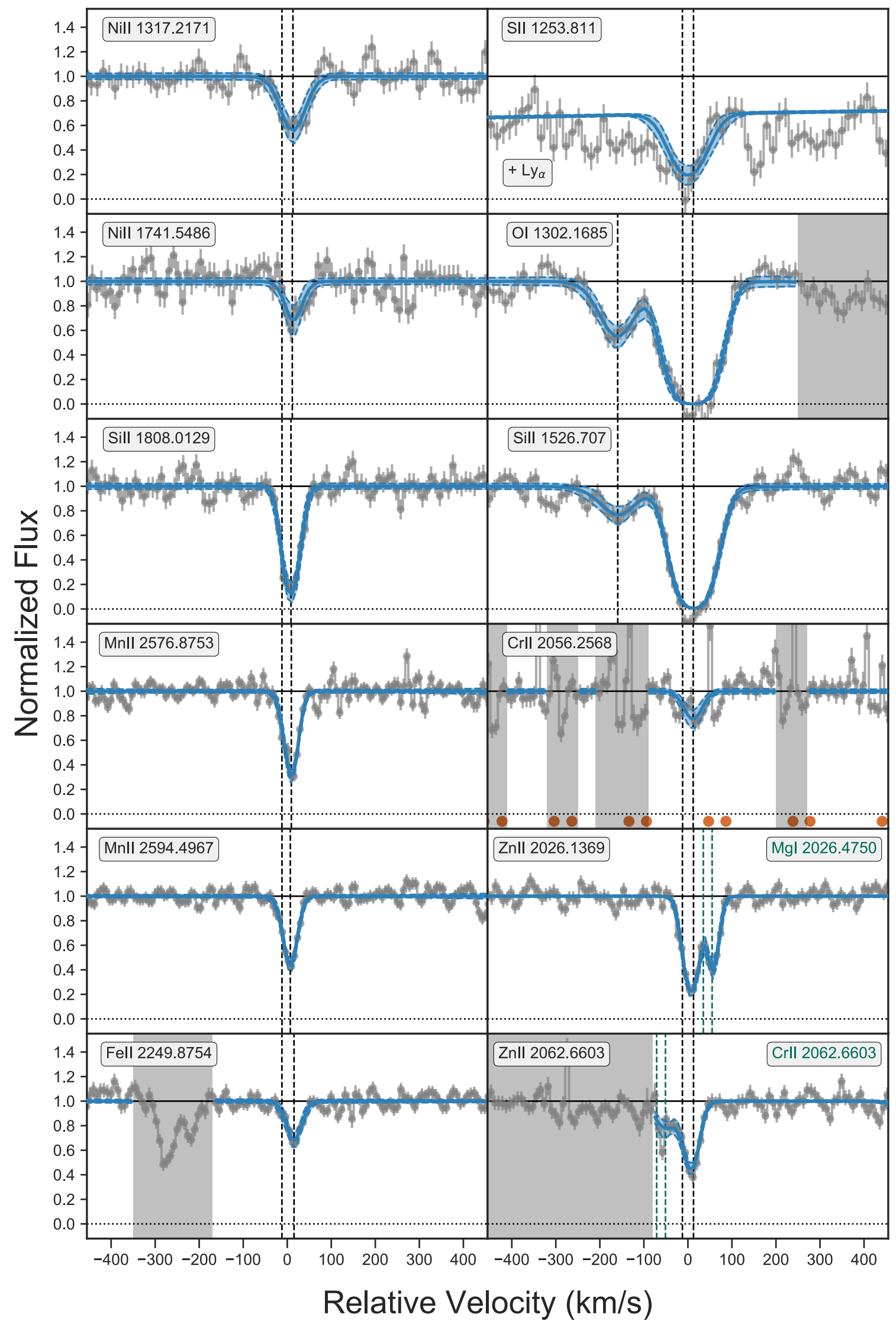

Fig. B.9. Results from fitting absorption lines in the X-shooter spectrum of GRB 120815A. The result of fitting the $\mathrm{H}_{2}$ lines is shown in Fig. 9. This $\mathrm{X}$-shooter spectrum was previously also analysed by Krühler et al. (2013). 
J. Bolmer et al.: Evidence for diffuse molecular gas and dust in the hearts of gamma-ray burst host galaxies

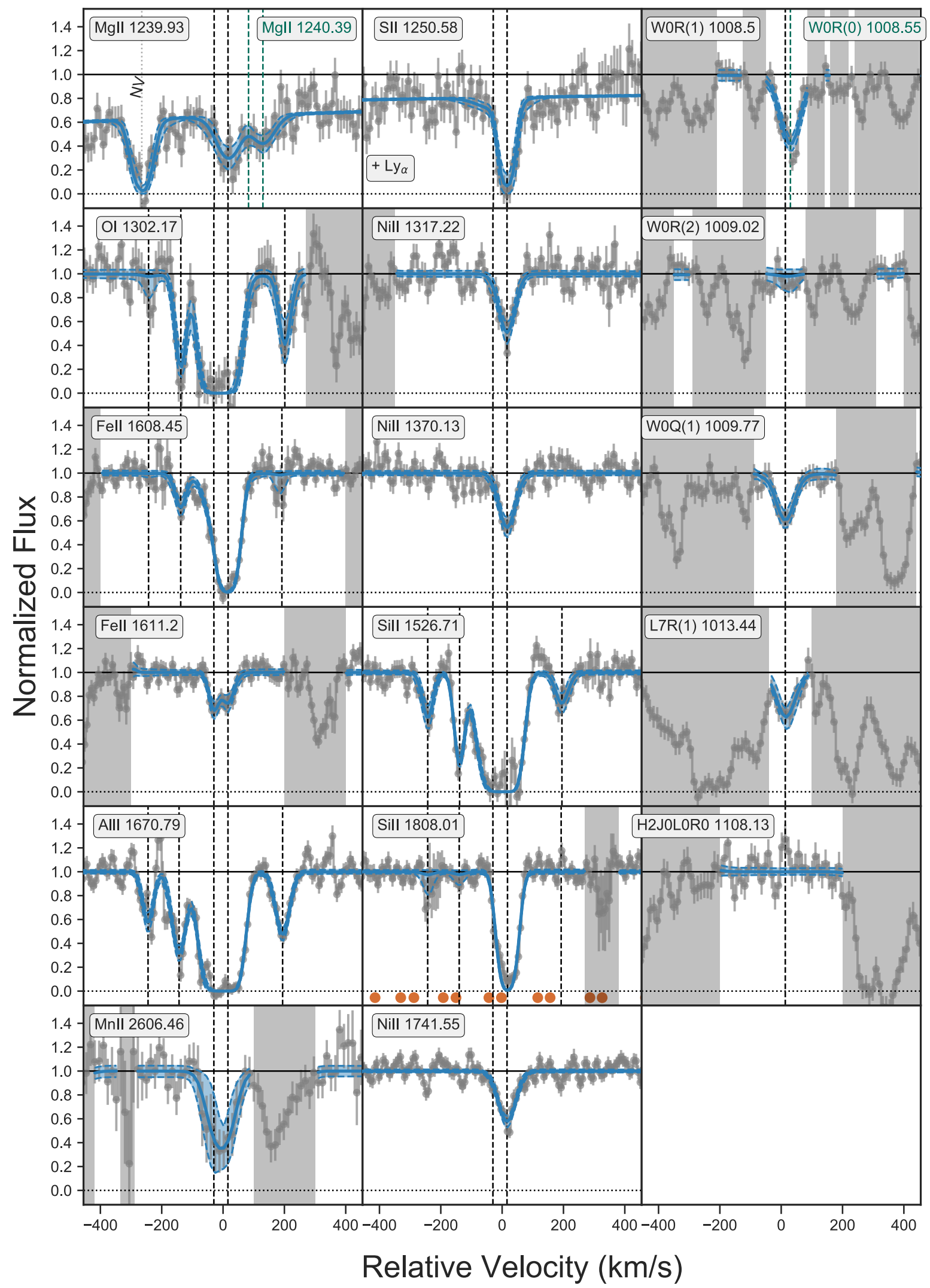

Fig. B.10. Results from fitting absorption lines in the X-shooter spectrum of GRB 120909A. As shown to the right, we find evidence for absorption from molecular hydrogen consistent with the strongest metal component. This X-shooter spectrum was previously also analysed by Wiseman et al. (2017b). 


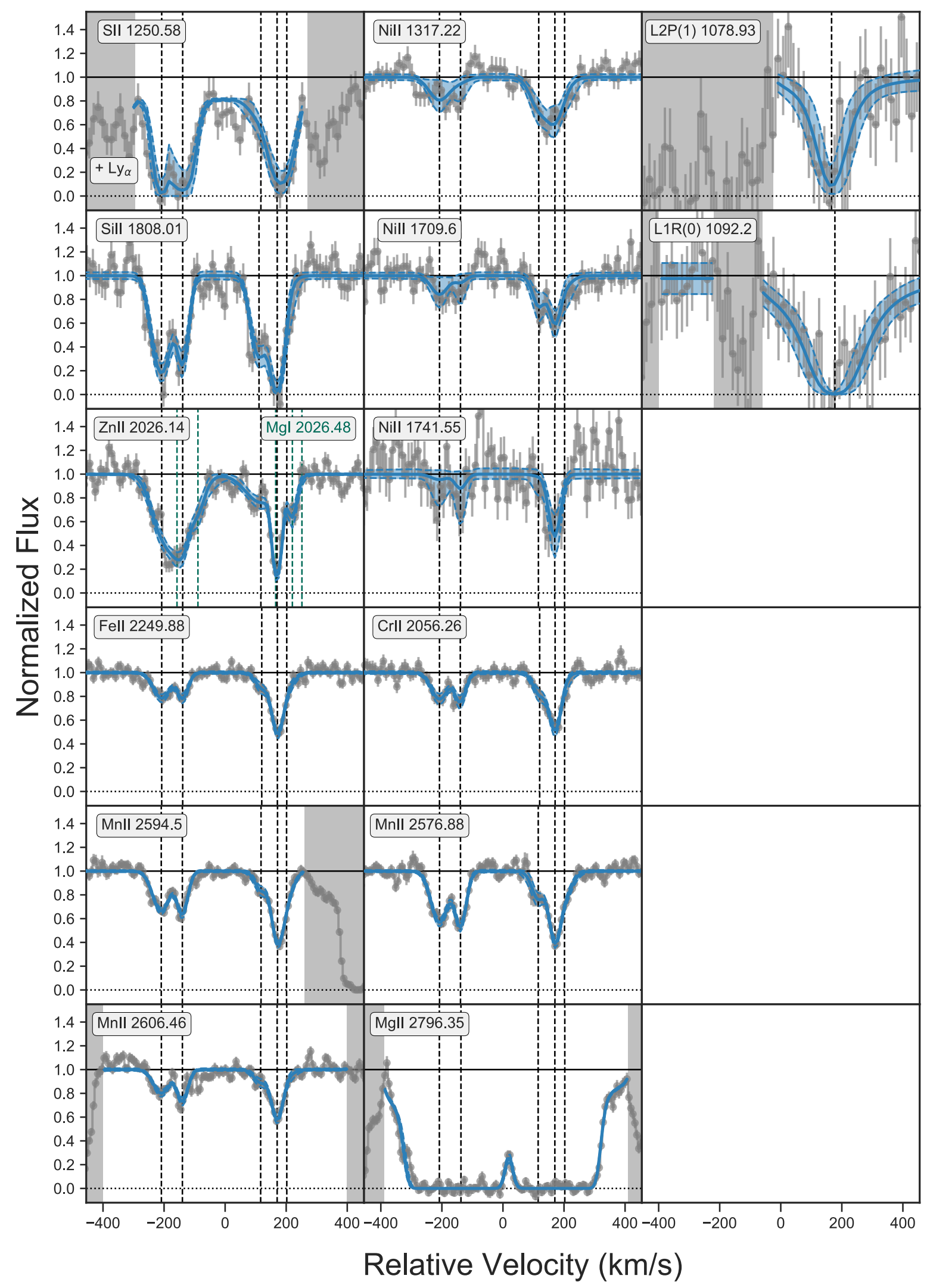

Fig. B.11. Results from fitting absorption lines in the X-shooter spectrum of GRB 121024A. As shown to the right, we find evidence for absorption from molecular hydrogen consistent with the three red components. This X-shooter spectrum was previously also analysed by Friis et al. (2015). 
J. Bolmer et al.: Evidence for diffuse molecular gas and dust in the hearts of gamma-ray burst host galaxies

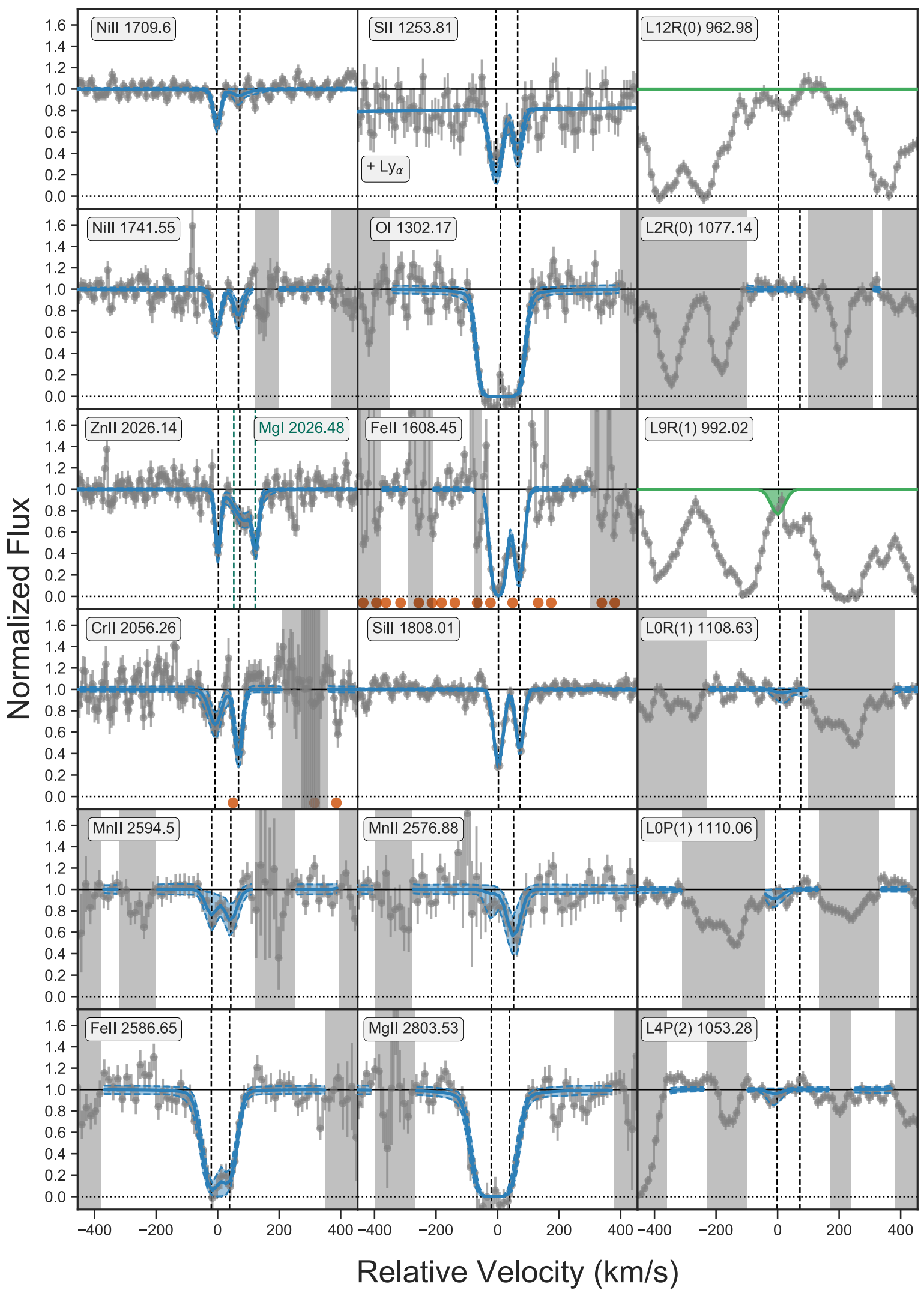

Fig. B.12. Results from fitting absorption lines in the X-shooter spectrum of GRB 130408A. As shown to the right, we do not find evidence for absorption from molecular hydrogen down to the upper limits given in Table A.2. These upper limits were derived from the fits shown in blue and are additionally over-plotted for two additional lines in green. This X-shooter spectrum was previously also analysed by Wiseman et al. (2017b). 


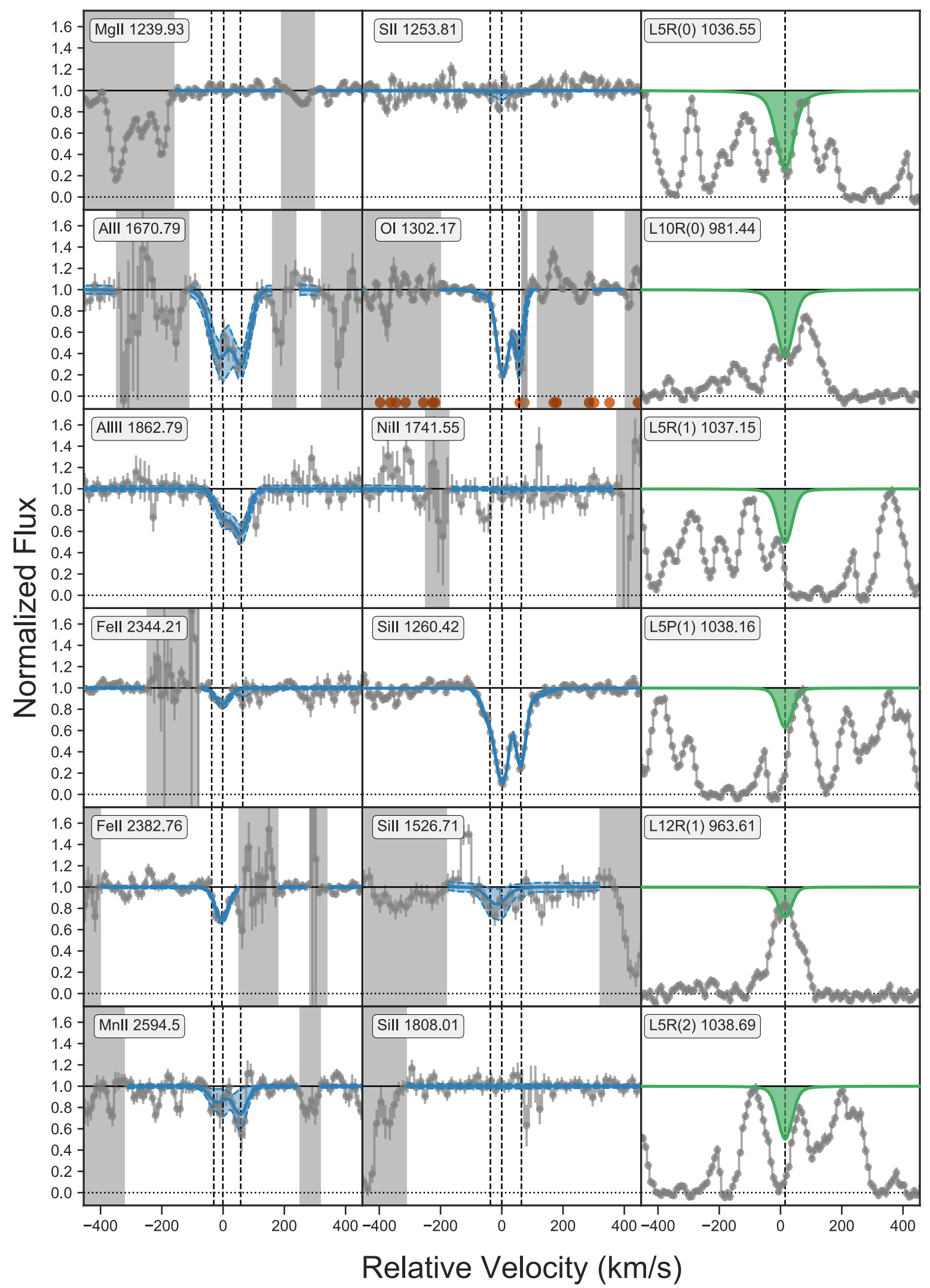

Fig. B.13. Results from fitting absorption lines in the X-shooter spectrum of GRB 130606A. As shown to the right, we do not find compelling evidence for absorption from molecular hydrogen, however, due to the strong Lyman- $\alpha$ forest, only down to relatively loose upper limits (see Table A.2). These upper limits are over-plotted in green for the 6 most constraining lines. This X-shooter spectrum was previously also analysed by Hartoog et al. (2015). 
J. Bolmer et al.: Evidence for diffuse molecular gas and dust in the hearts of gamma-ray burst host galaxies

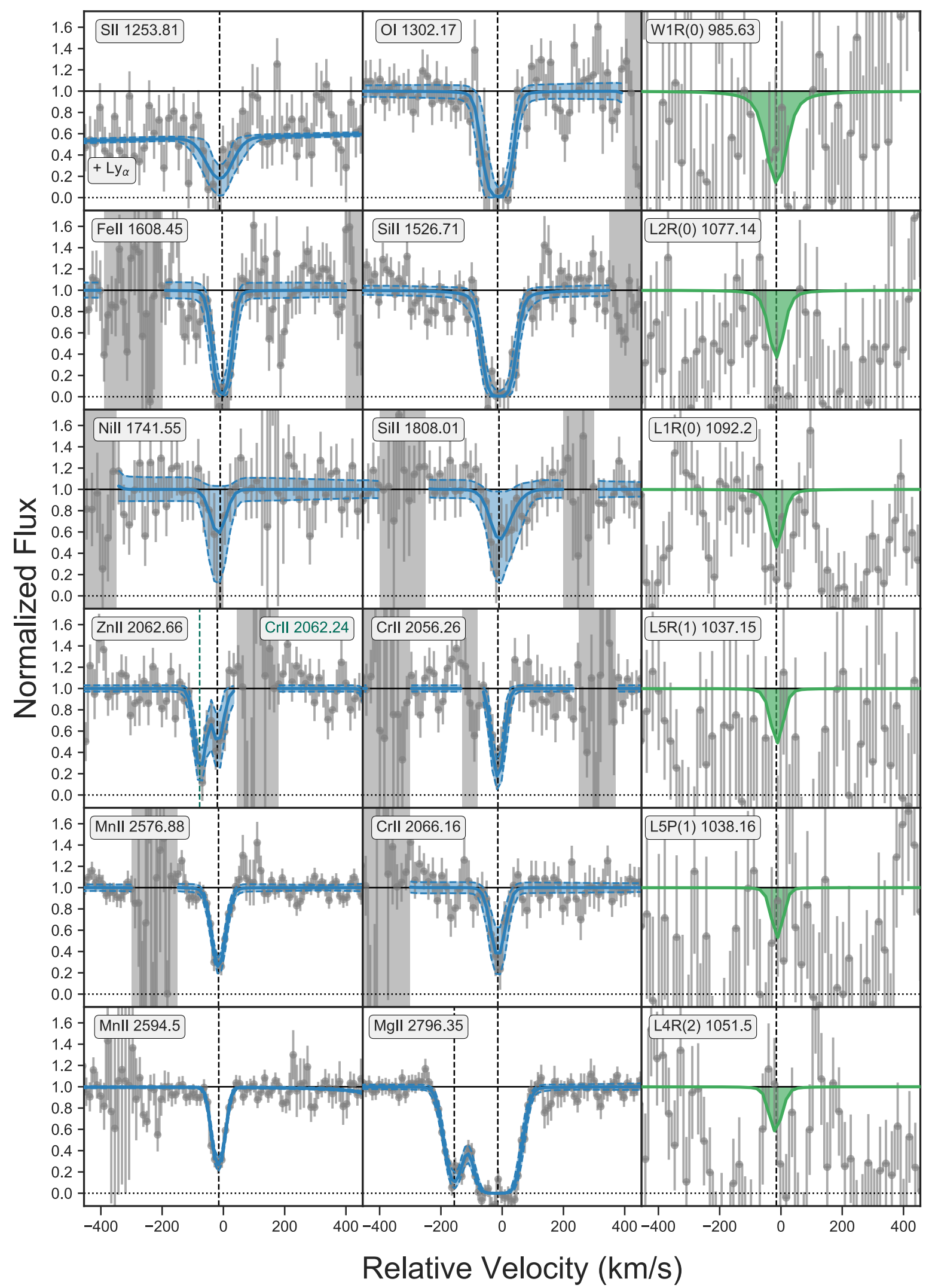

Fig. B.14. Results from fitting absorption lines in the X-shooter spectrum of GRB 140311A. As shown to the right, we do not find compelling evidence for absorption from molecular hydrogen, however, due to the poor S/N, only down to relatively loose upper limits (see Table A.2). These upper limits are over-plotted in green for the 6 most constraining lines. 


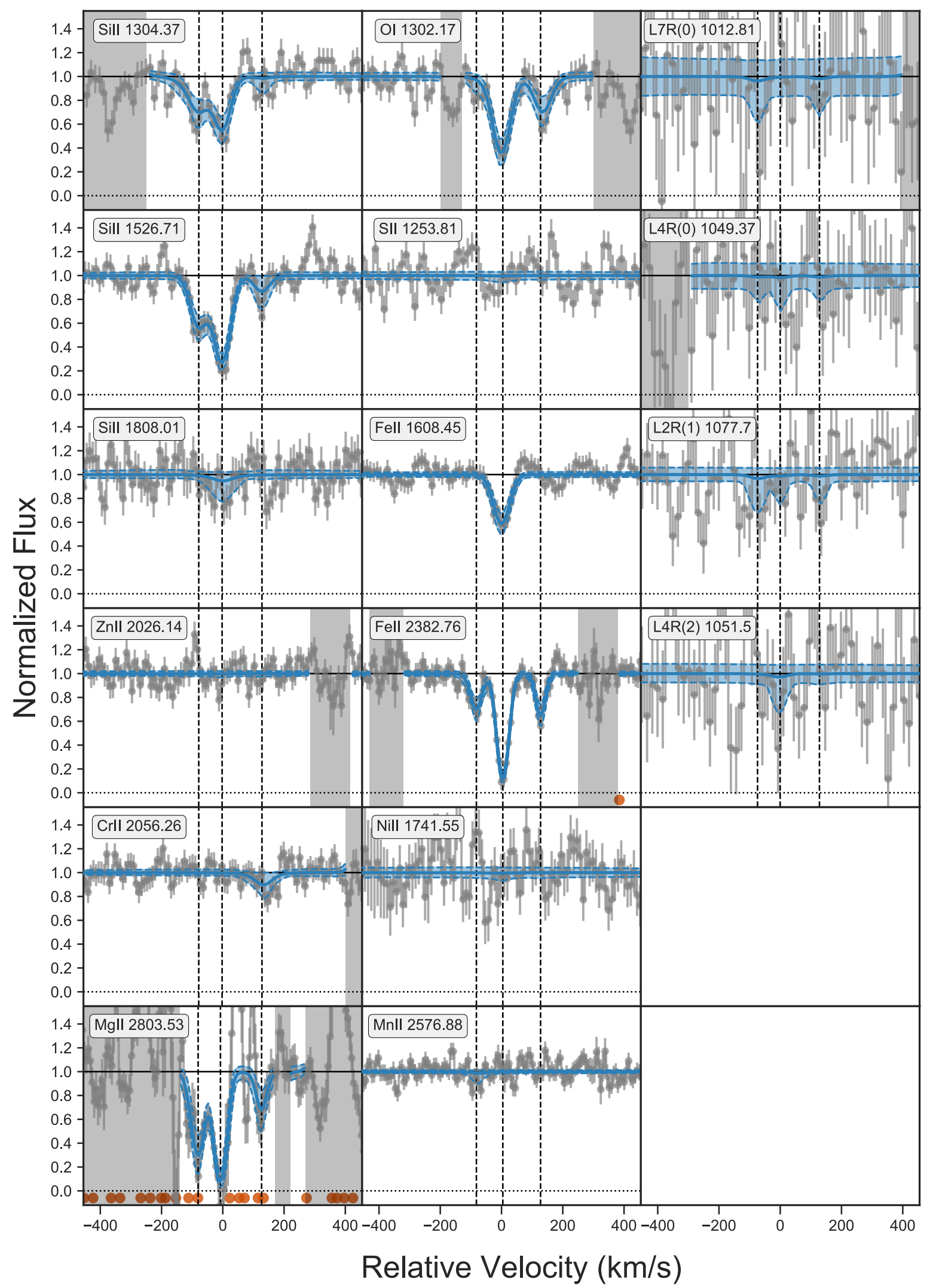

Fig. B.15. Results from fitting absorption lines in the X-shooter spectrum of GRB 141028A. The position of telluric lines, which were corrected, are marked by red dots. As shown to the right, we do not find evidence for absorption from molecular hydrogen, however, due to the poor S/N, only down to relatively loose upper limits (see Table A.2). This X-shooter spectrum was previously also analysed by Wiseman et al. (2017b). 
J. Bolmer et al.: Evidence for diffuse molecular gas and dust in the hearts of gamma-ray burst host galaxies

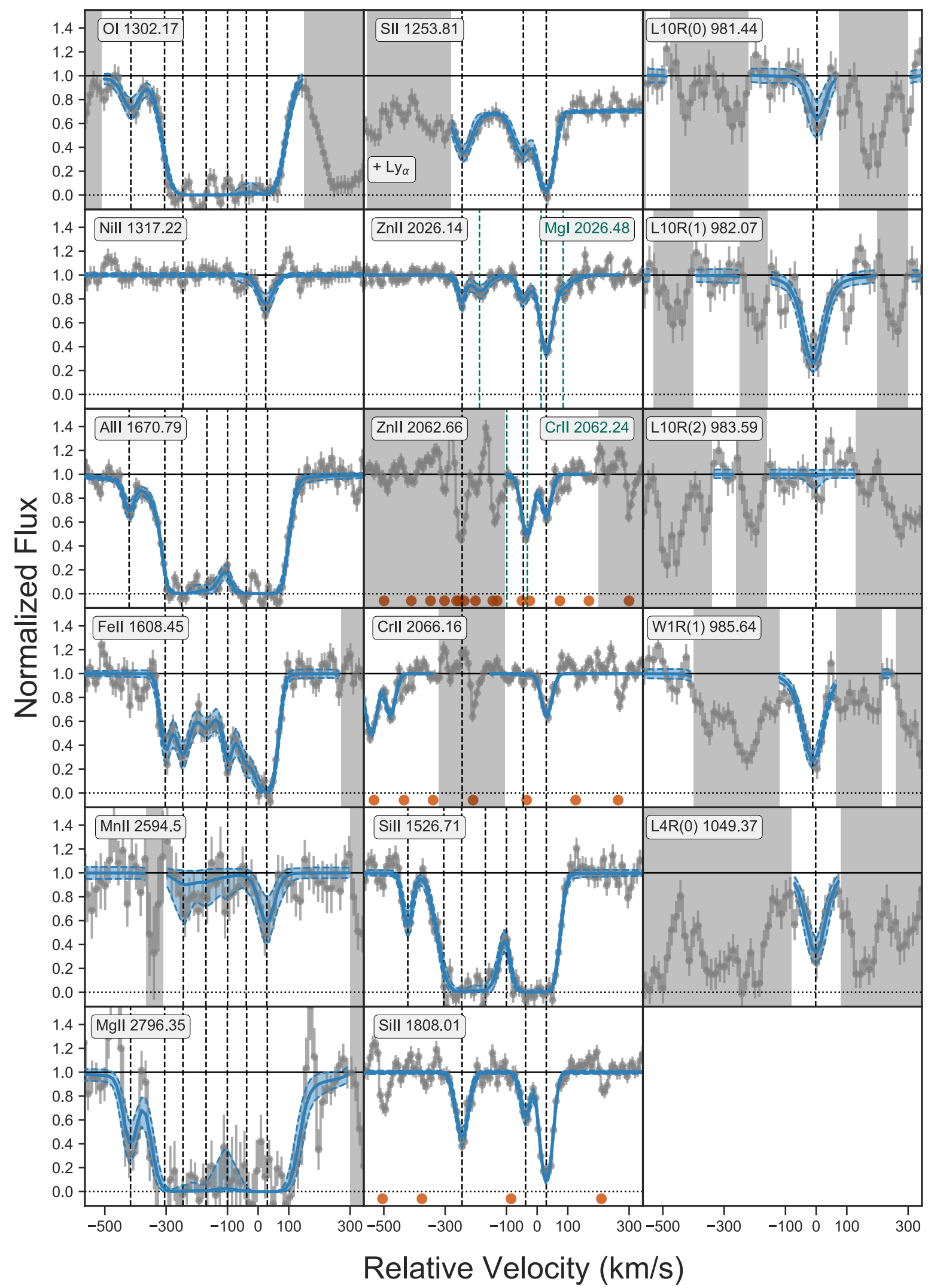

Fig. B.16. Results from fitting absorption lines in the X-shooter spectrum of GRB 141109A. The position of telluric lines, which were corrected, are marked by red dots. As shown to the right, we find absorption lines from molecular hydrogen consistent with the two red components. 


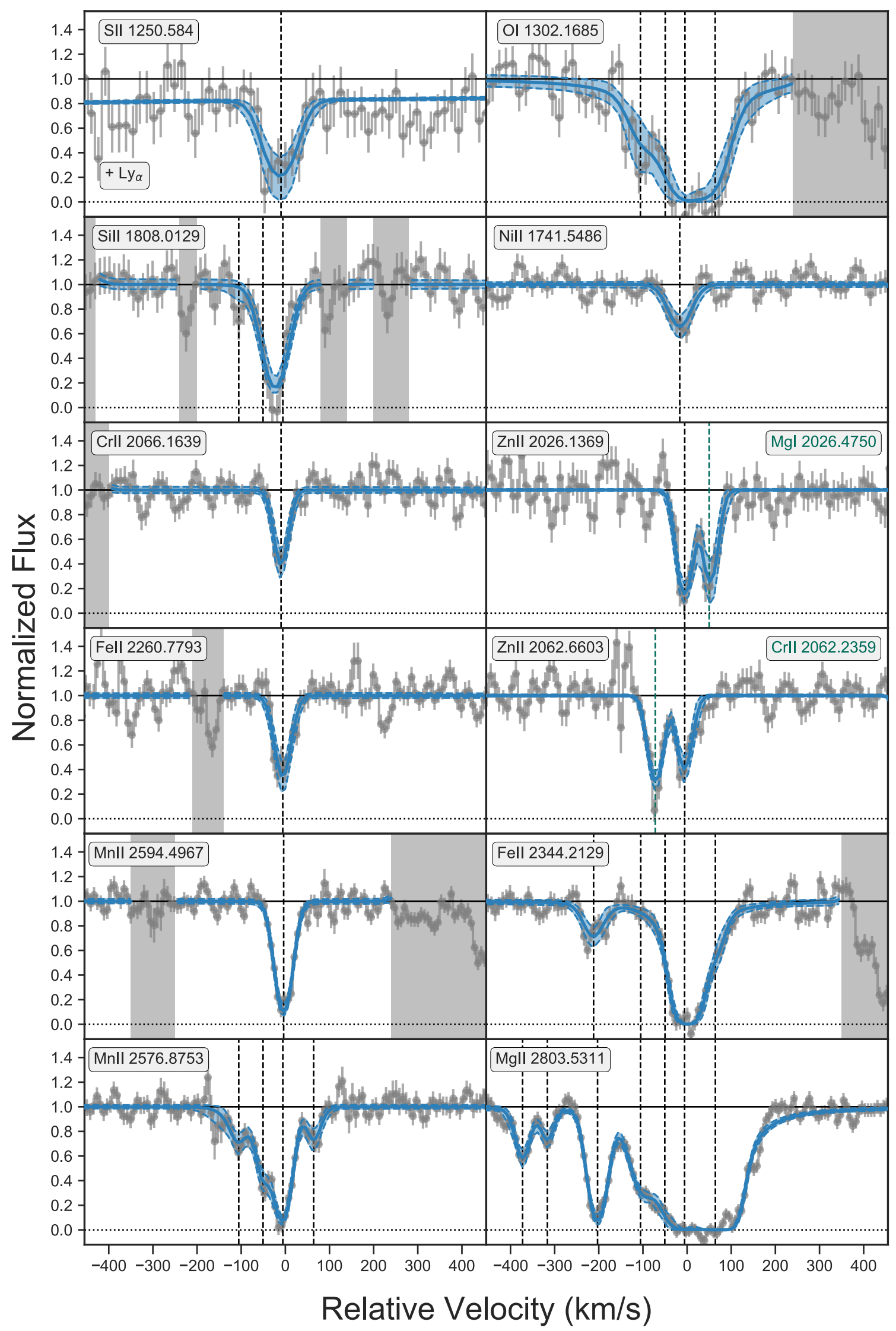

Fig. B.17. Results from fitting absorption lines in the X-shooter spectrum of GRB 150403A. The result of fitting the $\mathrm{H}_{2}$ lines is shown in Fig. 10. 
J. Bolmer et al.: Evidence for diffuse molecular gas and dust in the hearts of gamma-ray burst host galaxies

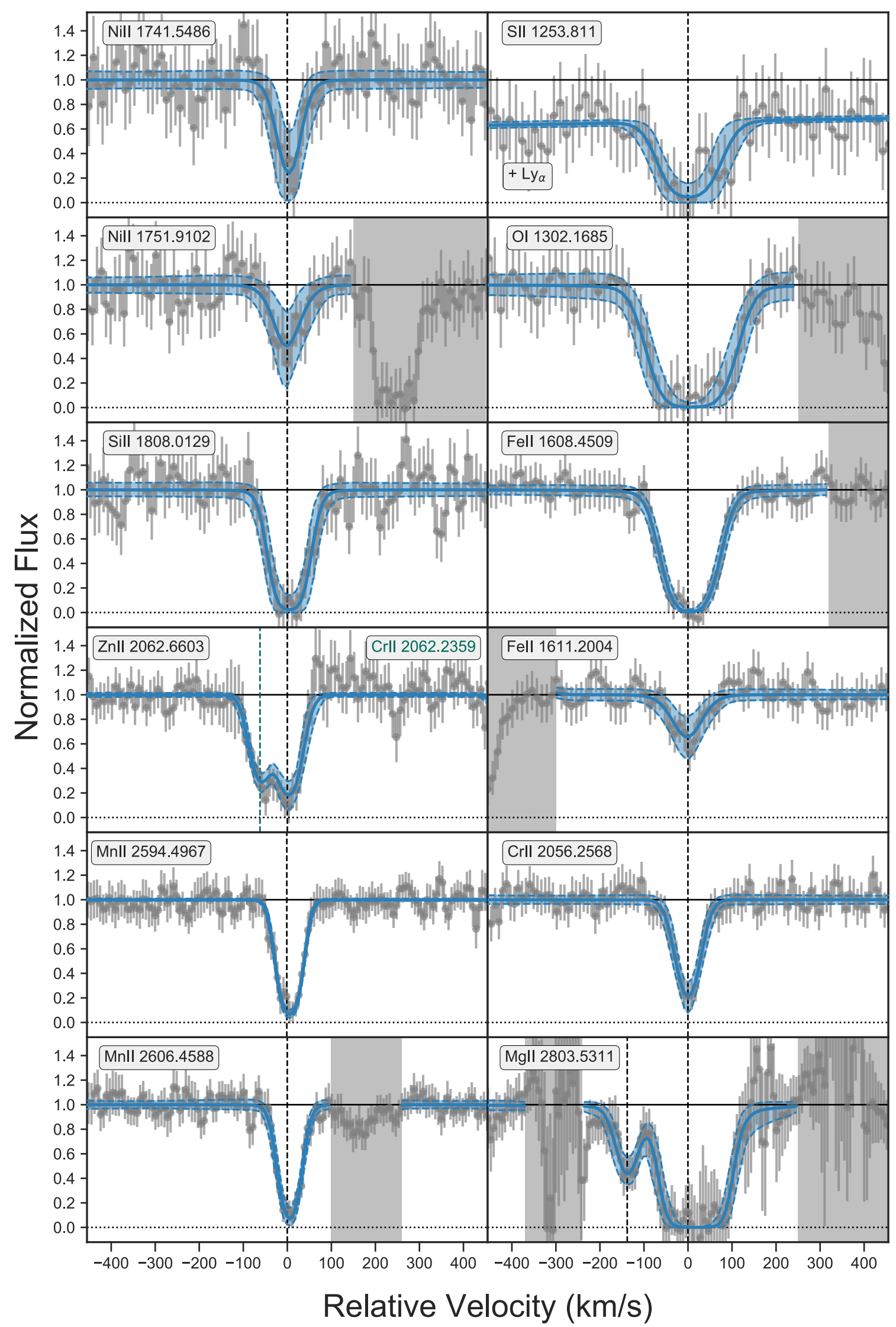

Fig. B.18. Results from fitting absorption lines in the X-shooter spectrum of GRB 151021A. The result of fitting the $\mathrm{H}_{2}$ lines is shown in Fig. 11 . 


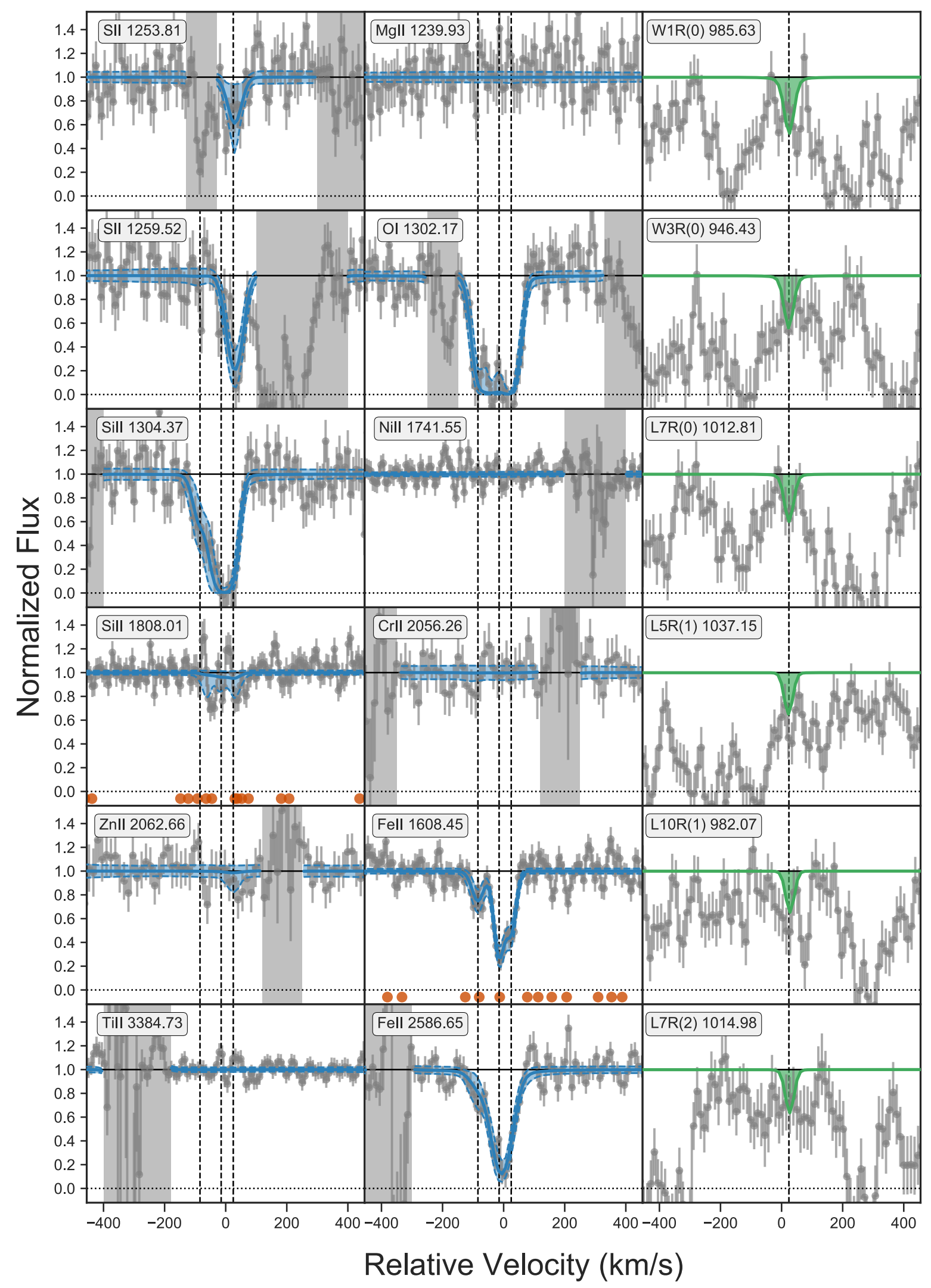

Fig. B.19. Results from fitting absorption lines in the X-shooter spectrum of GRB 151027B. The position of telluric lines, which were corrected, are marked by red dots. We do not find compelling evidence for absorption from molecular hydrogen down to the limits given in Table A.2. To the right, in green, we plot synthetic spectra for these limits 
J. Bolmer et al.: Evidence for diffuse molecular gas and dust in the hearts of gamma-ray burst host galaxies

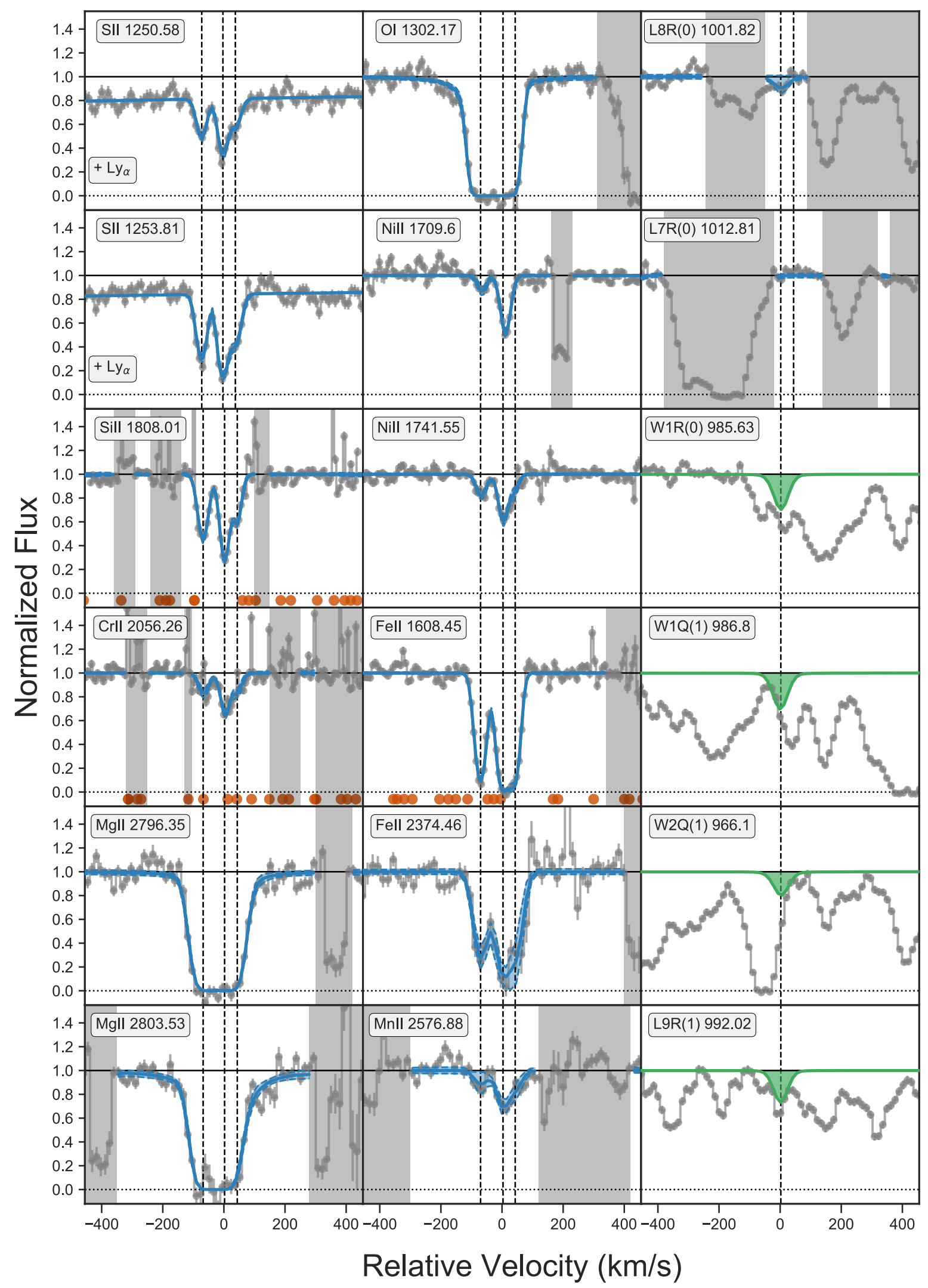

Fig. B.20. Results from fitting absorption lines in the X-shooter spectrum of GRB 160203A. The position of telluric lines, which were corrected, are marked by red dots. As shown in the 6 subplots to the right, the spectrum is consistent with absorption from molecular hydrogen, but only to the degree of claiming a possible detection. In green, we plot synthetic spectra for the upper limits given in Table A.2. 


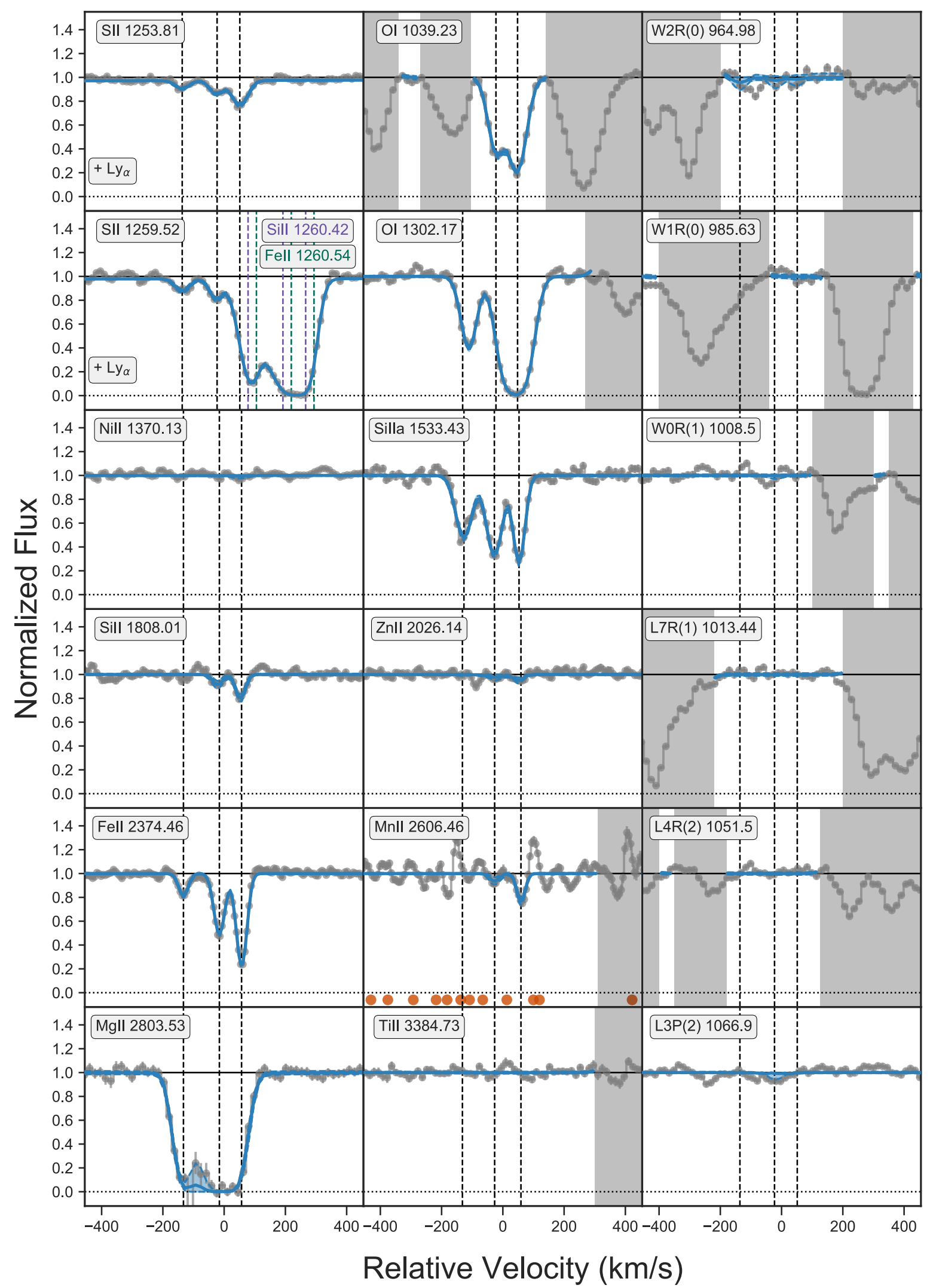

Fig. B.21. Results from fitting absorption lines in the X-shooter spectrum of GRB 161023A. The position of telluric lines, which were corrected, are marked by red dots. As shown to the right, we do not find any evidence for absorption from molecular hydrogen. This X-shooter spectrum was previously also analysed by de Ugarte Postigo et al. (2018). 
J. Bolmer et al.: Evidence for diffuse molecular gas and dust in the hearts of gamma-ray burst host galaxies

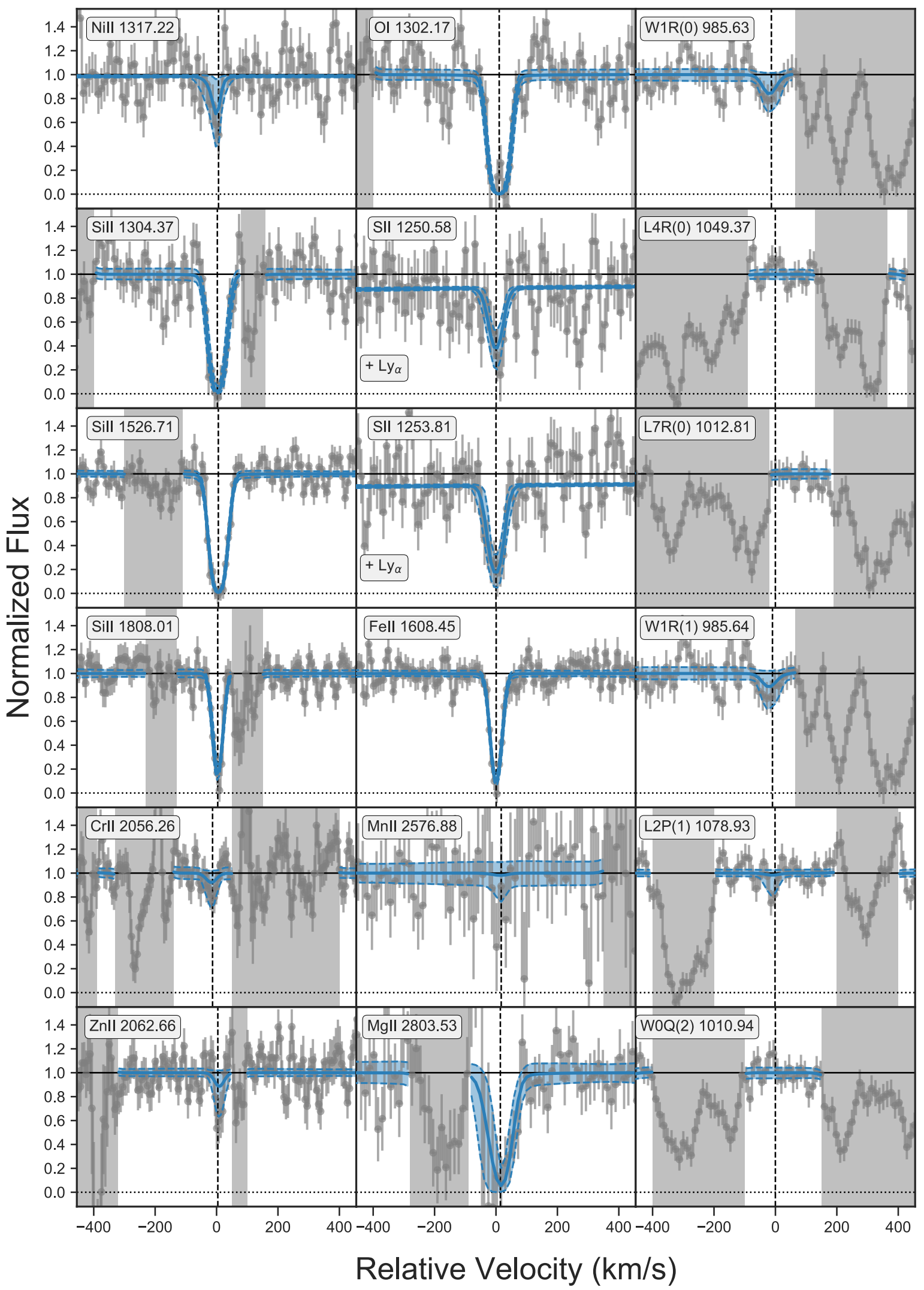

Fig. B.22. Results from fitting absorption lines in the X-shooter spectrum of GRB 170202A. As shown in the 6 subplots to the right, the spectrum is consistent with absorption from molecular hydrogen in the strongest $J=0$ and $J=1$ transitions at $\lambda \sim 985.6 \AA$, which leads us to claim a possible detection. 